\title{
Dissipation-induced instabilities in finite dimensions
}

\author{
R. Krechetnikov* and J. E. Marsden \\ California Institute of Technology, Pasadena, California 91125, USA
}

(Published 4 April 2007)

\begin{abstract}
The goal of this work is to introduce a coherent theory of the counterintuitive phenomena of dynamical destabilization under the action of dissipation. While the existence of one class of dissipation-induced instabilities was known to Sir Thomson (Lord Kelvin), it was not realized until recently that there is another major type of these phenomena hinted at by one of Merkin's theorems; in fact, these two cases exhaust all the generic possibilities. The theory grounded on the Thomson-Tait-Chetayev and Merkin theorems and on the geometric understanding introduced in this paper leads to the conclusion that ubiquitous dissipation is one of the paramount mechanisms by which instabilities develop in nature. Along with a historical review, the main theoretical achievements are put in a general context, thus unifying the current knowledge in this area and the multitude of relevant physical problems scattered over a vast literature. This general view also highlights the striking connection to various areas of mathematics. To appeal to the reader's intuition and experience, a large number of motivating examples are provided. The paper contains some new unpublished results and insights, and, finally, open questions are formulated to provide an impetus for future studies. While this review focuses on the finite-dimensional case, where the theory is relatively complete, a brief discussion of the current state of knowledge in the infinite-dimensional case, typified by partial differential equations, is also given.
\end{abstract}

DOI: $10.1103 /$ RevModPhys.79.519

PACS number(s): 45.20.Jj, 46.32.+x, 45.10.Na, 01.55.+b

\section{CONTENTS}

I. Introduction
A. Two key examples
B. Definition of dissipation-induced instability
C. Outline

II. Theoretical Framework
A. Euler-Lagrange equations and classification of forces
B. General linear formulation
C. On the notions of stability

III. Main Classical Results and Their Geometry
A. Thomson-Tait-Chetayev theory
1. Application 1: Radiation-induced instability
2. Application 2: The Levitron
3. A few more applications
B. Merkin theory
1. Application 1: Rotating shafts
2. Application 2: Secondary instability
C. On phase-space behavior
D. Summary and discussion
IV. Movements of Eigenvalues: Geometry in Spectral Space
A. Hamiltonian bifurcations
B. Dissipation-induced movements of eigenvalues
C. Connection to singularity theory
D. Summary
V. Dissipation-Induced Instabilities of Relative Equilibria
A. The concept of relative equilibria and the history of reduction
B. Cotangent bundle reduction

519
520
521
523
523
523
524
525
526
526
529
530
531
532
533
533
534
535

535

535

537

539

540

540

540

542

\footnotetext{
*Electronic address: rkrechet@cds.caltech.edu
}

C. Energy-momentum method 544

D. Summary 545

VI. Controlling Dissipation-Induced Instabilities $\quad 545$

A. Classical approach $\quad 545$

B. Geometric control 546

1. General methodology 546

2. Application to systems with symmetry 546

C. Remarks 547

VII. Towards an Infinite-Dimensional Theory 547

A. Baroclinic instability 547

B. General issues 548

C. On proving existence and stability 549

1. Stability 550

2. Existence $\quad 550$

D. Summary 551

VIII. Conclusions 551

References $\quad 551$

\section{INTRODUCTION}

The counterintuitive concept of a dissipation-induced instability, though coined just recently in the work of Bloch et al. (1994), takes its genesis from the classical Treatise on Natural Philosophy of Thomson and Tait (1879), whose results had been proved only in the 1950s by Chetayev (1961) and advanced by Merkin (1997). The growing number of physical examples and applications in the literature, some of which will be discussed in this work, demonstrates the need for a unified understanding of this apparently universal route to instabilities in various physical systems. The primary goal of this review is to introduce the reader to these classical results that, when seen from the right perspective, constitute a beautiful theory capable of explaining a variety of observed 


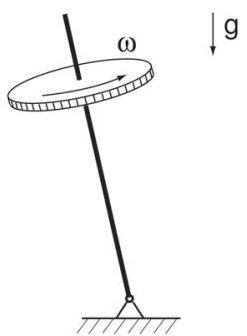

(a) Lagrange top

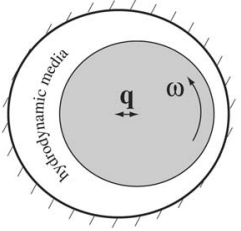

(b) Rotating shaft
FIG. 1. Two key examples.

instabilities in simple terms. At the same time, this theory has a nontrivial impact on the modern approach to mechanics including dynamical systems theory and geometry. Providing this link is another objective of our paper, which should also serve the purpose of introducing the reader to more recent results in this field as well as to many gaps in our understanding. We will try to balance clarity with technicalities to capture the interest of both the applied and more mathematically inclined readers. Therefore, we first formulate the main ideas in plain terms and then supply some technical discussion to engage the mathematical reader. In particular, on the abstract level (say, in the Hamiltonian setting) the subject of this paper is the study of the effect of nonconservative perturbations on the dynamics determined by the Hamiltonian, a map $H: T^{*} \mathrm{Q} \rightarrow \mathbb{R}$ from the phase space of a mechanical system, namely, a cotangent bundle $T^{*} \mathrm{Q}$, to a linear space, the real numbers $\mathbb{R}$. Often we will be considering the special case of mechanical systems in the presence of symmetries, which determine, in the absence of nonconservative perturbations, additional conserved quantities $\mathbf{J}$ taking values in a linear space of dimension $m$, so that the relevant object is the energy-momentum map $H \times \mathbf{J}: T^{*} \mathrm{Q} \rightarrow \mathbb{R} \times \mathbb{R}^{m}$. The nonconservative nature of perturbations is understood as resulting in dynamics whose time evolution satisfies $\mathrm{d}_{t} H \neq 0$ in general.

\section{A. Two key examples}

For the purpose of introducing the reader to the physical essence of the subject matter, we first discuss two key physical examples - the Lagrange top and the rotating shaft shown in Figs. 1(a) and 1(b), respectively-which will be analyzed further in Sec. II and used throughout the text. These two examples also serve the purpose of highlighting the Thomson-TaitChetayev and Merkin theories, and their synthesis suggests a general definition of dissipation-induced instability that we introduce below. While the formal statement of theorems will be given in Sec. II, here we state the corollaries of these theories in physical terms, which are well within the standard theoretical mechanics course, e.g., Goldstein (1956), relevant to our discussion. We start with a corollary of the Thomson-Tait-Chetayev theorem, which states that if a system with an unstable potential energy ${ }^{1}$ is stabilized with gyroscopic forces, then this stability is lost after the addition of arbitrarily small dissipation. The importance of this property in many physical and engineering applications should not be underestimated: the destabilizing effect of dissipation needs to be compensated in various gyroscopic devices by applying accelerating forces. To illustrate the above corollary of the Thomson-Tait-Chetayev theory and to appeal to the reader's intuition, we consider the following simple two degrees of freedom example.

Example (Lagrange top). The linearized dynamics of a Lagrange top [shown in Fig. 1(a)] has the form

$$
\begin{aligned}
& \ddot{q}_{1}+g \dot{q}_{2}-d \dot{q}_{1}+c_{1} q_{1}=0, \\
& \ddot{q}_{2}-g \dot{q}_{1}-d \dot{q}_{2}+c_{2} q_{2}=0,
\end{aligned}
$$

where $\mathbf{q}=\left(q_{1}, q_{2}\right)^{2}$ represents a linearized perturbation, and $g, d, c_{1}$, and $c_{2}$ are real constants. The origin of Eqs. (1.1) can be explained using the standard Euler angles $\alpha=q_{1}, \beta=q_{2}$, where $\dot{\phi}=\omega$ is the angular velocity of rotation around the axis of symmetry of the top in the first approximation. Then, in view of axisymmetry, the potentials are $c_{1}=c_{2}=-P l / J_{x}$ and the gyroscopic coefficient is $g=\omega J_{z} / J_{x}$, where $P$ is the gravitational force applied at the center of mass, located at distance $l$ from the point of support, and $J_{x}$ and $J_{z}$ are moments of inertia of the top in the trihedral $x y z$ coordinate system fixed to the top. For details, the reader may consult Merkin (1997). System (1.1) has an unstable equilibrium at the origin if $g=0$ and $c_{i}<0, i=1,2$, but can be stabilized by the addition of gyroscopic forces ${ }^{3}$ if $|g|>\sqrt{-c_{1}}+\sqrt{-c_{2}}$. As is easy to see directly by a spectral analysis, the addition of arbitrarily small dissipative forces, $d>0$, i.e., a symmetric term proportional to the velocity $\dot{\mathbf{q}}$, destabilizes the equilibrium.

The system (1.1) accounts for the dynamics of the perturbation $\mathbf{q}$, so that in this approximation the stability of the ordinary equilibrium $\mathbf{q}=\mathbf{0}$ can be ascertained. Note that the physical system actually has a relative equilibrium $^{4}$ (that is, the top is in steady rotation about its vertical axis), due to which a gyroscopic force appears in Eqs. (1.1). Therefore, the model (1.1) accounts for an instability of the top, which develops due to dissipative forces when the relative equilibrium is not maintained by an external source of energy. It is clear that dissipa-

\footnotetext{
${ }^{1}$ While the term "unstable potential energy" is intuitively transparent, its precise meaning will be clear from the subsequent discussion.

${ }^{2}$ In examples with a few degrees of freedom, we will be abusing the general index notation $q^{i}$ for configuration space coordinates to avoid double indices.

${ }^{3}$ In the stability analysis of two-dimensional linear systems, it is convenient to consider the complexified version in terms of $q_{1}+i q_{2}$.

${ }^{4}$ While the definition of relative equilibrium will be given in Sec. V, until then it can be understood informally as the situation in which the shape of the object under scrutiny does not change in time while the object as a whole is rotating.
} 
tion in this case leads to total energy decay (that is, the top slows down and eventually falls), as well as to the decrease of the energy of the perturbation $H=\frac{1}{2}\left[\dot{q}_{1}^{2}\right.$ $\left.+\dot{q}_{2}^{2}\right]+\frac{1}{2}\left[c_{1} q_{1}^{2}+c_{2} q_{2}^{2}\right]$ (that is, $\dot{H}<0$ ). This peculiar effect of dissipation is made possible, in this case, by an unstable potential energy, $c_{i}<0, i=1,2$ : while the perturbation grows, the sum $H$ of its kinetic and potential energies decreases.

As has been recently understood, the above situation that is accounted by the Thomson-Tait-Chetayev theory does not exhaust all the possibilities for the dissipationinduced instability phenomena (Krechetnikov and Marsden, 2006). Many studies in the earlier part of the 20th century demonstrated that dissipative forces, i.e., those that are proportional to velocities and having the form $D \dot{\mathbf{q}}$, with $D$ being a symmetric matrix, are not the only physically important ones of a nonconservative type (in the usual sense that the work done can be path dependent). Another physically significant and widespread class of nonconservative forces includes so-called positional forces, which are proportional to displacements and the associated matrices are skew symmetric. Follower forces, appearing, for example, in the problem of buckling, are a particular case of positional forces. Their theoretical basis was established by Merkin (1974, 1977), who proved a number of fundamental properties for the effects of these forces. To clarify the immediate and subsequent discussions, we just refer to one of them, which states that the introduction of nonconservative linear forces into a system with a stable potential and with equal frequencies destroys the stability regardless of the form of the nonlinear terms. The equal frequencies can come about, for instance, because of a system symmetry. To illustrate this theorem, consider the following example.

Example (rotating shaft). The system

$$
\begin{aligned}
& \ddot{q}_{1}+p q_{2}+c q_{1}=0, \\
& \ddot{q}_{2}-p q_{1}+c q_{2}=0,
\end{aligned}
$$

describes, among other systems, the linearized dynamics of a perturbation $\mathbf{z}$ of a rotating shaft (Kapitsa, 1939) shown in Fig. 1(b). While the origin of Eqs. (1.2) will be discussed in detail in Sec. III.B.1, we note that the positional force, i.e., the term $\pm p q_{i}$, is proportional to $\omega^{2}$, where $\omega$ is the rotation rate of the shaft as indicated in Fig. 1(b). The corresponding characteristic equation shows that the addition of nonzero nonconservative positional forces (that is, $p \neq 0$ ) to a system with a stable potential energy makes it unstable. The origin of the skew-symmetric positional forces lies in the friction between the rotating shaft and the hydrodynamic media that fills the space between shell and shaft, and in the asymmetry of the gap when the shaft is displaced from the axis of symmetry (Kapitsa, 1939).

By analogy to example (1.1), system (1.2) accounts for the evolution of a perturbation $\mathbf{q}$, which measures the departure from equilibrium. The equilibrium corresponds to a relative equilibrium of a rotating shaft, i.e., a uniformly rotating state with angular velocity $\omega$, due to which the positional forces appear, as will be seen in Sec. III.B.1. It is notable that, in contrast to Eqs. (1.1), the energy of the disturbance eventually grows. Despite this, the positional forces in this case are dissipative, since the total energy of the physical system decays under their action, i.e., if one stops maintaining the relative equilibria by discontinuing the application of an external source of energy, the shaft will stop rotating. However, it should be kept in mind that not all positional forces are dissipative, as can be seen in the example of an instability of an elastic bar with a follower force (Nikolai, 1939) even though the linearized dynamics of perturbation is given by the same equations (1.2). This differentiation of positional forces into dissipative and nondissipative depends on whether or not the particular physical system is closed or open. Concluding the discussion of (1.2) we note that, in contrast to the case (1.1), the model (1.2) accounts for an instability of the rotating shaft whether the relative equilibrium is maintained or not.

\section{B. Definition of dissipation-induced instability}

The common features of the above two examples are that the instability develops due to withdrawal of energy from the basic state (that is, the relative equilibrium) and the total energy of the whole physical system would decay, if the relative equilibrium is not maintained, while the energy of the perturbation may grow. Motivated by all these physical considerations, we introduce the following physical definition of dissipative forces.

Definition 1. A set of nonconservative forces acting on a mechanical system with a relative equilibrium is called dissipative if under the action of these forces and in the absence of the forces that work against these nonconservative forces in order to maintain the (relative) equilibrium, the total mechanical energy of the whole physical system decreases.

This allows us to define a generalized notion of dissipation-induced instability:

Definition 2. A conservative system with a spectrally stable (relative) equilibrium is said to suffer from dissipation-induced instability if the introduction of dissipative forces destabilizes this equilibrium in the Lyapunov sense.

From a physical standpoint, dissipation-induced instabilities are interesting when definition 2 is used in a stronger form, namely, when stability of a conservative system holds not only in the spectral sense, but also in the Lyapunov sense, which would correspond to a strong version of definition 2. There are a number of known examples of this kind in finite dimensions, and we have been able to establish this type of dissipationinduced instability in an infinite-dimensional example, namely, the baroclinic instability (see Sec. VII for further discussion).

Now we can reconcile the preceding discussion of dissipation-induced instabilities with the first law of thermodynamics. Figure 2 depicts a hierarchy of mathematical descriptions of the same physical phenomena, but with different degrees of detail, which are related to the 


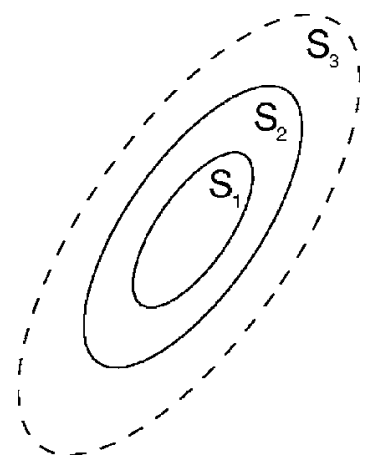

FIG. 2. The definition of dissipation-induced instabilities.

interaction of a physical system with surrounding systems. Assume that we have a description of the Lagrange top as an isolated (closed) system at the level $S_{2}$, which includes the full dynamical description of the rigid body and its interaction with a supporting point and air through friction (say, we place the experimental setup-Lagrange top spinning on a substrate-in a thermostat, which isolates the system from the ambient environment). If $H$ stands for the mechanical energy, and $Q$ stands for all other nonmechanical contributions to the energy (chemical, thermal, etc.), then the total energy $U=H+Q$ is conserved based on our assumption that the system is isolated. We have distinguished the mechanical energy here, since for the present discussion we focus on instability in a mechanical sense. Next, since we are concerned with the stability question, we can decompose the dynamics into a basic state, which is the relative equilibrium in this case, and a perturbation, which is a departure of the dynamics from the basic state. Therefore, the energy evolution obeys

$$
\begin{aligned}
& S_{2}: \quad \mathrm{d} U_{2}=0, \quad \mathrm{~d} Q_{2}>0, \quad \mathrm{~d} H_{2}^{\text {total }}<0 \\
& \text { with } \mathrm{d} H_{2}^{\text {perturb. }}<0, \quad \mathrm{~d} H_{2}^{\text {basic state }}<0,
\end{aligned}
$$

where all the indexes are self-explanatory and the total mechanical energy is a sum of the mechanical energies of perturbation and basic state $H_{2}^{\text {total }}=H_{2}^{\text {perturb. }}$ $+H_{2}^{\text {basic state }}$ with $H_{2}^{\text {basic state }}$ determined by the dynamics in the absence of perturbation and $H_{2}^{\text {perturb. }}=H_{2}^{\text {total }}$ $-H_{2}^{\text {basic state }}$. Now one can restrict the consideration to the subsystem $S_{1} \subset S_{2}$, which is, obviously, not closed, and which accounts for the evolution of a disturbance only. Naturally, in this case we get $S_{1}: \mathrm{d} H_{1}^{\text {total }}$ $=\mathrm{d} H_{1}^{\text {perturb }}<0$. On the other hand, in the case of a rotat ing shaft at the analogous level of description $S_{2}$, when the system is considered closed, we get

$$
\begin{aligned}
& S_{2}: \quad \mathrm{d} U_{2}=0, \quad \mathrm{~d} Q_{2}>0, \quad \mathrm{~d} H_{2}^{\text {total }}<0 \\
& \text { with } \mathrm{d} H_{2}^{\text {perturb. }}>0, \quad \mathrm{~d} H_{2}^{\text {basic state }}<0,
\end{aligned}
$$

where one can notice a change in the sign of $\mathrm{d} H_{2}^{\text {perturb. }}$ compared to the Lagrange top case (1.3), since the energy of the disturbance grows. The above two key cases-Lagrange top and rotating shaft—evidently span

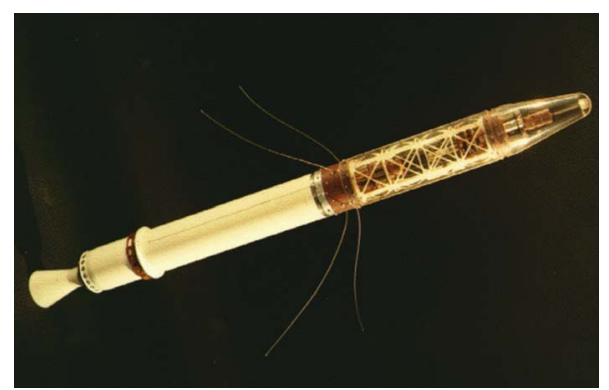

FIG. 3. (Color online) Explorer I (courtesy of JPL, NASA).

all the possibilities. The picture does not change even if we refine the description by going to the next level, $S_{3}$, and so on. At this stage, note that we have at our disposal only two categories of systems, namely, Hamiltonian, $\mathrm{d} H=0$, and dissipative, $\mathrm{d} H<0$. Therefore, all instabilities can be divided into two classes: (i) those that are accounted for by the Hamiltonian description, and (ii) those that are due to dissipation. Since our vocabulary contains only two words, namely, "Hamiltonian" and "dissipative," there are no other options for the natural occurrence of instabilities. Thus, the instabilities are intrinsically either Hamiltonian or dissipation induced.

Having the above clarification of the physical meaning and occurrence of dissipation-induced instabilities, it is worth mentioning the distinction between our definition 2 and the term dissipative instability used, for example, by Casti et al. (1998) in the problem of gravitational instability of interpenetrating galaxies. Two interpenetrating galaxies always experience Jeans instability, i.e, there is always a band of wave numbers $k$ in which a linear perturbation $e^{i(\omega t-k x)}$ of frequency $\operatorname{Re}(\omega)$ grows with the rate $-\operatorname{Im}(\omega)$. This is analogous to the case of two stationary galaxies, which are always unstable since the dispersion relation is $\omega^{2}=k^{2}-1$ and thus there is no bifurcation parameter controlling the transition from the stable to the unstable case. An introduction of dissipation, which may be due to collisions, simply increases the band of unstable wave numbers, and thus suggests the term dissipative instability (Casti et al., 1998), which probably should be named dissipation-enhanced instability. Thus, the system is unstable in both the conservative and dissipative cases and therefore is not a system with a dissipation-induced instability in our sense.

Concluding this introduction, we could not resist mentioning one of the famous examples-Explorer I shown in Fig. 3-of dissipation-induced instabilities, the lack of theoretical understanding of which has led to a technological failure. Explorer I, launched in January 1958 shortly after Russian Sputniks were launched in October and November 1957, was long and narrow like a pencil. It was supposed to rotate around its own centerline (which is the axis of minimum moment of inertia), but definitely was not supposed to rotate end over end (like a windmill blade), which would correspond to the axis 
with a maximum moment of inertia. However, once $E x$ plorer I made just one Earth orbit, it flipped over and from then on it windmilled. This instability was caused by a flexing of its antennae, which dissipated a small amount of rotational energy. The amazing part of this story is that Stanford University astronomer Ronald Bracewell tracked the first Sputnik and determined that it was spinning in its maximum moment of inertia mode, which was also consistent with the way galaxies behave. However, security concerns of the Jet Propulsion Laboratory did not let him to talk to engineers and the $E x$ plorer I was launched as is. The warning of Bracewell appeared in the open literature (Bracewell and Garriot, 1958) only seven months after the launch.

\section{Outline}

Having introduced the reader to the subject matter from intuitive and historical prospectives, we proceed with the presentation of the paper as follows. Section II deals with the basic theoretical framework; the reader is introduced to the Hamiltonian and Lagrangian setup in the context of classical mechanics in Sec. II.B, other basic concepts such as the classification of forces in Sec. II.A, and various stability notions in Sec. II.C. Section III is devoted to the classical results in this field, namely, those due to Thomson, Tait, and Chetayev in Sec. III.A and due to Merkin in Sec. III.B. Based on these results, we develop a geometrical understanding of dissipationinduced instabilities in phase space through the notions of the second variation $\delta^{2} H$ of the Hamiltonian $H$ and the elementary phase-space volume behavior in Sec. III.C. In the conclusion of Sec. III.D, we introduce the most fundamental classification of dissipation-induced instabilities motivated by geometrical considerations. Both the Thomson-Tait-Chetayev and Merkin theories in Sec. III are illustrated with many physical examples. In Sec. IV, we discuss the manifestation of dissipationinduced instabilities in a spectral space. After reminding the reader of the classical results on Hamiltonian bifurcations in Sec. IV.A, we proceed with the discussion of the movement of eigenvalues due to dissipative effects in Sec. IV.B, and the connection to singularity theory in Sec. IV.C.

While the presentation up to this point has been on dissipation-induced instabilities of ordinary equilibria, in Sec. V the case of relative equilibria is discussed. At this point, we introduce the geometric concepts necessary for the discussion of relative equilibria in Sec. V, and control of dissipation-induced instabilities in Sec. VI. We begin Sec. V by introducing the concept of relative equilibria in Sec. V.A, and then discuss the methodology of dealing with relative equilibria through the reduction procedure in Sec. V.B and the energymomentum method in Sec. V.C. In view of the importance of controlling dissipation-induced instabilities in various engineering applications, we devote Sec. VI to this subject, and address both the classical and geometric approaches in Secs. VI.A and VI.B, respectively. The paper concludes in Sec. VII with a discussion of our re- cent understanding of dissipation-induced instabilities in infinite-dimensional systems, as well as the most troublesome issues, such as the function-theoretical questions involving the compatibility of existence and stability in Secs. VII.B and VII.C. The central physical example in this section is the baroclinic instability, discussed in Sec. VII.A.

\section{THEORETICAL FRAMEWORK}

The examples of the Lagrange top and rotating shaft discussed in the Introduction were analyzed using the linearizations (1.1) and (1.2), respectively. In this section, we present a system in a general form, to which the Thomson-Tait-Chetayev and Merkin theories are applicable. Though these theories apply to many other situations (such as nonholonomic systems, etc.), for illustration and for the purpose of introducing the necessary definitions, we appeal to the classical way of arriving at the general formulation from first principles.

\section{A. Euler-Lagrange equations and classification of forces}

The mathematical formulation we consider throughout this paper will be the linearization of the EulerLagrange equations for a Lagrangian $L$ with generalized forces $\tilde{Q}_{i}$,

$$
\frac{\mathrm{d}}{\mathrm{d} t} \frac{\partial L}{\partial \dot{q}^{i}}-\frac{\partial L}{\partial q^{i}}=\tilde{Q}_{i},
$$

the classical mechanics derivation of which is provided below in the context of a system of particles for the reader's convenience.

Classical derivation. Consider a mechanical system of $N$ particles of masses $m_{\nu}$ and with positions given by vectors $r_{\nu}$ in $\mathbb{R}^{3}$, for example, under the action of active forces $F_{\nu}$ and of $d$ geometric constraints $f_{\alpha}\left(t, r^{v}\right)=0, \nu$ $=1, \ldots, N, \alpha=1, \ldots, d$. The latter implies that the system under consideration is holonomic, since there are no nonintegrable kinematic constraints $f\left(t, r^{v}, \dot{r}^{\nu}\right)=0, \quad \nu$ $=1, \ldots, N$ imposed. Because of the presence of the constraints, there are reaction forces $R_{\nu}$ so that Newton's second law reads $m_{\nu} w_{\nu}=F_{\nu}+R_{\nu}$, where $w_{\nu}$ are accelerations. In the case of ideal constraints, i.e., when the work $R_{\nu} \delta r^{\nu}$ (Einstein summation rule is assumed) on virtual displacements $\delta r_{\nu}$ vanishes, we arrive at the D'Alembert principle

$$
\left(F_{\nu}-m_{\nu} w_{\nu}\right) \delta r^{\nu}=0
$$

In view of the constraints on the dynamics, the number of independent degrees of freedom is $3 N-d=n$ (for three-dimensional physical space), and therefore one can introduce $n$ independent generalized coordinates $q^{1}, \ldots, q^{n}$, by making a transformation $r^{\nu}$ $=r^{\nu}\left(t, q^{1}, \ldots, q^{n}\right)$. As a result, Eq. (2.2) can be simplified since the work of active forces is $\delta A=F_{\nu} \delta r^{\nu}=Q_{i} \delta q^{i}$, where $Q_{i}=F_{\nu} \partial r^{\nu} / \partial q^{i}$ are generalized forces, while the work of the inertia forces is $\delta A_{I}=-m_{\nu} w_{\nu} \delta r^{\nu}=-Z_{i} \delta q^{i}, Z_{i}$ 
$=(\mathrm{d} / \mathrm{d} t)\left(\partial T / \partial \dot{q}^{i}\right)-\partial T / \partial q^{i}$, where $T=\frac{1}{2} m_{\nu} \dot{r}^{\nu 2}$ is the kinetic energy of the system. Combining the last two equations with Eq. (2.2) yields the Lagrangian equations of the second kind,

$$
\frac{\mathrm{d}}{\mathrm{d} t} \frac{\partial T}{\partial \dot{q}^{i}}-\frac{\partial T}{\partial q^{i}}=Q_{i}
$$

since the variations $\delta q^{i}$ are independent. Concluding, Eq. (2.3) is valid for holonomic systems with ideal constraints. Further we restrict ourselves to holonomic systems with stationary constraints, i.e., scleronomic versus rheonomic (with time-dependent constraints). In this case, the kinetic energy expression simplifies to the quadratic form $T=\frac{1}{2} a_{i k} \dot{q}^{i} \dot{q}^{k}$ with $\operatorname{det} a_{i k} \neq 0$. Decomposing further the generalized forces $Q_{i}$, which can be nonlinear, into potential and nonpotential parts $Q_{i}=-\partial \Pi / \partial q^{i}$ $+\tilde{Q}_{i}$, we arrive at the Euler-Lagrange equations (2.1).

Based on the energy $E=T+\Pi$ (kinetic plus potential) and the rate of change of energy equation $(\mathrm{d} / \mathrm{d} t) E$ $=\tilde{Q}_{i} \dot{q}^{i}$, Thomson and Tait (1879) further classify the nonpotential forces $\tilde{Q}_{i}$ into gyroscopic, $\tilde{Q}_{i} \dot{q}^{i}=0$, dissipative, $\tilde{Q}_{i} \dot{q}^{i} \leqslant 0$, and accelerating, $\tilde{Q}_{i} \dot{q}^{i} \geqslant 0$. The dissipative forces, after Chetayev (1961), are distinguished into complete and partial dissipation if the power $\tilde{Q}_{i} \dot{q}^{i}$ is negative definite or simply definite, respectively.

While the above definitions are generally valid for nonlinear forces, the most familiar definitions correspond to the linear case, where further clarification is possible. Linear gyroscopic forces have a skewsymmetric structure: $\tilde{Q}_{i}=\gamma_{i k} \dot{q}^{k}$, where $\gamma_{i k}=-\gamma_{k i}$, versus dissipative forces, which have a symmetric form: $\tilde{Q}_{i}$ $=-b_{i k} \dot{q}^{k}$, where $b_{i k}=b_{k i}$ and thus can also be expressed in terms of the Rayleigh dissipative function $R$ $=\frac{1}{2} b_{i k} \dot{q}^{i} \dot{q}^{k} \quad$ as $\quad \tilde{Q}_{i}=\left(\partial / \partial \dot{q}^{i}\right) R$. In accordance with Chetayev's definition, if $R$ is a positive definite quadratic form, then the dissipation is complete. Physically, these forces are due to the motion in a resisting medium, etc., when the resistance depends only on the speed of motion. Special kinds of forces, not evident from Thomson and Chetayev's classification, are so-called nonconservative positional forces, which change the energy of the system, but depend on the coordinates only: $\tilde{Q}_{i}=-p_{i k} q^{k}$, where $p_{i k}=-p_{k i}$. These forces are also called circular (Ziegler 1953), pseudogyroscopic, forces of radial correction or limited damping, and are more common than is usually supposed (Ziegler, 1953). Physically, positional forces occur in elastic systems subject to the forces whose line of action is always tangential to the elastic axis (Nikolai, 1939; Herrman, 1967; Langthjem and Sugiyama, 2000), in the motion of elastic bodies in a viscous medium (Bolotin, 1963), rotor instability in a hydrodynamic medium (Kapitsa, 1939), and many other systems. This classification of linear forces is ultimately important in studying the linear stability of various systems and allows one to identify the nature of various terms in the linearized dynamics and directly apply the theoretical results of the next section.

While the classical definitions by Thomson and Tait (1879) and Chetayev (1961) cover the case of general nonlinear gyroscopic, dissipative, and accelerating forces, they do not reveal the definition of general nonconservative positional forces. However, as suggested by Merkin (1974), the property of orthogonality of a positional force $\tilde{Q}_{i}$ and the radius vector $\mathbf{q}$ in the linear case, namely, $p_{i k} q^{k} q^{i}=0,{ }^{5}$ can be extended to the nonlinear case, namely, i.e., $\tilde{Q}_{i} q^{i}=0$, in order to define the nonlinear nonconservative positional forces $\tilde{Q}_{i}$. While the general classification of the physical nature of forces $\tilde{Q}_{i}(\mathbf{q}, \dot{\mathbf{q}})$, in the case of general dependence on positions $\mathbf{q}$ and velocities $\dot{\mathbf{q}}$, is not available, the decomposability of particular dependencies, $\tilde{Q}_{i}(\mathbf{q})$ or $\tilde{Q}_{i}(\dot{\mathbf{q}})$, into physically meaningful skew and symmetric components has been explored by Merkin (1974).

\section{B. General linear formulation}

Decomposition of the Lagrange equations (2.1) into their linearization at the equilibrium, and into the remaining nonlinear terms, yields

$$
M \ddot{\mathbf{q}}+\underset{\text { gyroscopic }}{S \dot{\mathbf{q}}}+\underset{\text { dissip. }}{D \dot{\mathbf{q}}}+\underset{\text { potential }}{C \mathbf{q}}+\underset{\text { noncons. }}{P \mathbf{q}}=\mathcal{N},
$$

where $M, D, C$ are symmetric, while $S, P$ are skewsymmetric matrices, and $\mathcal{N}$ is a nonlinear part. This formulation can be simplified using the classical matrix analysis theorem, which states that if the square matrices $M$ and $C$ are both symmetric and $M$ is also sign definite, then there exists a nonsingular matrix $\Lambda$ such that $\Lambda^{T} M \Lambda=I, \Lambda^{T} C \Lambda=C_{0}$. Here $I$ is the identity matrix and $C_{0}$ is a diagonal matrix with each element of diag $C_{0}$ $=\left\{c_{1}, \ldots, c_{n}\right\}$ called a stability coefficient following Poincaré. This is a particular case of the standard technique for diagonalization of pencils of matrices or quadratic forms (Gantmacher, 1977). This linear algebra theorem implies, in particular, that there exists a linear change of variables $\mathbf{q} \rightarrow \boldsymbol{\theta}$ such that $T=\frac{1}{2} a_{i k} \dot{q}^{i} \dot{q}^{k}$ simplifies to $T=\frac{1}{2} \Sigma_{i}\left(\dot{\theta}^{i}\right)^{2}$ and $\Pi=\frac{1}{2} c_{i k} q^{i} q^{k}$ reduces to $\Pi=\frac{1}{2} \Sigma_{i} \lambda_{i}\left(\theta^{i}\right)^{2}$, with $\lambda_{i}$ being the stability coefficients. The resulting system in new variables is $\ddot{\theta}^{i}+\lambda_{i} \theta^{i}=$ nonlinear terms. The number of negative $\lambda_{i}$ 's is called the degree of instability. Applying this theorem to our system (2.4), i.e., $\mathbf{q} \rightarrow \Lambda \mathbf{q}$, we end up with a simpler version of Eq. (2.4), where $M=\mathrm{id}$, matrix $C \equiv C_{0}$ is diagonal now with no zero elements and we keep the original notations for the other matrices, and under the performed transformation they retain their symmetry and skew-symmetry properties. Systems of the form (2.4) are sometimes called Chetayev systems (Bloch et al., 1994, 2004) and, as we will see in Sec. V, they also represent a normal form for a simple

\footnotetext{
${ }^{5}$ This holds because of the skew symmetry of $p_{i k}$.
} 
mechanical system ${ }^{6}$ linearized about a relative equilibrium modulo an Abelian symmetry group.

Even though the system (2.4) is nonconservative, with this definition of the Hamiltonian,

$$
H=\frac{1}{2} \mathbf{p}^{T} \mathbf{p}+\frac{1}{2} \mathbf{q}^{T} C_{0} \mathbf{q}, \quad \mathbf{p}=\dot{\mathbf{q}},
$$

the system can be recast into a symplectic-metriplectic form. With $\mathbf{z}=(\mathbf{q}, \mathbf{p})$, Eq. (2.4) can be written as

$$
\dot{\mathbf{z}}=(\mathbb{J}+\mathbb{G}) H_{\mathbf{z}},
$$

where the operators $J$ and $G$ are given by

$$
J=\left[\begin{array}{cc}
0 & \text { id } \\
-\mathrm{id} & -S
\end{array}\right], \quad G=\left[\begin{array}{cc}
0 & 0 \\
-P C_{0}^{-1} & -D
\end{array}\right],
$$

where the matrix $J$ is skew symmetric and is called a Poisson operator, while the matrix $G$ in the absence of nonconservative positional forces is symmetric and called a metriplectic operator in view of its similarity to a metric tensor (Morrison, 1986). In the presence of nonconservative positional forces, the matrix $\mathrm{G}$ need be neither symmetric nor skew symmetric. One can regard J and $\mathrm{G}$ as determining the geometry of phase space. The operator $J$ comes from symplectic geometry and (i) is nonsingular, (ii) is skew symmetric, and (iii) obeys the Jacobi relation.

Consider the case when Eq. (2.6) is a canonical Hamiltonian system, i.e.,

$$
\dot{\mathbf{z}}=J H_{\mathbf{z}}, \quad J^{T}=-J, \quad J=\left[\begin{array}{cc}
0 & \mathrm{id} \\
-\mathrm{id} & 0
\end{array}\right] .
$$

Since $H(\mathbf{z})=\frac{1}{2} \mathbf{z}^{T} \mathcal{A} \mathbf{z}$ with $\mathcal{A}^{T}=\mathcal{A}$, as follows from Eq. (2.5), where $\mathcal{A}$ can be time dependent, the system (2.8) may be written $\dot{\mathbf{z}}=A \mathbf{z}$, where $A=J \mathcal{A}$. Because the system is Hamiltonian, the initial condition $\mathbf{z}_{0}$ is transformed into a solution $\mathbf{z}(t)$ by a symplectic map $\mathbf{z}(t)=\Phi\left(t, t_{0}\right) \mathbf{z}_{0}$, i.e., by the state transition matrix $\Phi\left(t, t_{0}\right)$, which is a solution of $\dot{\Phi}=A \Phi$. Hence, if the solution is stable, then all eigenvalues of $\Phi$ lie on the unit circle. We recall that because $\Phi$ is symplectic, if $\lambda$ is an eigenvalue of $\Phi$, then so are $1 / \lambda, \bar{\lambda}, 1 / \bar{\lambda}$, i.e., all eigenvalues are symmetric with respect to the real axis and unit circle (PoincaréLyapunov lemma). It is easy to prove (Arnold, 1978) that for $\Phi$ to be stable, it is sufficient that all eigenvalues lie on a unit circle and are simple. Therefore, the necessary condition (but not sufficient) for instability to occur is a collision of eigenvalues on a unit circle. To isolate the conditions when the collision does not provoke instabil-

\footnotetext{
${ }^{6}$ We distinguish between simple mechanical systems, for which the Hamiltonian is separable $H=T+\Pi$, and natural mechanical systems, when it might be nonseparable, e.g, when terms of a gyroscopic type are present. In the text we consider both types depending on the context. Note that Arnold (1978, 1993) adopted a different definition for natural (Lagrangian) mechanical systems, namely, $L=T-\Pi$ with $T$ being $\frac{1}{2}\langle\dot{\mathbf{q}}, \dot{\mathbf{q}}\rangle$, where $\langle\cdot, \cdot\rangle$ is the Riemannian metric.
}

ity (Daleckii and Krein, 1974), the definition of an eigenvalue sign is needed. An eigenvalue $\lambda$, such that $|\lambda|=1$, $\lambda^{2} \neq 1$, is called positive (negative) if $[\Phi \xi, \xi]>0(<0)$ for every eigenvector $\xi$ of the real $\Phi$-invariant plane $\sigma$ corresponding to the eigenvalues $\lambda$ and $\bar{\lambda}$. According to this definition, collision of two eigenvalues with identical signs on the unit circle does not provoke instability (Arnold and Avez, 1968; Daleckii and Krein, 1974).

When studying linear stability, we deal with the linearization operator $L=J D_{\mathbf{z}}^{2} H(0)$, which is infinitesimally symplectic. This has the consequence that if $\lambda$ is an eigenvalue of $L$, then so is $-\lambda$. Therefore, a necessary condition for the equilibrium to be stable is that $\operatorname{Re} \operatorname{spec}(L)=0$.

Note that Eq. (2.8) remains Hamiltonian even if gyroscopic forces are added: the effect of the gyroscopic forces can be represented via a noncanonical Poisson bracket, in which a sum on repeated indices is understood,

$$
\{F, H\}=F_{q} H_{p_{i}}-F_{p_{i}} H_{q^{i}}-S_{i j} F_{p_{i}} H_{p_{j}} .
$$

Gyroscopic forces can provide an exchange of energy among the modes, and thus can significantly alter the behavior of the system; for example, they can stabilize an equilibrium with a nonzero degree of instability.

The fundamental classical stability theorems (Thomson and Tait, 1879; Chetayev, 1961; Merkin, 1997), some of which will be discussed in this review, can be deduced either by appealing to spectral properties of the dynamical system (2.4) or by appealing to the geometrical properties of Eq. (2.6). In particular, a linear stability analysis of Eq. (2.6) amounts to the eigenvalue analysis of $(\mathrm{J}$ $+G) H_{\mathbf{z z}}$. In view of the simplicity of the symplectic operator in the Hamiltonian case, the stability of the system naturally can be inferred from the second variation of the Hamiltonian, that is, the Hessian matrix of the second derivatives $H_{z z}$, though in a nontrivial way as discussed in the next subsection and in Sec. V.C.

\section{On the notions of stability}

To conclude this section, we discuss various notions of (in)stability used throughout the paper. In the general discussion, we refer to the system (2.6) and in the stability analysis to an equilibrium point $\mathbf{z}_{e}$ of that system, which satisfies $(J+G)(\partial H / \partial \mathbf{z})\left(\mathbf{z}_{e}\right)=0$. The nonlinear (Lyapunov) stability of $\mathbf{z}_{e}$ is defined as follows.

Definition 3. The equilibrium point $\mathbf{z}_{e}$ is Lyapunov stable if, for all $\epsilon>0$, there is a $\delta(\epsilon)>0$ such that if

$$
\left\|\mathbf{z}(0)-\mathbf{z}_{e}\right\|<\delta,
$$

then

$$
\left\|\mathbf{z}(t)-\mathbf{z}_{e}\right\|<\epsilon,
$$

for all $t>0$. If $\delta$ can also be chosen such that if $\| \mathbf{z}(0)$ $-\mathbf{z}_{e} \|<\delta$, then $\lim _{t \rightarrow \infty} \mathbf{z}(t)=\mathbf{z}_{e}$, the equilibrium is called asymptotically stable.

If Eq. (2.6) is linear, then stability is referred to as linear stability. If the stability of a linear system with the 
operator $(J+G) \partial H / \partial \mathbf{z}$ is investigated through its spectral properties, i.e., by analyzing the eigenmodes $\mathbf{z}=\hat{\mathbf{z}} e^{\lambda t}$, then the stability characteristics are understood according to the notion of spectral stability: the origin is spectrally stable if there are no eigenvalues $\lambda$ with $\operatorname{Re} \lambda>0$. While for practical purposes the most relevant definition is the Lyapunov one, which guarantees nonlinear stability, in reality one often ends up demonstrating weaker versions of stability, namely, linear and spectral stability. Therefore, it is very important to appreciate their interrelation, at least with the help of examples. First of all, the linear and nonlinear stability definitions do not imply each other: the system with potential $V(q)=q^{4} / 4$ demonstrates nonlinear stability, but linearization around the origin, $\dot{p}=0$ and $\dot{q}=p$, produces a solution growing linearly in time, i.e., it is linearly unstable. However, this example is spectrally stable; thus, spectral stability does not imply even linear stability, however the converse is true. Another example by Cherry (1925), given below in a different context, proves that linear stability does not imply nonlinear stability. A similar example has been discussed by Pollard (1966) and Siegel and Moser (1971). Before going into further discussion, we need the classical stability theorems in the conservative case. The oldest result goes back to Lagrange (1788).

Theorem 1 (Lagrange, 1788). If the Hamiltonian is separable, i.e., $H=\frac{1}{2} \mathbf{p}^{T} I \mathbf{p}+V(\mathbf{q})$, and $\mathbf{q}_{e}$ is a local strict minimum of $V(\mathbf{q})$, then the equilibrium point $\left(\mathbf{p}_{e}=\mathbf{0}, \mathbf{q}_{e}\right)$ is stable.

A converse is not true, as demonstrated by the following example due to Wintner (1947). Consider the $C^{\infty}$ potential

$$
V(q)= \begin{cases}e^{-q^{-2} \cos q^{-1}}, & q \neq 0 \\ 0, & q=0,\end{cases}
$$

from which it follows that the equilibrium $q_{e}=0$ is stable, but the origin is not a local minimum in view of wild oscillations. Despite this example, additional conditions [apart from the absence of a minimum of $V(\mathbf{q})$ ] allow one to formulate the converse to the Lagrange-Dirichlet theorem, cf. Rumyantsev and Sosnitskii (1994), and references therein. The Lagrange theorem was proved by Dirichlet (1846) based on the definition of nonlinear stability and with the help of level sets of the energy functional, which imposes restrictions on the behavior of trajectories in phase space. These pure geometric considerations served as the impetus for Lyapunov's direct method (based on Lyapunov functions) and also has lead to the generalization of the Lagrange theorem in which the Hamiltonian is not separable. The latter major result is now known as the Lagrange-Dirichlet theorem.

Theorem 2 (Dirichlet, 1846). If the second variation (Hessian) of the Hamiltonian, i.e., $H_{\mathbf{z z}}$ with $\mathbf{z}=(\mathbf{q}, \mathbf{p})$, is definite at the equilibrium point $\mathbf{z}_{e}$, then the equilibrium point is stable.

In the separable case it is easy to establish the connection between definiteness of the second variation of $H(\mathbf{q}, \mathbf{p})$ and the existence of a local minimum of $V(\mathbf{q})$, as

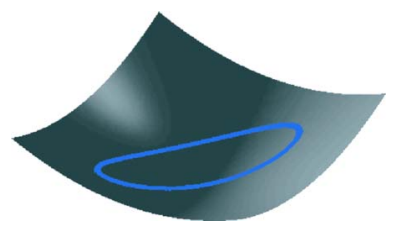

FIG. 4. (Color online) System with stable potential.

depicted in Figs. 4 and 5. As an illustration of the Lagrange-Dirichlet principle, we refer to Fig. 4, where a trajectory of a stable two-dimensional system

$$
\begin{aligned}
& \ddot{q}_{1}+c_{1} q_{1}=0, \\
& \ddot{q}_{2}+c_{2} q_{2}=0,
\end{aligned}
$$

with $c_{i}>0$, is projected onto the potential energy surface $V\left(q_{1}, q_{2}\right)=\frac{1}{2}\left(c_{1} q_{1}^{2}+c_{2} q_{2}^{2}\right)$. It should be noted that the Dirichlet theorem is not necessary, as illustrated by the linear part of the example due to Cherry (1925). Consider the Hamiltonian of two coupled oscillators

$$
H=\frac{1}{2} \omega_{2}\left(p_{2}^{2}+q_{2}^{2}\right)-\frac{1}{2} \omega_{1}\left(p_{1}^{2}+q_{1}^{2}\right),
$$

from which one can observe that the Hamiltonian produces two stable oscillators, but its second variation is indefinite.

\section{MAIN CLASSICAL RESULTS AND THEIR GEOMETRY}

\section{A. Thomson-Tait-Chetayev theory}

Here we discuss two of the theorems by Thomson, Tait, and Chetaev, which are directly pertinent to dissipation-induced instabilities. Instability in this section is understood in the Lyapunov sense; however, in some instances we prove spectral instability, which im-

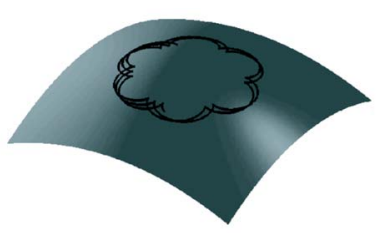

(a) Gyroscopic stabilization: even degree of instability

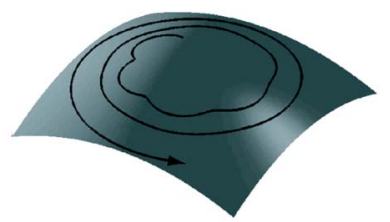

(c) Dissipative destabilization of gyroscopically stable system

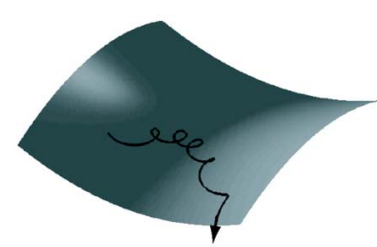

(b) Gyroscopic failure: odd degree of instability

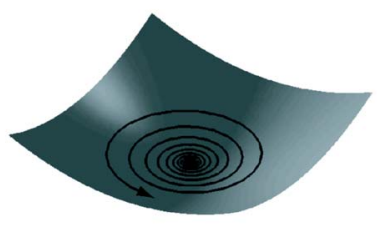

(d) Nonconservative positional destabilization of potentially stable system
FIG. 5. (Color online) Projection of dynamics onto the potential energy surface. 
plies (and gives sharper results than) Lyapunov instability and will be the subject of further discussion in Sec. IV.B.

Theorem 3 (Thomson-Tait-Chetayev). If the system $\ddot{\mathbf{q}}$ $+C_{0} \mathbf{q}=0$ has a nonzero degree of instability, then the equilibrium remains unstable after the addition of gyroscopic and dissipative forces with complete dissipation (that is, $\tilde{Q}_{i} \dot{q}^{i}$ is negative definite).

Proof. The presence of a nonzero degree of instability implies that the potential energy $\Pi$ will assume negative values in the vicinity of an equilibrium point. The energy balance,

$$
\frac{\mathrm{d} E}{\mathrm{~d} t}=\tilde{Q}_{i} \dot{q}^{i}, \quad E=T+\Pi,
$$

where $\tilde{Q}_{i}$ are dissipative forces with complete dissipation, implies that we can use the function $V=-E$ on the manifold $K(\mathbf{q} \neq \mathbf{0}, \dot{\mathbf{q}}=\mathbf{0})$ in Krasovsky's theorem of instability $^{7}$ (Krasovskii, 1963). Indeed, $\dot{V}=0$ on $K$ and $\dot{V}>0$ outside the manifold, because the dissipation is complete, i.e., $\tilde{Q}_{i} \dot{q}^{i}<0$ for $\dot{\mathbf{q}} \neq \mathbf{0}$. Since in the vicinity of the origin, $\Pi<0, V$ is positive when $\dot{\mathbf{q}}=\mathbf{0}$. The manifold $K$ does not contain whole trajectories, since for $\dot{\mathbf{q}}=\mathbf{0}$ and $\mathbf{q} \neq \mathbf{0}$ the Lagrange equations (2.3) reduce to $\partial \Pi /\left.\partial q^{k}\right|_{\mathbf{q} \neq \mathbf{0}}=0$, which is impossible for an isolated equilibrium. Therefore, the application of Krasovsky's theorem leads to instability.

This theorem implies that if a nonzero degree of instability equilibrium is stabilized with gyroscopic forces as in Fig. 5(a), then the stability is destroyed by an introduction of arbitrarily small dissipative forces. This result is illustrated in Fig. 5(c) for the following system:

$$
\begin{aligned}
& \ddot{q}_{1}+g \dot{q}_{2}+d \dot{q}_{1}+c_{1} q_{1}=0, \\
& \ddot{q}_{2}-g \dot{q}_{1}+d \dot{q}_{2}+c_{2} q_{2}=0,
\end{aligned}
$$

which has the equilibrium $(\mathbf{q}, \dot{\mathbf{q}})=(\mathbf{0}, \mathbf{0})$. If $c_{i}<0, i=1,2$, it has an even degree of instability equal to 2 . This equi-

\footnotetext{
${ }^{7}$ Krasovsky's theorem states the following: "If for a system $\mathrm{d} \mathbf{x} / \mathrm{d} t=X(\mathbf{x})$ one can find a function $V$ such that its derivative $\dot{V}$ satisfies two conditions, (1) $\dot{V}>0$ outside $K$, and (2) $\dot{V}=0$ on $K$, where $K$ is a manifold of points not containing whole trajectories for $0 \leqslant t<\infty$, and also if in any vicinity of the origin one can find points at which $V>0$, then the origin is unstable." This theorem is a generalization of Lyapunov's theorem on instability. Indeed, Lyapunov's version requires the existence of a function $V: D \rightarrow \mathbb{R}$, defined on the domain $D$ containing the equilibrium point $x=0$, such that both $V$ and $\dot{V}$ assume the same sign in the vicinity of $x=0$. That is, one can regard $V$ as a counterpart of the standard Lyapunov function used in stability theorems (Khalil, 2001). Krasovsky's theorem relaxes Lyapunov's conditions on $V$, as is seen from the formulation of the theorem. A similar relaxation of the conditions on the Lyapunov function in the stability theorems is given in LaSalle's invariance principle (Khalil, 2001).
}

librium point can be spectrally stabilized in the absence of dissipation, $d=0$, by adding gyroscopic forces provided that $|g|>\sqrt{-c_{1}}+\sqrt{-c_{2}}$. The addition of a dissipative force, $d>0$, destabilizes the system regardless of its stability under the action of gyroscopic forces.

The dynamics of this example can be interpreted, for instance, using the basic theory of the gyroscope, as that of the linearized equations of a Lagrange top, which is familiar to those who have spun a toy top or a ball on their fingertip. Even when the top is deflected from the unstable (vertical) equilibrium position and is thus under the action of destabilizing forces, a fast enough rotation makes it move in a direction perpendicular to the destabilizing force and to precess.

However, if the degree of instability is odd, as in Fig. 5(b), then the mechanism described above for gyroscopic stabilization does not work-gyroscopic stabilization is prohibited by another theorem:

Theorem 4 (Thomson-Tait-Chetayev). If the system $\ddot{\mathbf{q}}$ $+C_{0} \mathbf{q}=0$ has an odd degree of instability, then gyroscopic stabilization of the equilibrium is impossible.

Proof. Here we consider system (2.4), when only potential and gyroscopic forces are present,

$$
\ddot{\mathbf{q}}+S \dot{\mathbf{q}}+C_{0} \mathbf{q}=0,
$$

so that the spectral stability analysis leads to the characteristic equation

$$
\left|\begin{array}{cccc}
\lambda^{2}+c_{1} & g_{12} \lambda & \ldots & g_{1 n} \lambda \\
g_{21} \lambda & \lambda^{2}+c_{2} & \ldots & g_{2 n} \lambda \\
\vdots & \vdots & & \vdots \\
g_{n 1} \lambda & g_{n 2} \lambda & \ldots & \lambda^{2}+c_{n}
\end{array}\right|=0,
$$

where $g_{i j}$ are the entries of the gyroscopic matrix $S, c_{i}$ are the stability coefficients, and $\lambda$ 's are the eigenvalues. Obviously, the determinant is a polynomial of the form

$$
\lambda^{2 n}+\cdots+a_{2 n}=0,
$$

where the last term is constant, i.e., independent of $\lambda$, and equals the product of diagonal elements of the potential matrix $C_{0}, a_{2 n}=c_{1} \times \cdots \times c_{n}$, that is independent of gyroscopic forces. Note that this product $a_{2 n}$ is nonzero since the equilibrium is isolated and negative since the degree of instability is odd by assumption of the theorem. Therefore, the function $\operatorname{Det}(\lambda)=\lambda^{2 n}+\cdots+a_{2 n}$ has two limiting values: $\operatorname{Det}(0)=a_{2 n}<0$ and $\operatorname{Det}(\lambda)$ $\rightarrow+\infty$. As a result, $\operatorname{Det}(\lambda)$ crosses the $\lambda$ axis at the point where $\lambda$ has a positive real part, and thus the characteristic equation has at least one eigenvalue with a positive real part. This yields instability according to Lyapunov's theorem on stability in the first approximation regardless of the presence and amplitude of gyroscopic forces.

Theorem 4 provides a necessary condition for stability and a sufficient condition for instability in the framework of the spectral approach; sharper conditions for gyroscopic stabilization have been discussed, for example, by Merkin (1974) and Hryniv et al. (2000). A geometric interpretation of Theorem 4 is suggested by the 
Lagrange-Dirichlet criterion (Theorem 2), namely, by looking at the second variation of the Hamiltonian, which in this case is simply Eq. (2.5) and results in

$$
\delta^{2} H=\left(\begin{array}{cc}
C_{0} & 0 \\
0 & \text { id }
\end{array}\right) .
$$

Apparently this second variation is indefinite and thus does not guarantee the stability of this system, but does not disprove it either since the Lagrange-Dirichlet criterion is not necessary. However, it shows that the energy surface has a saddle point. Now, the advantage of separability of the Hamiltonian in this case (i.e., it is simply kinetic plus potential energy) allows one to use the converse to the Lagrange criterion, which provides a sharp result on the instability: if the second variation is nondegenerate but indefinite, then one has spectral and hence Lyapunov instability. Last, we note that the determinant of the second variation $\delta^{2} H$ is simply the term $a_{2 n}$ in the characteristic polynomial, thus establishing a natural link to the spectral proof of Theorem 4.

It is also interesting to understand the effect of gyroscopic stabilization from an energetic and thus geometric point of view. The discussion below refers to a wide class of systems, but for illustrative purposes and in order to establish a connection to the subsequent sections, we treat again one of the key examples, namely, the Lagrange top problem (1.1), but with a different emphasis,

$$
\begin{aligned}
& \ddot{x}+2 g \dot{y}+c_{1} x=0, \\
& \ddot{y}-2 g \dot{x}+c_{2} y=0 .
\end{aligned}
$$

While the stability of this system can be studied by the spectral method as was done above, here we introduce the notion of the amended potential to account for the effect of gyroscopic forces. The energy of this system is

$$
E=\frac{1}{2}\left[\dot{x}^{2}+\dot{y}^{2}\right]+\frac{1}{2}\left[c_{1} x^{2}+c_{2} y^{2}\right],
$$

since the gyroscopic forces do not work, and the Lagrangian is given by

$$
L=\frac{1}{2}\left[\dot{x}^{2}+\dot{y}^{2}\right]-\frac{1}{2}\left[c_{1} x^{2}+c_{2} y^{2}\right]+g[\dot{x} y-\dot{y} x] .
$$

Effectively, the Lagrangian has the structure of a rotating system with angular velocity $\boldsymbol{\omega}=g \mathbf{k}$ since $(\dot{\mathbf{x}}+\boldsymbol{\omega}$ $\times \mathbf{x})^{2} / 2$ naturally leads to the kinetic energy and gyroscopic terms in Eq. (3.8). Also, to understand the origin of the term $\dot{\mathbf{x}}+\boldsymbol{\omega} \times \mathbf{x}$, consider the transformation from the inertial frame $\mathbf{x}^{\prime}$ to a rotating frame $\mathbf{x}$, and this will recover $\dot{\mathbf{x}}^{\prime}=\dot{\mathbf{x}}+\boldsymbol{\omega} \times \mathbf{x}$. We will observe this behavior in the restricted three-body problem as well as in the Kepler problem later. Two different physical systems-a planar oscillator on a rotating plate and a charged spherical pendulum in a magnetic field-have been discussed by Bloch et al. (2004). With this Lagrangian, momenta are $p_{x}=\dot{x}+g y, p_{y}=\dot{y}-g x$, and the corresponding Hamiltonian $H=E \circ(\mathbb{F} L)^{-1}$ is given by

$$
\begin{aligned}
H= & \frac{1}{2}\left[p_{x}^{2}+p_{y}^{2}\right]+g\left[p_{y} x-p_{x} y\right] \\
& +\frac{g^{2}}{2}\left[x^{2}+y^{2}\right]+\frac{1}{2}\left[c_{1} x^{2}+c_{2} y^{2}\right],
\end{aligned}
$$

where $\mathbb{F} L: T \mathrm{Q} \rightarrow T^{*} \mathrm{Q}$ is a Legendre transform from $q^{i}, \dot{q}^{j}$ to $q^{i}, p_{j}$. First consider the symmetric case, $c_{1}=c_{2}=c$, and we are interested in the unstable potential energy, $c<0$. Let $\mathbf{q}=(x, y), \mathbf{p}=\left(p_{x}, p_{y}\right)$. It is clear that the second variation $\delta^{2} H=H_{\mathbf{z z}}$, where $\mathbf{z}=(\mathbf{q}, \mathbf{p}) \in T^{*} \mathrm{Q}$, is indefinite,

$$
H_{\mathbf{z z}}=\left(\begin{array}{cccc}
c+g^{2} & 0 & 0 & g \\
0 & c+g^{2} & -g & 0 \\
0 & -g & 1 & 0 \\
g & 0 & 0 & 1
\end{array}\right) .
$$

This can also be seen more easily from the simple formula (3.7) for $E$ in terms of $(\mathbf{q}, \dot{\mathbf{q}}) \in T \mathrm{Q}$ (the definiteness of $\delta^{2} H$ does not depend on the choice of variables). Therefore, should one use the Lagrange-Dirichlet criterion, which is valid for the general nonseparable Hamiltonian, one cannot draw a conclusion concerning the stability of this system (the criterion guarantees stability only if the second variation is definite, but if it is indefinite the system could be stable or unstable). However, from a spectral analysis we know that the system is stable, provided that $|g|>\sqrt{-c}$. This interesting behavior follows from the existence of a conserved quantity, which can easily be found from the Noether theorem as a consequence of $S^{1}$ symmetry,

$$
J=p_{x} y-p_{y} x=\dot{x} y-\dot{y} x+g\left(x^{2}+y^{2}\right)=\text { const },
$$

which has the meaning of conservation of angular momentum. In this symmetric case, the natural treatment can be done in the polar coordinates, $x=r \cos \phi$ and $y$ $=r \sin \phi$, so that the conserved quantities are

$$
E=\frac{1}{2}\left(\dot{r}^{2}+r^{2} \dot{\phi}^{2}\right)+\frac{c}{2} r^{2}, \quad J=(g-\dot{\phi}) r^{2}=\mu .
$$

Obviously, in these coordinates $\phi$ is a cyclic variable, i.e., the natural mechanical system reduces to the simple mechanical system, and eliminating $\dot{\phi}$ yields

$$
E=\frac{1}{2}\left\{\dot{r}^{2}+r^{2}\left(g-\frac{\mu}{r^{2}}\right)^{2}\right\}+\frac{c}{2} r^{2},
$$

and the part dependent on coordinates only, $V_{\mu}=c r^{2} / 2$ $+r^{2}\left(g-\mu / r^{2}\right)^{2} / 2$, can be regarded as an effective (amended) potential. In particular, if $\mu=0$, then $V_{\mu}=(c$ $\left.+g^{2}\right) r^{2} / 2$, which coincides with the potential part of the Hamiltonian (3.9) for $c_{1}=c_{2}=c$, and on the basis of the Lagrange-Dirichlet criteria, guarantees stability if $g$ $>\sqrt{-c}$, which is consistent with the results of the spectral analysis. This illustrates the basic idea of exploiting symmetries in analyzing the stability, and a more general theory-the energy-momentum method-will be discussed in Sec. V.C. Note also that the same conclusions can be drawn by reformulating the problem as a con- 
strained one with Lagrange multiplier $H_{\mu}=H+\lambda(J-\mu)$. We refer the reader to the illuminating discussion in the appendix to Wang et al. (1991).

In the nonsymmetric case, i.e., when $c_{1} \neq c_{2}$, there is no continuous symmetry and corresponding conservation law. However, we know that the spectral stability analysis yields the condition $|g|>\left(\sqrt{-c_{1}}+\sqrt{-c_{2}}\right) / 2$, while a naive identification of the effective potential from Eq. (3.9) as $\frac{g^{2}}{2}\left[x^{2}+y^{2}\right]+\frac{1}{2}\left[c_{1} x^{2}+c_{2} y^{2}\right]$ suggests the condition $|g|>\max _{i} \sqrt{-c_{i}}$, which is not as sharp as the spectral one. Therefore, the problem of defining the effective potential for nonsymmetric systems is not resolved yet, but its use is quite apparent.

With the above understanding, the physical interpretation of Theorem 4 becomes simple. Having again the Lagrange top in mind, but when the potential function surface is as in Fig. 5(b), then the gyroscopic force changes its direction passing from the concave to the convex part of the potential function, which leads to a change of the angular momentum, namely, its component about the vertical axis. Finally, it is interesting to note that there are "exceptions" to Theorem 4, as illustrated by the following example.

Example (stability of a disk rolling along a straight line). Consider a perfectly circular disk of mass $m$ and radius $a$ rolling along the $O x_{1}$ axis with angular velocity $\dot{\phi}=\Omega$, as in Fig. 6; this motion is a relative equilibrium. Because of the circular symmetry of the disk, the moment of inertia in the rotating Gxyz-coordinate system is given by $\mathbf{I}=\left(I_{x}, I_{y}, I_{z}\right)=(A, B, C)$, where $B=A$. We are interested in the first-order perturbations of the Euler angles $(\theta, \psi, \phi)$,

$$
\theta=\frac{\pi}{2}+\theta^{\prime}, \quad \psi=\psi^{\prime}, \quad \dot{\phi}=\Omega \simeq \text { const }
$$

where $\theta^{\prime}$ is a deflection of the disk from its vertical plane, and $\psi^{\prime}$ is a deflection from its straight trajectory along the $O x_{1}$ axis. This produces a simplified system

$$
\begin{aligned}
& \ddot{\theta}^{\prime}+\frac{C+m a^{2}}{A+m a^{2}} \Omega \dot{\psi}^{\prime}-\frac{m g a}{A+m a^{2}} \theta^{\prime}=0, \\
& \ddot{\psi}^{\prime}-\frac{C}{A} \Omega \dot{\theta}^{\prime}=0,
\end{aligned}
$$

which apparently has an unstable potential energy and one negative stability coefficient (one degree of instability), but the gyroscopic force can stabilize the motion provided

$$
\Omega^{2} \geqslant \frac{A}{C} \frac{m g a}{C+m a^{2}} .
$$

Even though the equilibrium solution-the disk rolling with a constant speed $\Omega a$-is a relative equilibrium, the resulting linearized equations are accounted for by an operator with constant coefficients and thus the classical analysis should be readily applicable. The gyroscopic stabilization of an equilibrium in systems with an odd degree of instability seems to be impossible in view of

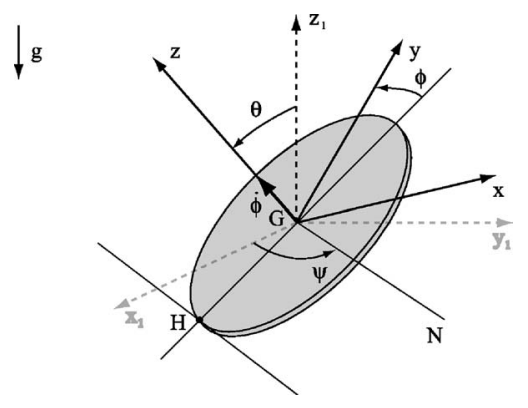

FIG. 6. Geometric setup. Gxyz is "frozen" in the disk. $G z$ is perpendicular to the disk plane. $G N$ is a line of nodes (cross section of planes $x_{1} G y_{1}$ and $x O y$ ), which is parallel to the surface of contact. Line $H N^{\prime}$ is tangent to the disk at point $H$.

the Thomson-Tait-Chetayev Theorem 4 on the necessary condition for gyroscopic stabilization. However, the example of a rolling disk does not contradict this theorem: the gyroscopic stabilization is possible in this case in view of criticality, i.e., when at least one of the stability coefficients is zero, which is not accounted for by the classical theorems.

\section{Application 1: Radiation-induced instability}

As an application of the Thomson-Tait-Chetayev result (Theorem 3), we consider the work of Hagerty et al. $(1999,2003)$, in which radiation-induced instability is discussed. We show here that this type of instability can be accounted for using Theorem 3 .

The physical setup is modeled by a finite-dimensional system-for example, a two-degrees of freedom gyroscopic systems analogous to the Lagrange top without dissipation (1.1) - coupled to an infinite-dimensional one, such as the wave equation $\partial^{2} w / \partial t^{2}-c^{2} \partial^{2} w / \partial \xi^{2}=0$, that is responsible for a process of wave radiation. Coupling of this type is important in various physical systems: for the origin of this model, we refer the reader to the work of Soffer and Weinstein (1999). The resulting governing system has the form

$$
\begin{aligned}
& \ddot{x}+g \dot{y}+\alpha x=\gamma \int_{0}^{t}[x(s)+y(s)] \mathrm{d} s, \\
& \ddot{y}-g \dot{x}+\beta y=\gamma \int_{0}^{t}[x(s)+y(s)] \mathrm{d} s,
\end{aligned}
$$

where the definitions on the left-hand side are the same as in Eq. (1.1) and the right-hand side describes the effects of radiation through the wave propagation process, whose form originates from the coupling $\gamma \int_{\mathrm{R}} \chi(\xi) w(\xi, t) \mathrm{d} \xi$ to the finite-dimensional dynamics with the distribution $\chi(\xi)$. The work of Hagerty et al. (1999) establishes the Lyapunov instability of this system, which is caused by the presence of radiation even when the mechanical part (i.e., the left-hand side) of Eq. (3.16) is spectrally stable, say gyroscopically stabilized.

The proof in Hagerty et al. (1999) is based on differentiation of the above system followed by a direct analy- 
sis. On the other hand, in our approach, we introduce another variable, $z=\int_{0}^{t}[x(s)+y(s)] \mathrm{d} s$, so that the preceding system reads

$$
\ddot{\phi}+G \dot{\phi}+C \phi=\mathbf{0}, \quad \phi=[x, y, z]^{T},
$$

where $G$ and $C$ are given by

$$
G=\left[\begin{array}{ccc}
0 & -g & 0 \\
g & 0 & 0 \\
-1 & -1 & 0
\end{array}\right], \quad C=\left[\begin{array}{ccc}
\alpha & 0 & -\gamma \\
0 & \beta & -\gamma \\
0 & 0 & 0
\end{array}\right] .
$$

Introducing the change of variables $\phi^{\prime}=A \phi$, where $A$ is such that $A C A^{-1}$ is diagonal, we arrive at

$$
\ddot{\phi}^{\prime}+\tilde{G} \dot{\phi}^{\prime}+\tilde{C} \phi^{\prime}=\mathbf{0}, \quad \tilde{C}=\left[\begin{array}{ccc}
\alpha & 0 & 0 \\
0 & \beta & 0 \\
0 & 0 & 0
\end{array}\right],
$$

where

$$
\tilde{G}=\left[\begin{array}{ccc}
\frac{\gamma}{\alpha} & \frac{\gamma}{\alpha}-g & \frac{\gamma^{2}}{\alpha^{2}}+\frac{\gamma}{\beta}\left(\frac{\gamma}{\alpha}-g\right) \\
\frac{\gamma}{\beta}+g & \frac{\gamma}{\beta} & \frac{\gamma^{2}}{\beta^{2}}+\frac{\gamma}{\alpha}\left(\frac{\gamma}{\beta}+g\right) \\
-1 & -1 & -\frac{\gamma}{\alpha}-\frac{\gamma}{\beta}
\end{array}\right],
$$

where the new matrix $\tilde{G}$ has nondegenerate symmetric (dissipative) and antisymmetric (gyroscopic) parts. Therefore, if the system with $\tilde{G}=0$ is unstable, then adding arbitrary gyroscopic and dissipative forces leaves it unstable in accordance with Theorem 3. Note that even though the classical Thomson-Tait-Chetayev theory was developed for the noncritical case (i.e., all the stability coefficients are nonzero), its physical implications are wider and in many situations, including this one, an examination of the proofs shows that the theorems are still true and yield correct predictions.

\section{Application 2: The Levitron}

The invention of a levitating magnetic object by Harrigan (1983) overcame the taboo imposed by the Earnshaw theorem ${ }^{8}$ (Earnshaw, 1842) and received some

\footnotetext{
${ }^{8}$ This theorem states that a collection of point charges cannot be maintained in an equilibrium configuration solely by the electrostatic interaction of the charges. In general, this theorem applies to static forces $\mathbf{F}(x)$, which are functions of position-gravitation, electrostatic, and magnetostatic. Note that these fields are always divergenceless, $\operatorname{div} \mathbf{F}=0$. The proof of the theorem is a simple consequence of Gauss's theorem. Indeed, at a point of equilibrium the force is zero, and if the equilibrium is stable the force must point in toward the point of equilibrium on some small sphere around the point. However, by Gauss's theorem, $\int \mathbf{F}(x) \mathrm{d} S=\int \operatorname{div} \mathbf{F} \mathrm{d} V$, the integral of the radial component of the force over the surface must be equal to the integral of the divergence of the force over the volume inside, which is zero.
}

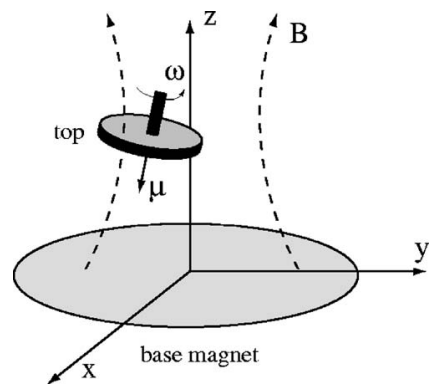

FIG. 7. Schematics of the Levitron.

resonance in the literature (Berry, 1996; Simon et al., 1997). However, a closer look at this problem reveals that Harrigan had very strong intuitive reasons that are, in fact, supported by the Thomson-Tait-Chetayev theory. As discussed in Sec. II, this theory allows the possibility of gyroscopic stabilization of an unstable system (with a nonzero degree of instability), a fact that is used in numerous engineering applications (such as a monorail car, etc.). It is curious that despite the existence of this classical theory, the explanation of stabilization in the literature was based on an approximate adiabatic invariants theory [cf. Berry (1996) and Simon et al. (1997)]. As discussed below, a simpler explanation of the stability of the Levitron can be based on the ThomsonTait-Chetayev theory.

The dynamics of a point magnetic dipole of strength $\mu$ and mass $M$ in an axisymmetric magnetic field $\mathbf{B}$, as shown in Fig. 7, is governed by torque and force balance (angular and linear momenta respectively) as follows:

$$
\begin{aligned}
& \mathrm{d}_{t} \boldsymbol{\mu}=\frac{\mu}{I \omega} \boldsymbol{\mu} \times \mathbf{B}, \\
& M \mathrm{~d}_{t}^{2} \mathbf{r}=\nabla(\boldsymbol{\mu} \cdot \mathbf{B})-M g \hat{z} .
\end{aligned}
$$

The magnetic field can be represented in the neighborhood of its axis of symmetry by means of a Taylor series expansion,

$$
\begin{aligned}
& B_{z}=B_{0}+S z+K z^{2}-\frac{1}{2} K r^{2}+\cdots, \\
& B_{r}=-\frac{1}{2} S r-K r z+\cdots .
\end{aligned}
$$

With the nondimensionalization

$$
\begin{aligned}
& \boldsymbol{\mu} \rightarrow a \boldsymbol{\mu}, \quad \mathbf{r} \rightarrow \alpha \mathbf{r}, \quad t \rightarrow \gamma t ; \\
& \frac{a}{\alpha}=\frac{\omega_{p}}{\omega} \frac{\mu^{2} M}{I}, \quad \gamma=\sqrt{\frac{M}{I \omega}}, \quad \omega_{1}=\gamma \frac{a}{\alpha} \frac{S}{2 M}, \quad \omega_{2}=\gamma \omega_{p},
\end{aligned}
$$

the linearized equations for $\mathbf{z}=\left[x, y, \mu_{x}, \mu_{y}\right]^{T}$ written in component form become 


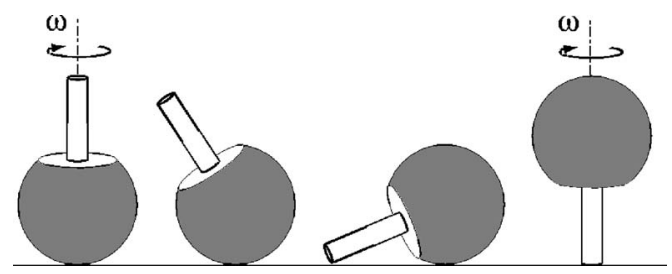

FIG. 8. Inversion of a tippe-top.

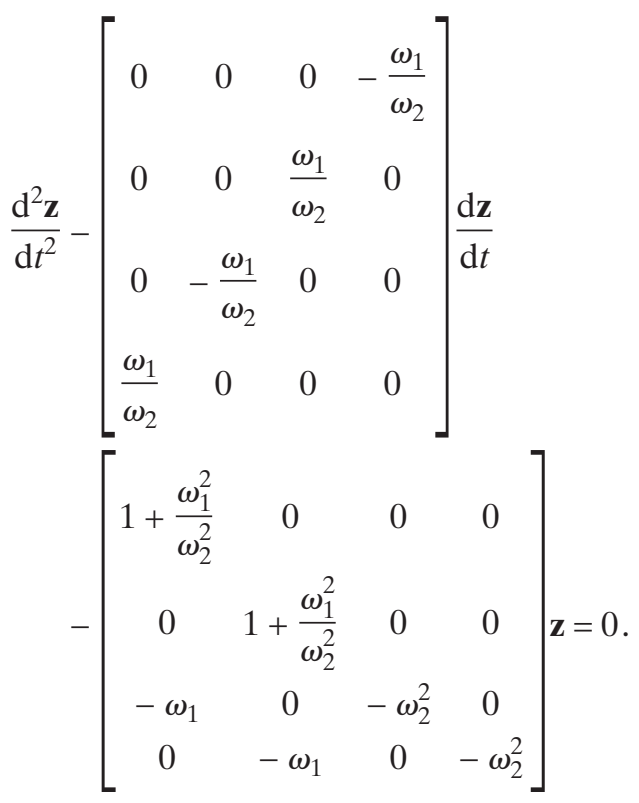

It is obvious that the degree of instability of this system is even, so that one can expect stabilization for a certain ratio of frequencies $\omega_{1}$ and $\omega_{2}$, since the stabilization is achieved only at a certain amplitude of gyroscopic force.

The dissipative effects in a spinning top are known to be crucial since they determine the finite lifetime of a stable levitation (Simon et al., 1997). However, those effects are, in general, very complicated due to the top's finite size, conductivity, magnetization, interaction with air, etc. As shown by Krechetnikov and Marsden (2006), eddy currents introduce both types of nonconservative forces, i.e., dissipative and positional. The presence of both types of nonconservative forces implies that the Levitron will always be unstable (though the characteristic time of instability can be large) unless these dissipative effects are compensated for with external pumping of energy, as is often done in gyroscopic systems (Merkin, 1997).

\section{A few more applications}

Concluding the discussion of the Thomson-TaitChetayev theory, we mention a couple of other interesting physical phenomena where effects of dissipation play the crucial role. In the first one-the inversion of a tippetop shown in Fig. 8-the role of dissipation was the subject of a long debate until Cohen (1977) established the contention of the earlier works in 1950s that it is the sliding frictional forces acting at the point of contact between the top and the plane of support that are responsible for the inversion. The main difficulty of accounting for dissipation in this problem was that the usual Cou- lomb law leads to a nonlinear term, which disappears in the linearized equations used to study the instability, until O'Brien and Synge (1953) suggested using a viscous friction linear in the sliding velocity. The relative complexity of this problem has led to various numerical studies aiming to prove the destabilizing effect of dissipation, such as Cohen (1977), Kane and Levinson (1978), and Or (1994). The history of this problem is well discussed in these references and by Ebenfeld and Scheck (1995).

However, speaking of the most fundamental cause for (linear) instability, one can use the Thomson-TaitChetayev argument and avoid lengthy computations. To achieve this, we will omit bulky equations, but rather provide more insightful analysis applicable in many other situations. In particular, we refer to the linearized equations of motion given by Or (1994) [Eqs. (11) in that reference], which in the absence of friction after some algebra take the form

$$
\ddot{\mathbf{q}}+(S+D) \dot{\mathbf{q}}+(C+P) \mathbf{q}=0 .
$$

Even though the problem is nonholonomic (in the absence of dissipation) and thus non-Hamiltonian, the energy is conserved, and therefore both the nonlinear and linearized equations are conservative. It is notable that it appears that Eq. (3.18) contains dissipative and positional forces. However, because of the conservative nature of these equations, there should exist a linear transformation $\mathbf{q} \rightarrow \mathbf{T} \tilde{\mathbf{q}}$ such that system (3.18) transforms into Eq. (2.4) without nonconservative forces,

$$
\ddot{\tilde{\mathbf{q}}}+\mathbf{T}^{-1}(S+D) \mathbf{T} \dot{\tilde{\mathbf{q}}}+\mathbf{T}^{-1}(C+P) \mathbf{T} \tilde{\mathbf{q}}=0,
$$

where $\mathbf{T}^{-1}(C+P) \mathbf{T}$ is made diagonal. Since the linearized dynamics is conservative, the term $\mathbf{T}^{-1}(S+D) \mathbf{T}$ should be necessarily skew symmetric (gyroscopic). Therefore, the addition of sliding friction should lead to terms symmetric in $\dot{\tilde{\mathbf{q}}}$ and produce instability in accordance with Thomson-Tait-Chetayev Theorem 3, thus explaining the dissipation-induced instability of a tippe-top.

Nowadays, the problem of stability of a tippe-top is well understood globally as well, after the work of Ebenfeld and Scheck (1995). Namely, they demonstrated that the only asymptotic solutions, to which the spinning tippe-top could tend if they are found to be stable, are (i) rotational, when the top rotates about a vertical axis through a fixed point on the plane, (ii) tumbling, when the motion of the spinning top rolls over the plane without sliding, and (iii) spinning with sliding over the plane of support. Those solutions are also proved to be the limit sets of the solution for the general problem and arbitrary initial conditions. Ebenfeld and Scheck also established the conditions for which each of the constant energy solutions in the limit set is stable, so that there exists only one trajectory tending to this solution as $t$ $\rightarrow \infty$ for arbitrary initial conditions. Another problem, which is often related to the tippe-top-namely, the rattleback (Walker, 1979)—does not seem to have the same profound effect of dissipation (Borisov and Mamaev, 2003). 


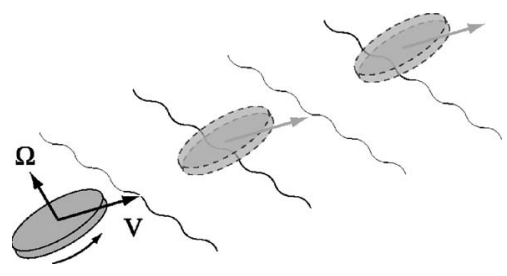

FIG. 9. Skipping stone.

Another fascinating physical system-skipping stones (cf. Fig. 9) -also illustrates Theorem 3. However, besides the oversimplified phenomenological theory (Bocquet, 2003), there is no adequate (even approximate finite-dimensional) description of this problem, which would allow one to understand its physics better. It is notable that skipping stones (cf. Fig. 9) require an initial spin $\Omega$ for gyroscopic stabilization (Bocquet, 2003), and their interaction with the underlying fluid leads to dissipation. Therefore an approximate, finite-dimensional, description should fit the universal picture introduced in this work. In particular, the known fact of gyroscopic stabilization and the presence of dissipation in this problem allows one to conclude that the skipping stone will always be unstable in accordance with experience and Theorem 3; as in the Levitron, depending on the details of the particular situation, the characteristic time of instability can be large.

To illustrate the fact that dissipation-induced instabilities are encountered from microscopic to astronomical scales, we appeal to the classical (planar) circular restricted three-body problem following the work of Murray (1994). This problem concerns the motion of a test particle moving under the gravitational effect of two masses $m_{1}$ and $m_{2}$, which in turn move in circular orbits about their common center of mass and are not influenced by the motion of the particle. The motion is considered in a coordinate system rotating about the common center of mass with the same frequency as the two masses so that both of them lie on the $x$ axis with coordinates $\left(-\mu_{2}, 0\right)$ and $\left(\mu_{1}, 0\right)$, where $\mu_{i}=m_{i} /\left(m_{1}+m_{2}\right)$. The resulting equations of motion (Murray, 1994) are

$$
\begin{aligned}
& \ddot{x}-2 \dot{y}=\frac{\partial U}{\partial x}+F_{x}, \\
& \ddot{y}+2 \dot{x}=\frac{\partial U}{\partial y}+F_{y},
\end{aligned}
$$

where

$$
U=\frac{\mu_{1}}{r_{1}}+\frac{\mu_{2}}{r_{2}}+\frac{1}{2}\left[x^{2}+y^{2}\right],
$$

with $r_{1}^{2}=\left(x+\mu_{2}\right)^{2}+y^{2}, r_{2}^{2}=\left(x-\dot{\mu}_{1}\right)^{2}+y^{2}$. In the absence of drag, $\mathbf{F}=\mathbf{0}$, system (3.20) possesses one integral of motion, namely, the Jacobi integral $C=2 U-\dot{x}^{2}-\dot{y}^{2}$, which naturally defines the zero velocity curves, some of which are shown in Fig. 10.

Figure 10 also indicates the location of the five Lagrangian equilibrium points, three collinear ones $L_{1-3}$

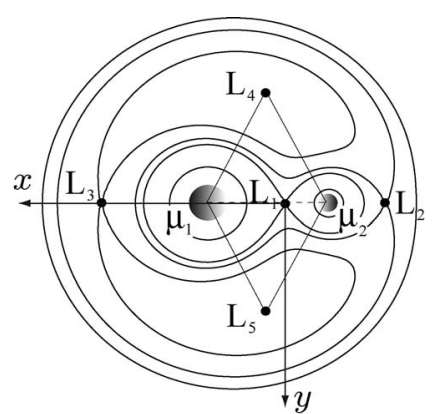

FIG. 10. Restricted three-body problem: critical zero velocity curves and Lagrangian equilibrium points.

and two triangular $L_{4-5}$. From the classical stability analysis, it is known that the $L_{4}$ and $L_{5}$ points are spectrally stable provided $\mu_{1} \mu_{2}<1 / 27$, while the remaining points are unstable. The presence of drag in general changes the location of the equilibrium points, but of course does not make the stability analysis meaningless. In the case of simple nebular drag when the force is proportional to the velocity of the particle in the rotating frame, $\mathbf{F}=k(\dot{x}, \dot{y})$, the locations of the equilibrium points are not affected and, as follows from the Thomson-Tait-Chetayev Theorem 3, the stability of triangular equilibrium points $L_{4}$ and $L_{5}$ is destroyed. The same conclusion applies to more realistic drag forces, including radially dependent inertial drag forces, $\mathbf{F}$ $=k(\dot{x}, \dot{y}) r^{n}$, and Poynting-Robertson light drag, which is caused by the nonisotropic reemission of radiation absorbed by the test particle.

\section{B. Merkin theory}

The counterpart of the Thomson-Tait-Chetayev Theorem 3 for nonconservative positional forces is the following.

Theorem 5 (Merkin). The introduction of nonconservative positional forces [that is, the skew-symmetric matrix $P$ in Eq. (2.4) is nonzero] into a stable purely potential system, $\ddot{\mathbf{q}}+C_{0} \mathbf{q}=0$, with equal frequencies destroys the stability of the equilibrium regardless of the form of the nonlinear terms.

Proof. Consider the following system:

$$
\ddot{\mathbf{q}}+C \mathbf{q}+P \mathbf{q}=F(\mathbf{q}), \quad C=c E, \quad P^{T}=-P .
$$

The potential part $C$ is a diagonal matrix with equal eigenvalues $c$ and the nonconservative part $P$ is a skewsymmetric matrix. From the corresponding characteristic equation $\operatorname{det}\left[E\left(\lambda^{2}+c\right)+P\right]=0$ we see that $\lambda^{2}+c$ is imaginary, so that $\lambda$ is unstable. It is notable that the second variation of the Hamiltonian (when $P \equiv 0$ ) is positive definite at the origin $\delta^{2} H=c^{n}$, where $n$ is the system dimension.

For the history and other important results, we refer the reader to Zajac (1964), Merkin (1974, 1997), Agafonov (2002), and Seyranian and Mailybaev (2003). To illustrate Theorem 5, consider system (1.2). A study of the corresponding characteristic equation shows that 


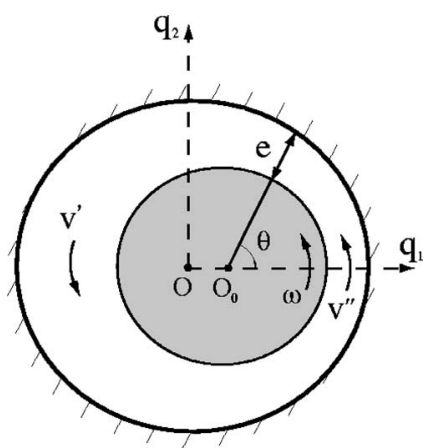

FIG. 11. Rotating shaft geometry (Kapitsa, 1939).

the addition of nonzero, nonconservative, positional forces (that is, $p \neq 0$ ) to a stable system (that is, with zero degree of instability) with equal frequencies makes it unstable, as shown in Fig. 5(d). Note that the second variation of the Hamiltonian of the original system is positive definite at the origin.

\section{Application 1: Rotating shafts}

As a classical illustration of dissipation-induced instability due to positional forces, we consider the problem of rotating shaft, discussed in the Introduction and originally treated by Kapitsa (1939). Since the dissipative nature of these forces should be clear from the physical setup, we consider the dynamics of the perturbation only. Let the rotor rotate with an angular velocity $\omega$ in the ring housing with the space between them filled with a hydrodynamic medium, as depicted in Fig. 11. If the rotor center $O_{0}$ coincides with the housing center $O$, then the friction induces a breaking moment only since the gas velocity has the same profile along the uniform gap. Now, consider the case in which the center is displaced by a small amount $O O_{0}=q_{1}$ to the right along the $q_{1}$ axis. Since the clearance becomes narrower in the direction of displacement, we have $v^{\prime \prime}>v^{\prime}$ as shown in Fig. 11, which leads to different frictional forces on the right and left sides of the rotor surface. Indeed, since the difference between the peripheral velocity of the rotor and the medium is larger on the right side, then the friction on that side is larger than on the left side, thus inducing a resultant force in the $q_{2}$ direction. Quantitatively, this can be explained as follows. If the clearance between the rotor and the ring is $e_{0}$ when their centers coincide, then assuming that the clearance is much smaller than the rotor radius $R$ in the first approximation, we get $e=e_{0}-q_{1} \cos \theta$. Next, if the average velocity of the medium is $R \omega / 2$ and taking into account that the volume of the medium moving through any cross section remains constant, we arrive at the simple relation ve $=R \omega e_{0} / 2$.

Using these facts, we compute the frictional force $d S$ (per unit length in the third dimension) acting as a peripheral surface element $R d \theta$. Assuming that it is

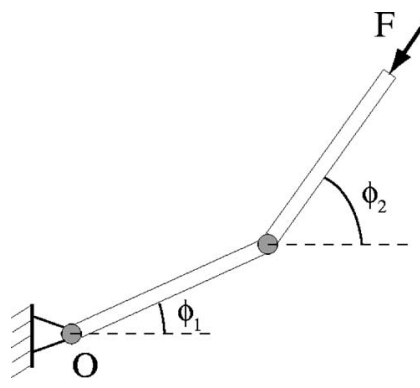

FIG. 12. Schematics of the cantilever-elastic bar.

proportional to the square of the relative velocity ${ }^{9}$ $(R \omega-v)^{2}$ and projecting the force onto the $q_{2}$ axis and integrating over $\theta$, we get

$$
S_{q_{2}}=-\kappa \rho \int_{0}^{2 \pi}(R \omega-v)^{2} \cos \theta \mathrm{d} \theta \sim q_{1} .
$$

Similarly, we can deduce $S_{q_{1}} \sim-q_{2}$, and writing down Newton's second law with the appropriate nondimensionalization we recover system (1.2). It is notable that because of breaking of symmetry of the problem (by displacing the rotor from its center position), skewsymmetric positional forces appear. In this case it is obvious that, should one treat the rotating shaft as a closed system (not only the dynamics of perturbation, but also the dynamics of the basic state-relative equilibrium), these forces would lead to energy dissipation. This is different from the example considered next, when the same linear system (1.2) accounts for the influence of the follower force, which is the force from the external and thus pumps the energy into a system.

\section{Application 2: Secondary instability}

In this section, we continue the discussion of the simultaneous appearance of both types of nonconservative effects and demonstrate their combined effect, which leads to a secondary dissipation-induced instability phenomenon, the appearance of which was discovered by Ziegler (1952) in the context of elastic systems. Here we consider a system of this type, namely, two identical bars of length $l$ and mass $m$, and torsional springs of stiffness $c_{0}$, as shown in Fig. 12. For simplicity, the twobar system is restricted to a plane and not subjected to a gravity field. The moment of inertia of the first bar with respect to the point of attachment $O$ is $J_{1}$, and of the second bar with respect to its center of mass it is $J_{2}$. With these definitions, the kinetic and potential energies of the system for small deflections $\phi_{1,2}$ (linearization) are

$$
T=\frac{1}{2}\left(a_{11} \dot{\phi}_{1}^{2}+2 a_{12} \dot{\phi}_{1} \dot{\phi}_{2}+a_{22} \dot{\phi}_{2}^{2}\right),
$$

\footnotetext{
${ }^{9}$ However, in reality the friction law is a general function of velocity and other variables, so that one can expect the presence of the usual velocity-dependent dissipative forces as well.
} 


$$
\Pi=\frac{1}{2} c_{0} \phi_{1}^{2}+\frac{1}{2} c_{0}\left(\phi_{2}-\phi_{1}\right)^{2}
$$

where $a_{11}=J_{1}+m l^{2}, a_{12}=\frac{1}{2} m l^{2}$, and $a_{22}=J_{2}+\frac{1}{4} m l^{2}$. The resulting Euler-Lagrange equations for angles $\phi_{1}$ and $\phi_{2}$ are

$$
\begin{aligned}
{\left[\begin{array}{ll}
a_{11} & a_{12} \\
a_{12} & a_{22}
\end{array}\right]\left[\begin{array}{l}
\ddot{\phi}_{1} \\
\ddot{\phi}_{2}
\end{array}\right] } & +\left[\begin{array}{ll}
c_{11} & c_{12} \\
c_{12} & c_{22}
\end{array}\right]\left[\begin{array}{l}
\phi_{1} \\
\phi_{2}
\end{array}\right] \\
& +\left[\begin{array}{cc}
0 & p \\
-p & 0
\end{array}\right]\left[\begin{array}{l}
\phi_{1} \\
\phi_{2}
\end{array}\right]=0,
\end{aligned}
$$

where $a_{11}=\frac{4}{3} m l^{2}, a_{12}=\frac{1}{2} m l^{2}$, and $a_{22}=\frac{1}{3} m l^{2} ; c_{11}=2 c_{0}-F l$, $c_{12}=\frac{1}{2} F l-c_{0}, c_{22}=c_{0}$, and $p=\frac{1}{2} F l$. System (3.24) can be reduced to form (2.4) as discussed in Sec. II.B. Note that the follower force contributes to both the positional $P$ and potential $C$ matrices, and thus the situation is slightly more general than the one accounted by Merkin's theorem. The eigenvalue analysis of Eq. (3.24) leads to a quartic equation for $\lambda$, of the form $a \lambda^{4}+b \lambda^{2}$ $+c=0$; this shows that the solution is stable if $\lambda^{2}<0$, that is,

$$
b>0, \quad b^{2}-4 a c>0,
$$

which yields $F<\frac{6}{5}\left(3-\sqrt{\frac{20}{3}}\right) c_{0} / l$. The stability breaks when the magnitude of the follower force exceeds this value and thus the second inequality in Eq. (3.25) changes its sign. This model was studied by Nikolai (1939) as an approximation for the effects occurring due to the outflow of combustion gases in jet engines.

Apparently, in addition to the positional (follower) force $F$, there are regular dissipative forces, so that one can introduce a regular dissipation into the previous example (3.24) and study the effect of two nonconservative forces as in Eq. (2.4), which appear in Eq. (2.4) through matrices $P$ (positional forces) and $D$ (regular dissipation). The origin of $D$ can be due to hydrodynamic friction inside the bar tubes through which there is a flow of liquid and the ejection of which creates a follower force similar to that in a jet engine. Suppose for simplicity that the dissipation matrix $D$ is diagonal with equal diagonal entries of magnitude $\epsilon$, and the magnitude of the follower force is slightly below its critical value; that is, the system is close to buckling.

Performing an asymptotic study of the eigenvalue problem $\boldsymbol{\phi}=\hat{\boldsymbol{\phi}} e^{\lambda t}$ for Eq. (3.24), and writing $\lambda \simeq \lambda_{0}+\epsilon \lambda_{1}$ for the eigenvalues, where $\lambda_{0}$ is the eigenvalue of problem (3.24) without regular dissipation (the stable configuration), it is straightforward to show that

$$
\lambda_{1}=-\frac{c_{0}+e_{1}+\left(a_{11}+a_{22}\right) \lambda_{0}^{2}}{2\left(b+2 a \lambda_{0}^{2}\right)}, \quad e_{1}=2 c_{0}-F l
$$

that is, under assumptions of stability of the nondissipative system (3.25) and in the case when both $a>0$ and $c>0$ (one can show that this is physically realizable), the system experiences an instability, since $\lambda_{1}>0$, for an arbitrary small dissipation $\epsilon$.

\section{On phase-space behavior}

Having discussed the geometric picture of dissipationinduced instabilities in terms of the second variation $\delta^{2} H$ and trajectories in the phase space, we now address another important geometric implication: how does a volume $V(t)$ of some region $D(t)$ in the phase-space change with time under the phase flow

$$
g^{t}:(\mathbf{p}(0), \mathbf{q}(0)) \mapsto(\mathbf{p}(t), \mathbf{q}(t)) .
$$

In the Hamiltonian case, it is known that the phasespace volume is conserved, $D(t)=g^{t} D(t)=$ const, according to Liouville's theorem (Arnold, 1978). More generally, if we have a system of ODEs $\dot{\mathbf{x}}=\mathbf{f}(\mathbf{x})$, then the volume in $\mathbf{x}$ space is conserved if $\operatorname{div} \mathbf{f}=\mathbf{0}$, where $\operatorname{div} \mathbf{f}$ is the time rate of change of the phase-space volume. If $\mathbf{x}_{0}$ is a nonsingular point, then by the local normal form theorem (Arnold, 1973), there exists an orthogonal volume-preserving transformation $\mathbf{y}=\mathbf{y}(\mathbf{x})$, such that this system in the neighborhood of $\mathbf{x}_{0}$ takes the form

$$
\begin{aligned}
& \dot{y}_{1}=\left|\mathbf{f}\left(\mathbf{x}_{0}\right)\right|, \\
& \dot{y}_{j}=\lambda_{j} y_{j}, \quad j=2, \ldots, n,
\end{aligned}
$$

where $\lambda_{j}$ are local Lyapunov exponents. This indicates that one eigenvalue of a flow at a nonsingular point $\mathbf{x}_{0}$ always vanishes and the associated eigenvector points in the direction of the flow. All other Lyapunov exponents are responsible for phase-space volume deformations. Therefore, the divergence can be expressed in terms of the local Lyapunov exponents, $\operatorname{div} \mathbf{f}=\Sigma_{j} \lambda_{j}$.

Next, for simplicity, consider the case of a velocity phase space; then our two key examples from previous sections-the Lagrange top and the rotating shaft—can be treated in a straightforward manner. In the case of an instability induced by regular dissipation, the system for $\mathbf{z}=\left(q_{1}, v_{1}, q_{2}, v_{2}\right)^{T}$ is of the form

$$
\frac{\mathrm{d} \mathbf{z}}{\mathrm{d} t}=\left(\begin{array}{cccc}
0 & 1 & 0 & 0 \\
-c_{1} & -d & 0 & -g \\
0 & 0 & 0 & 1 \\
0 & g & -c_{2} & -d
\end{array}\right) \mathbf{z} .
$$

Therefore, $\operatorname{div} \mathbf{f}=-2 d$, i.e., the volumes shrink with time at the same rate everywhere in the available regions of phase space. This may appear to contradict the fact that the equilibrium of the above system is unstable with $\mathbf{z}(t)$ growing exponentially in time. These two facts can be reconciled by noting that even though the volume $D(t)$ in $\mathbf{z}$ space is decreasing, some of its dimensions are growing in the unstable eigendirections of the operator on the right-hand side of $\dot{\mathbf{z}}=(\mathrm{J}+\mathrm{G}) \nabla_{\mathbf{z}} H$, as depicted in Fig. 13. Here $J$ is a symplectic skew-symmetric operator, and $\mathrm{G}$ is the dissipative part of the operator (metriplectic part). It is worth noting that those unstable eigendirections need not coincide with unstable eigendirections of the second variation $\left.\nabla_{\mathbf{z}}^{2} H\right|_{\mathbf{z}=\mathbf{0}}$.

Next, in the case of an instability induced by positional forces, the system is 
$|q|$

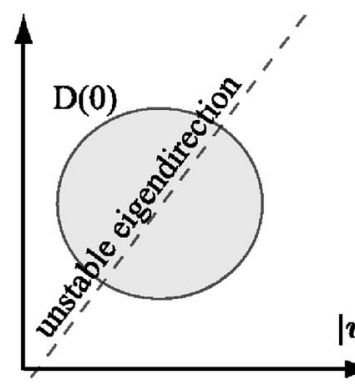

$|q|$

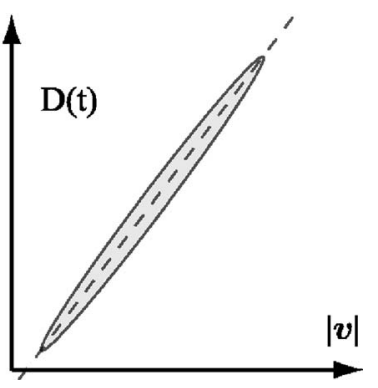

FIG. 13. A cartoon showing the velocity phase-space shrinkage in the case of dissipation-induced instability.

$$
\frac{\mathrm{d} \mathbf{z}}{\mathrm{d} t}=\left(\begin{array}{cccc}
0 & 1 & 0 & 0 \\
-c & 0 & -p & 0 \\
0 & 0 & 0 & 1 \\
p & 0 & -c & 0
\end{array}\right) \mathbf{z} .
$$

From here we immediately see that $\operatorname{div} \mathbf{f}=0$, that is, the volume is conserved even though the system is nonconservative, which illustrates that Liouville's theorem does not have a converse. In terms of the behavior of the phase space, this case is analogous to the Hamiltonian instabilities: the phase-space volume shape is deformed similar to Fig. 13, but its volume is conserved.

\section{Summary and discussion}

In this section, we illustrate the power of the relevant classical theorems in explaining several phenomena currently under discussion in the literature (such as radiation-induced instability, the Levitron, etc.). We also introduce the basic geometric interpretation of the two fundamental destabilization mechanisms, summarized below, and demonstrate their generic simultaneous appearance in physical systems. The latter naturally leads to the notion of secondary dissipation-induced instabilities.

As the structure of the complete fundamental system (2.4) suggests, the two nonconservative destabilizing effects discussed and illustrated in Figs. 5(c) and 5(d) exhaust the most fundamental possibilities for finitedimensional mechanical systems.

The two destabilization mechanisms can be summarized as follows. If a stable equilibrium is formed from an unstable potential energy together with stabilizing gyroscopic forces, then this stability is destroyed by arbitrary dissipative forces. On the contrary, if the stable equilibrium is formed from stable potential forces with equal frequencies alone, then the stability is destroyed by arbitrary nonconservative positional forces. It is notable that these two cases both have antisymmetric coupling in system (2.4), which basically prohibits the construction of a Lyapunov function to prove stability. The instabilities occur in both cases due to breaking of symmetry in the original conservative system (and its phase space) so that the eigenvalues move away from the imaginary axis.
To summarize the geometric observations, we again refer to the two key examples-the Lagrange top and the rotating shaft-discussed in the Introduction. As pointed out by Bloch et al. (1994), the fact that the second variation of the Hamiltonian $H=\frac{1}{2} \dot{\mathbf{z}}^{T} \dot{\mathbf{z}}+\frac{1}{2} \mathbf{z} C \mathbf{z}$ for Eq. (1.1) is indefinite is crucial for the destabilizing effect of dissipation, since the condition necessary for stability of a Hamiltonian system, namely, definite second variation $\delta^{2} H$ in the Lagrange-Dirichlet theorem, is not satisfied. At the same time, it is notable that, in contrast to the Lagrange top example (1.1), the second variation $\delta^{2} H$ for the rotating shaft problem (1.2) is positive definite at the origin and thus the energy of the disturbance eventually grows. ${ }^{10}$ Thus, in the finite-dimensional case, the fundamental difference in the type of nonconservative forces, i.e., regular dissipative versus positional, has a direct impact and goes in parallel with a drastic change in the geometrical picture, i.e., indefinite versus definite second variation,

indefinite $\delta^{2} H \Rightarrow$ dissipative forces destabilize,

definite $\delta^{2} H \Rightarrow$ positional forces destabilize,

where we have summarized the geometric precursor which predetermines the type of nonconservative destabilizing forces. ${ }^{11}$

\section{MOVEMENTS OF EIGENVALUES: GEOMETRY IN SPECTRAL SPACE}

Here we take an alternative look at the dissipationinduced instabilities-instead of the configuration or phase space, we consider the picture in spectral space.

\section{A. Hamiltonian bifurcations}

The first task is to establish a link between the effect of dissipation and bifurcations in the corresponding Hamiltonian systems, which may appear to be a stretch since the concepts of stability in dissipative and conservative systems are quite different. Namely, the equilibrium in a dissipative system is stable if all eigenvalues of the linear operator have negative real parts, as follows from Lyapunov's theorem. On the contrary, in a Hamiltonian system a necessary condition for stability is that the entire spectrum lies on the imaginary axis, since the spectrum of conservative systems is always symmetric with respect to both real and imaginary axes, and therefore is present in either doublets or quartets. Since the

\footnotetext{
${ }^{10}$ This, however, does not prohibit having $\dot{H}<0$ on certain portions of the trajectory, as can be readily seen from the energy production rate equation: $\dot{H}=p\left(z_{1} \dot{z}_{2}-z_{2} \dot{z}_{1}\right)$. These portions of a motion correspond to the trajectories that are temporarily heading toward equilibrium.

${ }^{11}$ Some other possibilities might be observed in, for instance, the degenerate case when the second variation vanishes or in the case in which a finite-amplitude instability takes place, which we do not discuss here.
} 


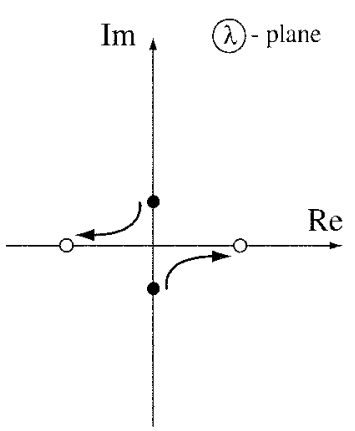

(a) Steady-state bifurcation

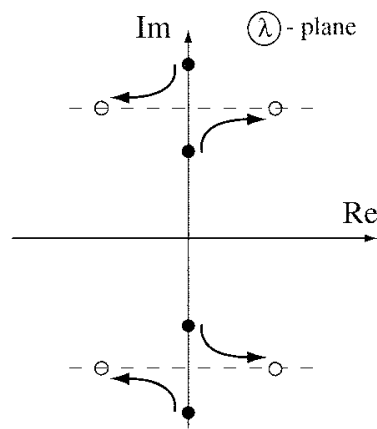

(b) $1: 1$ resonance (Hamiltonian Hopf) bifurcation
FIG. 14. Hamiltonian bifurcations: splitting of eigenvalues. Solid circles stand for an initial locus, while empty circles stand for the final state.

goal of this review is to understand the effect of dissipation, which leads to a structural change in the behavior of an originally conservative system, we first briefly review the state of affairs for bifurcations of Hamiltonian vector fields.

It is known that there are two kinds of generic local bifurcations from an equilibrium in Hamiltonian systems: (i) steady-state bifurcation when the linearized vector field at the equilibrium has a zero eigenvalue of multiplicity 2, and (ii) 1:1 resonance (codimension 1 bifurcation) when the linearization has a pair of purely imaginary eigenvalues of multiplicity 2 . In the case in which there are no symmetries present, the classification can be given based on the original work of Galin (1975), according to which in steady-state bifurcation the eigenspace has generic dimension, $\operatorname{dim} E=2$, and the corresponding normal form (result of versal deformations introduced by Poincaré or, which is the same, universal unfolding) of the linearized vector field,

$$
M(\lambda)=\left(\begin{array}{ll}
0 & 1 \\
\lambda & 0
\end{array}\right),
$$

so that as $\lambda$ increases through zero the eigenvalues $\pm \sqrt{\lambda}$ move along the imaginary axis and split onto the real axis, as shown in Fig. 14(a). In the case of the 1:1 resonance, the Galin normal form contains the block of $\operatorname{dim} E=4$,

$$
M(\lambda)=\left(\begin{array}{cccc}
0 & -1 & \rho & 0 \\
1 & 0 & 0 & \rho \\
\lambda & 0 & 0 & -1 \\
0 & 0 & 1 & 0
\end{array}\right), \quad \rho= \pm 1
$$

which exhibits the generic movement of eigenvalues, which first move along the imaginary axis and upon reaching 1:1 resonance split, i.e., move into the right and left halves of the complex plane, as shown in Fig. 14(b). This 1:1 resonance with splitting is often referred to as the Hamiltonian-Hopf bifurcation or Krein crash, since it was first accounted for by Daleckii and Krein (1974), who also provided a necessary condition for splitting
(Krein theorem). The above Galin forms indicate that splitting is generic in both the steady-state and 1:1 resonance bifurcations.

Example. As an illustration of the above discussion, consider a system with two degrees of freedom and the Hamiltonian

$$
\begin{aligned}
H= & \frac{1}{2 m}\left(p_{1}^{2}+p_{2}^{2}\right)+\omega_{G}\left(q_{2} p_{1}-q_{1} p_{2}\right) \\
& +\frac{1}{2}\left(\omega_{G}^{2}-\omega_{k}^{2}\right)\left(q_{1}^{2}+q_{2}^{2}\right),
\end{aligned}
$$

which has two time scales that are determined by two frequencies $\omega_{G}=G / m$ and $\omega_{k}=\sqrt{k / m}$, where $m$ stands for a mass, $k$ for a spring constant, and $G$ for a gyroscopic constant proportional to an angular speed of a rotating system. The corresponding Lagrangian that is related to $H$ by the inverse Legendre transform is $L$ $=\frac{1}{2} m\left(\dot{x}^{2}+\dot{y}^{2}\right)+G(\dot{y} x-\dot{x} y)+\frac{1}{2} k\left(x^{2}+y^{2}\right)$. The dynamics is apparently determined by the two characteristic exponents $\lambda= \pm i \omega_{k}\left(\epsilon \pm \sqrt{\epsilon^{2}-1}\right)$ and $\epsilon=\omega_{G} / \omega_{k}>0$. The parameter value $\epsilon=1$ corresponds to a 1:1 resonance and bifurcation from a stable situation (all eigenvalues are on the imaginary axis for $\epsilon \leqslant 1$ ) to an unstable case of complex eigenvalues $(\epsilon>1)$, as shown in Fig. 14(b).

The above example also demonstrates the concept of a negative energy mode (Morrison, 1998), which can be seen in the case $\epsilon>1$ after applying a canonical transformation $(\mathbf{q}, \mathbf{p}) \rightarrow(\mathbf{Q}, \mathbf{P})$, given by the generating function $F(\mathbf{q}, \mathbf{P})=c\left(q_{1} P_{s}+q_{2} P_{f}\right)+P_{f} P_{s}+\frac{1}{2} c^{2} q_{1} q_{2}$, where $c=\left[4\left(\omega_{G}^{2}\right.\right.$ $\left.\left.-\omega_{k}^{2}\right)\right]^{1 / 4}$ and $p_{i}=F_{q}{ }^{i}, \mathrm{Q}^{i}=F_{P_{i}}$ (Goldstein, 1956). This transformation takes the Hamiltonian $H(\mathbf{q}, \mathbf{p})$ into $H(\mathbf{Q}, \mathbf{P})=-\frac{1}{2} \omega_{s}\left(P_{s}^{2}+\mathrm{Q}_{s}^{2}\right)+\frac{1}{2} \omega_{f}\left(P_{f}^{2}+\mathrm{Q}_{f}^{2}\right)$, where $\omega_{f}=\omega_{G}+c^{2}$ and $\omega_{s}=\omega_{G}-c^{2}$. The latter is the linear part of Cherry's Hamiltonian (Cherry, 1925) and represents a sum of two oscillators, one slow and one fast. The slow oscillator is a negative energy mode: a decrease of the total energy $H(\mathbf{Q}, \mathbf{P})$ due to dissipation can be achieved by an increase of the amplitude of the slow mode, as can be observed from the above expression.

It is clear that the situations in Fig. 14 do not span all the possibilities and one can also expect that instead of splitting, the eigenvalues can pass, thus staying on the imaginary axis, as shown in Fig. 15. However, in the case in which the Hamiltonian system has no symmetries, at least three parameters are required for passing to be expected (Galin, 1975). However, eigenvalue passing is often encountered in applications due to the presence of various symmetries, which restrict the possible movements of eigenvalues. An understanding of the conditions for passing in the steady-state case was attained in the work of Golubitsky et al. (1987) and in the 1:1 resonance case in the work of van der Meer (1990), while the proofs have been given by Dellnitz et al. (1992). The latter work demonstrated that the dichotomy in eigenvalue movements can be understood only using both energetics and group theory (in the steady-state case, energetics suffices). While the presence of symmetries can restrict the eigenvalues to stay on the imaginary axis, the 


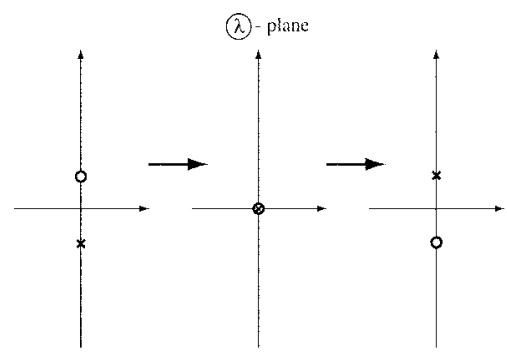

(a) Steady-state passing

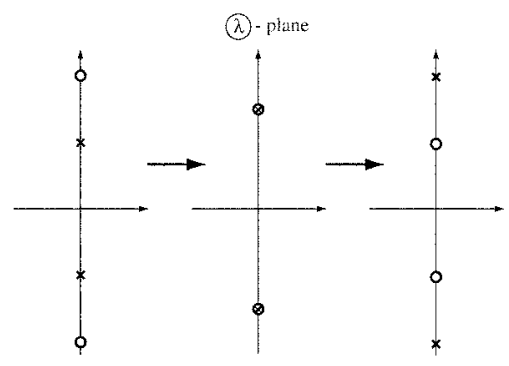

(b) 1:1 resonance passing

FIG. 15. Passing of eigenvalues in the Hamiltonian case.

same system but with different symmetries might have quite different stability characteristics, as was found by Guckenheimer and Mahalov (1992) [see also the work by Knobloch et al. (1994)]. They studied a system in $\mathbb{R}^{2}$, of the form $\dot{A}=i\left(\lambda A-|A|^{2} A-\epsilon \bar{A}\right)$ with $\lambda, \epsilon \in \mathbb{R}$, which possesses an $S^{1}$ symmetry group if the symmetry breaking parameter $\epsilon=0$, and $Z_{2}$ symmetry, given by the action $A \rightarrow-A$ in the complex plane, if $\epsilon \neq 0$. Because this system is only two dimensional, it is not amenable to dissipation-induced instabilities. Finally, in this context it is worth mentioning the work by Nagata and Namachchivaya (1998), who studied symmetry-breaking effects in gyroscopic systems in $\mathbb{R}^{4}$ in a rotating frame.

Summarizing, bifurcations of Hamiltonian vector fields include a steady state and a 1:1 resonance bifurcations from a stable equilibrium. Generically, this happens in systems without symmetries since in this case the eigenvalues split (which, however, may also take place when symmetries are present, though eigenvalue passing is more common in the latter case). In this context, it becomes clear that reduction of allowed symmetries may lead to destabilization, such as $S^{1} \rightarrow Z_{2}$ in the above discussed example of Guckenheimer and Mahalov (1992).

Concluding this discussion of Hamiltonian bifurcations, it is worth mentioning the case in which the system is spectrally stable, but in view of the presence of multiple eigenvalues and nontrivial Jordan block(s), the dynamics involves an algebraic growth and is thus unstable, as can be seen in the following trivial example: $\dot{q}=p$ and $\dot{p}=0$.

\section{B. Dissipation-induced movements of eigenvalues}

The goal of this subsection is to discuss two points of view on the subject, the classical one largely forgotten

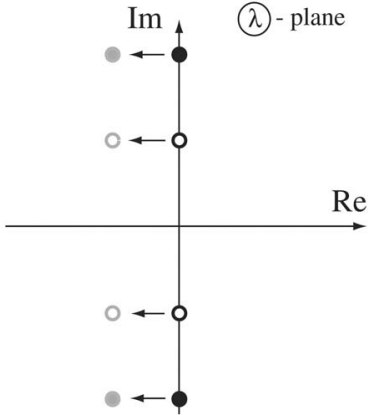

(a) Stabilization

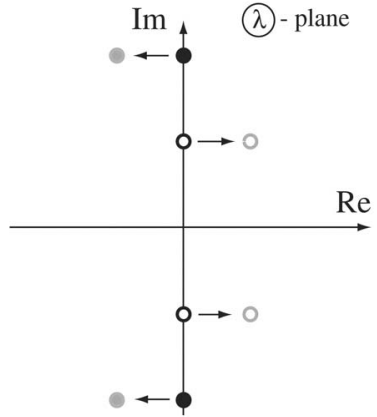

(b) De-stabilization
FIG. 16. Effects of dissipation on the stability of motion.

and the modern one developed in the context of bifurcations, and to show their overlap. To start with, consider the effect of dissipation on the 1:1 resonance, at which there are two alternative points of view. The first one comes from the work of Clerc and Marsden (2001), which claims that "... close to the 1:1 resonance, generically the dissipative terms induce an instability." However, there are many physical systems (and applications) when dissipation does not induce instability, as the following simple example indicates.

Example. Apparently, the following system with the 1:1 resonance remains stable after the addition of dissipation,

$$
\begin{aligned}
& \ddot{q}_{1}+d \dot{q}_{1}+c_{1} q_{1}=0, \\
& \ddot{q}_{2}+d \dot{q}_{2}+c_{2} q_{2}=0,
\end{aligned}
$$

where $d, c_{1}=c_{2}=c \in \mathbb{R}^{+}$. This system may come from a rotating shaft problem, for example. When dissipation is added, $d>0$, the eigenvalues move to the left of the imaginary axis. This behavior persists for the more general case, when there is no resonance, $c_{1} \neq c_{2}$, as in Fig. 16(a). The doubt in using "generically" comes from the fact that the cardinality of systems with stable potential energy is the same as the cardinality of systems with an unstable one. The presence of resonances is an equally rare phenomenon in both situations.

Based on the above observations, one can conclude that the 1:1 resonances and instabilities induced by dissipation are independent phenomena, but can overlap. The natural question is: Under which conditions can these phenomena overlap? We shall address it in this subsection. Let us consider the following simple system for $\mathbf{q}=\left(q_{1}, q_{2}\right)$ :

$$
\ddot{\mathbf{q}}+D \dot{\mathbf{q}}+G \dot{\mathbf{q}}+C \mathbf{q}=0
$$

with

$$
D=\left[\begin{array}{cc}
d_{1} & 0 \\
0 & d_{2}
\end{array}\right], \quad G=\left[\begin{array}{cc}
0 & g \\
-g & 0
\end{array}\right], \quad C=\left[\begin{array}{cc}
c_{1} & 0 \\
0 & c_{2}
\end{array}\right],
$$




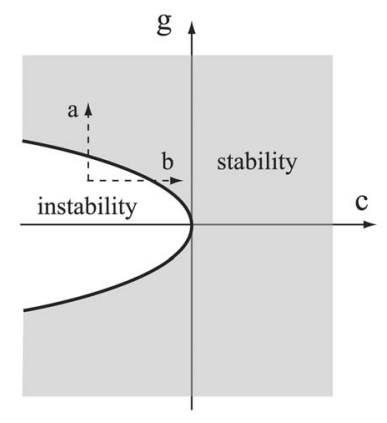

(a) No dissipation, $d=0$

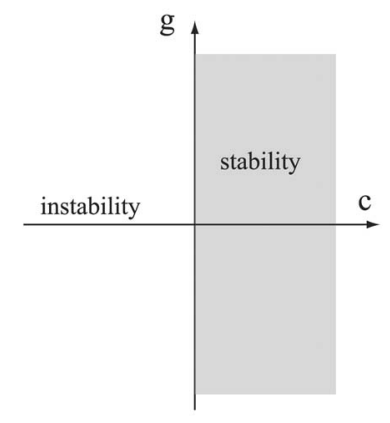

(b) With dissipation, $d>0$
FIG. 17. Effects of dissipation on the gyroscopic system (4.4).

which in the case $c_{1}=c_{2}=c$ and $d_{1}=d_{2}=d$ possesses $S^{1}$ symmetry, i.e., is equivariant with respect to the action $\mathbf{q} \rightarrow R_{\theta} \mathbf{q}$ for all $\theta \in S^{1}$, where $R_{\theta}$ is the rotation matrix,

$$
R_{\theta}=\left[\begin{array}{cc}
\cos \theta & \sin \theta \\
-\sin \theta & \cos \theta
\end{array}\right] \text {. }
$$

It is easy to determine that eigenvalues, as functions of the dissipation $d$, the gyroscopic parameter $g$, and potential energy effects measured by $c$, are given by

$$
\lambda_{1-4}=\frac{1}{2}\left[-(d \pm i g) \pm \sqrt{(d \pm i g)^{2}-4 c}\right],
$$

which, in the absence of dissipation $d=0$, have a $1: 1$ splitting bifurcation at $|g|=2 \sqrt{-c}$, as shown in Fig. 16(b). As allowed by the Thomson-Tait-Chetaev Theorem 4, if $c<0$, the system gets stabilized at a certain amplitude of gyroscopic force, $|g| \geqslant 2 \sqrt{-c}$, consistent with the condition that the degree of instability is even. This fact is reflected by the stability picture in the $(g, c)$ plane: both paths $a$ and $b$ shown in Fig. 17(a) lead to the 1:1 resonance. Once dissipation is added, $d>0$, the real part of the eigenvalues at small $d$ is given by $2 \operatorname{Re}(\lambda)$ $=-d \pm 2 d g / \sqrt{g^{2}+4 c}>0$, thus leading to instability for all $c<0$ in the $(g, c)$ plane [cf. Fig. 17(b)] by moving the eigenvalues off the imaginary axis in a fashion shown in Fig. 16(b) (this figure shows only one possibility, while the reversed direction of movement is also possible). It is notable that the complicated behavior of the eigenvalues discussed above is a simple consequence of algebra, ${ }^{12}$ since the eigenvalues (4.6) are solutions of the polynomial dispersion relation, i.e., the characteristic equation (4.4).

Concluding this section, the destabilizing effect of dissipation, as in Fig. 16(b), predicted by the Thomson-TaitChetayev Theorem is more robust and general than that restricted to the 1:1 resonance case, which is obviously just a particular situation, which might be of special in-

\footnotetext{
${ }^{12}$ The methods of algebra become useful if one wants to answer the questions like by which amount the eigenvalues move off the imaginary axis, which goes back to Daleckii and Krein (1974) [see also MacKay (1991)], and what is the number of eigenvalues in the right half plane usually dealt with the help of the Routh-Hurwitz criterion (Gantmacher, 1996).
}

terest in various applications once the nonlinearity is taken into account. ${ }^{13}$ From a stability standpoint, it follows that the 1:1 passing resonance is probably more interesting for the study of dissipative effects than the splitting case, since in the latter the instability develops in any event (if the Hamiltonian bifurcation parameter experiences an arbitrary small change) with or without dissipation.

So, what are the conditions (symmetries, energetics) that determine the effects capable of destabilizing the equilibria? The answer to this question can be seen with the help of the following two simple systems: a gyroscopically stabilized system with an unstable potential, i.e., $c<0$, to which the dissipative forces are applied,

$$
\ddot{\mathbf{q}}+\underline{D \dot{\mathbf{q}}}+G \dot{\mathbf{q}}+C \mathbf{q}=0,
$$

where

$$
D=d \text { id, } \quad C=c \text { id, } \quad G=\left[\begin{array}{cc}
0 & g \\
-g & 0
\end{array}\right],
$$

and a system with a stable potential, i.e., $c>0$, and under the action of positional forces,

$$
\ddot{\mathbf{q}}+C \mathbf{q}+\underline{P \mathbf{q}}=0, \quad C=c \text { id }, \quad P=\left[\begin{array}{cc}
0 & p \\
-p & 0
\end{array}\right] .
$$

When the underlined terms, i.e., destabilizing effects, are absent, both systems are stable and are in 1:1 resonance if $|g|=2 \sqrt{-c}$. Instability occurs if the nonzero underlined terms are added, no matter how small, in accordance with the corresponding Thomson-Tait-Chetayev and Merkin Theorems 3 and 5, respectively. Apparently, both systems possess an $S^{1}$ symmetry even if destabilizing effects are present. What is different though is the stability type of the potential energy surface (concave versus convex) and the destabilizing coupling (symmetric versus skew symmetric). Therefore, to isolate the most fundamental cause for this story, we can formulate the following theorem.

Theorem 6. Consider a Hamiltonian system, which has an equilibrium at $(0,0)$; assume it has a 1:1 resonance. Then, this equilibrium is destabilized (I) by arbitrarily small dissipative forces if the second variation $\left.\delta^{2} H\right|_{(0,0)}$ is indefinite, or (II) by arbitrarily small positional forces if $\left.\delta^{2} H\right|_{(0,0)}$ is definite.

The proof of the theorem is a straightforward consequence of Theorems 3 and 5. It would be interesting to identify these arbitrarily small destabilizing effects as the ones breaking some specific symmetry (other than $S^{1}$ and being non-Hamiltonian). While the distinction be-

\footnotetext{
${ }^{13}$ Physical situations, in which the $1: 1$ resonance is encountered, span from celestial mechanics (Szebehely, 1967) (e.g., the restricted three-body problem for the planar motion of a light body orbiting in the fields of two heavy bodies) and astrophysics (Kondrat'ev, 2000) (e.g., for star moving in a spheroidal, ring-shaped galaxy) to fluid dynamics [e.g., water waves (Buffoni and Groves, 1999)] and elastodynamics [e.g., flutter of a flag (Argentina and Mahadevan, 2005)].
} 
tween these two cases is still to be fully understood, in the next subsection we discuss a unified interpretation from the point of view of singularity theory. This interpretation also provides a geometric picture for the movement of eigenvalues.

\section{Connection to singularity theory}

The dichotomy of the eigenvalue movements studied above in the dissipative and conservative cases suggests that the Hamiltonian case represents some kind of singular limit (and highly degenerate, but nevertheless central, case) when viewed in the general class of dissipative systems. The singular limit here is understood in the context of stability: a stability is replaced by an instability if the bifurcation parameter experiences an arbitrarily small change from its zero value, which corresponds to no dissipation case. This raises the question of how reasonable it is to consider Hamiltonian models to account for real world behavior, in which dissipation, through interaction with unmodeled dynamics, is common. While there is no general answer to this question, we discuss one particular case, namely, the so-called Hamiltonian Hopf bifurcation, following the work of Langford (2003).

It is known that the Hamiltonian Hopf bifurcation is quite different from the classical Poincaré-Hopf bifurcation (Hopf, 1942), in which there are two complex conjugate eigenvalues (with nonzero frequencies) crossing the imaginary axis from left to right. In the Hamiltonian case the bifurcation occurs through eigenvalues splitting at the 1:1 resonance. Naturally, is it possible to characterize as a parametrized family all possible dissipative systems that are sufficiently close to the Hamiltonian Hopf bifurcation? The answer to this question can be given with the help of versal deformations following Arnold (1971), whose goal is to reduce a given family of matrices, which depend smoothly on the parameters, to the simplest form. In our case, the simplest form corresponds to the Hamiltonian Hopf bifurcation and we are looking for the family of vector fields that can be reduced to that form. Adopting complex variable notations as in Langford (2003), a linear two-dimensional Hamiltonian system, which undergoes a Hamiltonian Hopf bifurcation, can be transformed by a linear canonical transformation to the Jordan normal form

$$
\frac{\mathrm{d}}{\mathrm{d} t}\left[\begin{array}{l}
z_{1} \\
z_{2}
\end{array}\right]=\left[\begin{array}{ll}
i & 1 \\
0 & i
\end{array}\right]\left[\begin{array}{l}
z_{1} \\
z_{2}
\end{array}\right] .
$$

This system possesses the multiple eigenvalue $\lambda=i$, and together with its complex conjugate yields the standard picture of the 1:1 resonance. The symplectic matrix in Eq. (4.9) is the Jordan canonical form of a family of matrices that form a codimension-2 submanifold of $\mathrm{C}^{4}$, i.e., a versal deformation has at least two (complex) parameters. This is contrary to the symplectic matrix $\left[\begin{array}{cc}i & 0 \\ 0 & i\end{array}\right]$, the versal deformation of which has four parameters (matrices having this Jordan normal form are a codimension-4 submanifold of $\mathrm{C}^{4}$ ) (Wiggins, 2003). Ver-

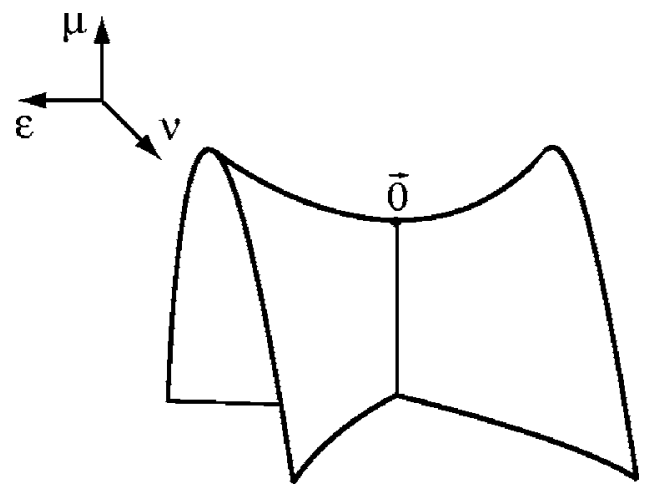

FIG. 18. Whitney umbrella (4.11).

sal deformations produce a three-parameter (real unfolding parameter) family of nonconservative systems of the form

$$
\frac{\mathrm{d}}{\mathrm{d} t}\left[\begin{array}{l}
z_{1} \\
z_{2}
\end{array}\right]=\left[\begin{array}{cc}
i+\epsilon & 1 \\
\mu+i \nu & i+\epsilon
\end{array}\right]\left[\begin{array}{l}
z_{1} \\
z_{2}
\end{array}\right],
$$

with the corresponding eigenvalues $\lambda_{ \pm}=(i+\epsilon) \pm \sqrt{\mu+i \nu}$. The necessary condition for the classical Hopf bifurcation is

$$
\operatorname{Re}\left(\lambda_{ \pm}\right)=0: \nu^{2}+4 \epsilon^{2}\left(\mu-\epsilon^{2}\right)=0,
$$

which defines a topology of the so-called Whitney umbrella $^{14}$ (Whitney, 1943) in the $(\nu, \mu, \epsilon)$-parameter space, as shown in Fig. 18. The umbrella self-intersects transversely along the $\mu$ axis for $\mu<0$ with crossing angle at the intersection going to zero as $\mu \rightarrow-0$. Whitney umbrella may be pictured as a self-intersecting rectangle in three dimensions. It possesses a pinch point, which occurs at the top end point of the segment of self-intersection. In every neighborhood of the pinch point, the surface intersects itself. Pinch points are also called Whitney singularities or branch points.

At every point of the umbrella, except for the points on the $\mu$ axis, there is one purely imaginary eigenvalue and one with nonzero real part. On the negative part of the $\mu$ axis, there are two distinct purely imaginary eigenvalues, which coalesce as double purely imaginary eigenvalues at the origin of Fig. 18. Finally, on the positive part of the $\mu$ axis, the four eigenvalues are symmetrically arranged as in the final state in Fig. 14(b). Therefore, the $\mu$ axis corresponds to the Hamiltonian case and eigenvalue movement as in Fig. 14(b). The umbrella divides $\mathbb{R}^{3}$ into three disjoint open regions, in each of which the real parts of the eigenvalues have distinct behavior, namely, $\epsilon>0: \operatorname{Re}\left(\lambda_{ \pm}\right)>0, \epsilon<0: \operatorname{Re}\left(\lambda_{ \pm}\right)<0$; above the umbrella the real parts have opposite signs. Concluding, the codimension-1 Hamiltonian Hopf bifurcation is a singular limit of the codimension-3 dissipative normal form.

\footnotetext{
${ }^{14}$ The canonical form of the Whitney umbrella is given by $y^{2}=z x^{2}$.
} 


\section{Summary}

In this section, the manifestation of dissipationinduced instabilities in terms of eigenvalue movement was discussed. In particular, the interaction of dissipative effects with the behavior of eigenvalues at a 1:1 resonance, and in Theorem 6 identified the conditions under which an instability occurs was explored. Next, the effect of dissipation on the movement of eigenvalues in the nonresonant case as well was discussed. Finally, a connection to singularity theory was established by investigating the transition from the Poincaré-Hopf to the Hamiltonian-Hopf bifurcations using versal deformations. It is important to stress that there are physical systems in which implications of singularity theory are not fully understood yet: one of them-a double spherical pendulum, which may experience a Hamiltonian Hopf bifurcation (Marsden and Scheurle, 1993)—will be discussed in the next sections, but in different contexts, namely, relative equilibria in Sec. $\mathrm{V}$ and control in Sec. VI.

\section{DISSIPATION-INDUCED INSTABILITIES OF RELATIVE EQUILIBRIA}

The natural question one might ask is: What would be the effect of dissipation if the equilibria is not ordinary, but relative? As we observed on the example of a rolling disk in Sec. III.A, the answer to this question can be reduced to studying the effect of dissipation on ordinary equilibria. The starting point in developing a general methodology is to realize that if we want to study the stability of relative equilibria in the Hamiltonian case, then we are dealing with Hamiltonian systems, which are symmetric, i.e., invariant under the action of a group $G$.

At first, a few epistemological remarks are provided. It appears that the study of the destabilizing effect of dissipation on Hamiltonian systems was recently continued in the works of Bloch et al. $(1994,1996)$ and Derks and Ratiu (2002), but the contribution of these works is limited to the case in which addition of dissipation to a Hamiltonian system preserves the symmetry related to the relative equilibria. The first work (Bloch et al., 1994) proves a dissipation-induced instability when dissipation does not destroy the conservation law associated with the symmetry group. The work of Derks and Ratiu (2002) relaxes this assumption by studying the case of invariant manifolds of relative equilibria when dissipation leaves the family of relative equilibria invariant, which the orbits of individual relative equilibria do not have to be invariant. The study of Bloch et al. (1996), which is a sequel of Bloch et al. (1994), considers the dissipation terms in Brockett's double bracket form, which are encountered, for example, in the dissipative mechanisms in ferromagnetism. The conclusion of that work similarly indicates that the addition of dissipative effects to a formally unstable equilibrium leads to instability.

The discussion in this section is on simple mechanical systems (i.e., for which the Hamiltonian is separable) only, while the generalizations of some of the ideasnamely, the reduction procedure-to natural mechanical systems (i.e., when the Hamiltonian is nonseparable), can be found in Lewis (1992) and Wang and Krishnaprasad (1992).

\section{A. The concept of relative equilibria and the history of reduction}

In analyzing dissipation-induced instabilities, we naturally start the discussion with Hamiltonian systems, which, in addition to being conservative, may also be invariant under the action of a continuous symmetry group. A natural class of solutions of such Hamiltonian systems are solutions moving with the flow of the symmetry group, i.e., whose dynamic orbit coincides with a one-parameter group orbit, and includes relative equilibria that were known at least to Routh and Poincaré, who realized the necessity of distinguishing this type of equilibria. For instance, if the symmetry group is a rotation group, then the relative equilibria is a uniformly rotating state: e.g., the circular orbit of a geostationary satellite or the rotation of a flywheel about its axis of symmetry. In fields other than mechanics, relative equilibria may have other names, e.g., rotating waves or simply basic states in fluid mechanics.

The presence of symmetry implies an existence of conservation laws (constants of motion) other than the Hamiltonian, as follows from Noether's theorem. Correspondingly, relative equilibria in Hamiltonian systems are critical points of the Hamiltonian constrained to the constants of motion related to the symmetries. The existence of relative equilibria is contingent on the presence of symmetries: once the symmetry is broken, the relative equilibrium solution disappears. The relative equilibrium also becomes an ordinary equilibrium once the problem is transformed to a frame that "sticks" with the relative equilibrium solution; for example, if the relative equilibrium is a uniformly rotating state, then it becomes an ordinary equilibrium in a coordinate system uniformly rotating with the same rate as the relative equilibrium. It is clear that, as a result of this procedure, the linear operator, which is important for stability analysis, might become time dependent, that is, with variable coefficients, and thus the classical ThomsonTait-Chetayev Theorem is not applicable. There are numerous examples in fluid dynamics when the nontrivial basic state resulting from the presence of symmetries leads to an inhomogeneous linearized operator. However, there are many examples when those theorems and/or analogous analyses are useful: one of them was the example on stability of a disk rolling along a straight line in Sec. III.A. Moreover, in contrast to the transformation to a rotating frame of reference, the reduction procedures, including the one described in this section, in these situations lead to time-independent operators on the reduced space.

Thus, in studying relative equilibria, a natural step involves the reduction of the original system. In the mid- 
1800 s, Routh was interested in rotating mechanical systems, such as those possessing an angular momentum conservation law. In this context, Routh used the term steady motion for dynamic motions that were uniform rotations about a fixed axis. According to modern understanding, these motions are ordinary equilibria of the reduced equations and relative equilibria of the EulerLagrange system before reduction. It was Poincaré who introduced this clarification around 1890. First, start with the notion of relative equilibria for a general dynamical system $\dot{\mathbf{x}}=f(\mathbf{x})$. Let $G$ be a compact Lie group acting orthogonally on $\mathbb{R}^{n}$, and let $f(\mathbf{x}): \mathbb{R}^{n} \rightarrow \mathbb{R}^{n}$ be a $G$-equivariant vector field. A group orbit $X$ is a relative equilibrium if the flow of the dynamical system leaves $X$ invariant (alternatively, $X$ is a relative equilibrium if $f$ is tangent to $X$ at points of $X$ ).

In the Hamiltonian context, consider a finitedimensional symplectic manifold $(M, \omega)$, where $\omega$ is a symplectic form on the manifold $M$. Suppose on $(M, \omega)$ we have a symmetric Hamiltonian system $\dot{\mathbf{x}}=X_{H}(\mathbf{x}, t)$, where $X_{H}(\mathbf{x}, t)$ is a vector field on $M$ that defines a flow $\phi_{t}(\mathbf{x}(0))=\mathbf{x}(t)$, with a Lie group $G$ acting on $M$ and $G$-invariant Hamiltonian $H$. The Lie algebra of the group $G$ is denoted by $\mathfrak{g}$. A point $z_{e}$ in the phase space $P$ is a relative equilibrium if the Hamiltonian vector field $X_{H}\left(z_{e}\right)$ points in the direction of the group orbit through $z_{e}$ :

Definition 4. A point $z_{e} \in P$ is called a relative equilibrium if $X_{H}\left(z_{e}\right) \in T_{z_{e}}\left(G z_{e}\right)$, that is, if the Hamiltonian vector field $X_{H}$ at $z_{e}$ points in the direction of the group orbit.

Equivalently, a point $\mathbf{m} \in M$ is a relative equilibrium of an invariant Hamiltonian if there exists a $\xi \in \mathfrak{g}$ such that $e^{t \xi} \mathbf{m}$ is a solution of the Hamiltonian system, i.e., $\phi_{t}(\mathbf{m})=e^{t \xi} \mathbf{m}$. Here $e^{t \xi}$ is a group flow generated by the element of Lie algebra $\xi \in \mathfrak{g}$.

In the Lagrangian context, consider the EulerLagrange equations,

$$
\frac{\mathrm{d}}{\mathrm{d} t} \frac{\partial L}{\partial \dot{q}^{j}}-\frac{\partial L}{\partial q^{j}}=0, \quad j=1, \ldots, n .
$$

Let, as usual, $p_{j}=\partial L / \partial \dot{q}^{j}$ be a generalized (canonical, conjugate) momentum. Assume that there are cyclic (ignorable) coordinates $q^{j}$, which by definition correspond to $\partial L / \partial q^{j}=0$, so that the corresponding conjugate momenta are conserved $\dot{p}_{j}=0$, i.e., $p_{j}=$ const. Therefore, in the Hamiltonian description used here there are fewer variables to solve for. The latter usually serves as a motivation for an introduction of the Routh procedure, an exposition of which may be found in Goldstein (1956). The same point of view goes back to Whittaker (1917), who explicitly stated that the Routh procedure is a special case of Hamiltonian transformation. However, it should be kept in mind that this classical way of motivating the Routh reduction can be misleading, since it does not allow one to understand the reduction in the non-Abelian case. The latter issue was understood and led to the extension of the classical Routh procedure to the non-Abelian case by Marsden and Scheurle (1993). It should be mentioned that the original reasoning by Routh (1913) was based on a simple observation that Eq. (5.1) simplifies for each cyclic (or "absent") coordinates $q^{j}$, since $\partial L / \partial q^{j}=0$.

In nonintrinsic terms, the essence of the Routh procedure in the classical case is to transform the cyclic coordinates, say with indexes $i=1, \ldots, s$, to the Hamiltonian formulation by performing a partial Legendre transform (fiber derivative) $\mathbb{F} L: T \mathrm{Q} \rightarrow T^{*} \mathrm{Q}$ (in coordinates $q^{i}, \dot{q}^{j}$ $\left.\rightarrow q^{i}, p_{j}\right)^{15}$ as applied to $H(\mathbf{q}, \mathbf{p}, t)=\sum \dot{q}^{i} p_{i}-\left.L(\mathbf{q}, \dot{\mathbf{q}}, t)\right|_{\dot{\mathbf{q}} \rightarrow \mathbf{p}}$, while the rest of the coordinates, $i=s+1, \ldots, n$, live in the Lagrangian frame. As a result, one gets

$$
\begin{aligned}
& \frac{\partial R}{\partial p_{i}}=\dot{q}^{i}, \quad \frac{\partial R}{\partial q^{i}}=-\dot{p}_{i}, \quad i=1, \ldots, s, \\
& \frac{\mathrm{d}}{\mathrm{d} t} \frac{\partial R}{\partial \dot{q}^{i}}-\frac{\partial R}{\partial q^{i}}=0, \quad i=s+1, \ldots, n,
\end{aligned}
$$

where

$$
R\left(q_{1}, \ldots, q^{n}, p_{1}, \ldots, p_{s}, \dot{q}^{s+1}, \ldots, \dot{q}^{n}, t\right)=\sum_{i=1}^{s} p_{i} \dot{q}^{i}-L .
$$

This again explains why the Routh method in the case of Abelian symmetries can be understood as having a foot in both the Lagrangian (5.3) and Hamiltonian (5.2) formulations. Equations (5.3) are reduced Euler-Lagrange equations.

As an illustration, consider a natural mechanical system on the configuration manifold $\mathrm{Q}=S \times\left(S^{1} \times \cdots\right.$ $\left.\times S^{1}\right)$ with coordinates $\left(x^{1}, \ldots, x^{m}\right)$ on the shape space $S$ and cyclic coordinates $\left(\theta^{1}, \ldots, \theta^{k}\right)$ on factors $S^{1}$ (the symmetry group $G=S^{1} \times \cdots \times S^{1}$ is Abelian), for which the Lagrangian has the form kinetic minus potential energy,

$$
\begin{aligned}
L(x, \dot{x}, \dot{\theta})= & \frac{1}{2} g_{\alpha \beta}(x) \dot{x}^{\alpha} \dot{x}^{\beta}+g_{a \alpha}(x) \dot{x}^{\alpha} \dot{\theta}^{a} \\
& +\frac{1}{2} g_{a b}(x) \dot{\theta}^{a} \dot{\theta}^{b}-V(x) .
\end{aligned}
$$

Because $\theta^{a}$ are cyclic, the corresponding conjugate momenta $p_{a}=\partial L / \partial \dot{\theta}^{a}=g_{a \alpha} \dot{x}^{\alpha}+g_{a b} \dot{\theta}^{b} \equiv \mu_{a}$ are conserved quantities, $\mu_{a}=$ const. The corresponding Routhian is

$$
\begin{aligned}
R^{\mu}(x, \dot{x})= & g_{a \alpha} g^{a c} \mu_{c} \dot{x}^{\alpha}+\frac{1}{2}\left(g_{\alpha \beta}-g_{a \alpha} g^{a c} g_{c \beta}\right) \dot{x}^{\alpha} \dot{x}^{\beta} \\
& -V_{\mu}(x),
\end{aligned}
$$

where $V_{\mu}=V(x)+\frac{1}{2} g^{a b} \mu_{a} \mu_{b}$ is the amended potential and $g^{a b}$ are entries of the inverse matrix of $g_{a b}$. It is notable that, due to the reduction, both the potential and kinetic energies are changed, and the Routhian $R^{\mu}$ has picked up a term linear in the velocity, which has a meaning of an extra force having a structure of a Coriolis force in this case. The positive definite nature of the kinetic energy term in $R^{\mu}$ can be seen by rewriting it in a form

\footnotetext{
${ }^{15}$ Note that one can solve $p_{i}=\partial L / \partial \dot{q}^{i}$ for $\dot{\mathbf{q}}$ locally only if Hes$\operatorname{sian} \operatorname{det}\left(L_{\dot{\mathbf{q}} \dot{\mathbf{q}}}^{\prime \prime}\right) \neq 0 ; \mathbb{F} L$ is a diffeomorphism if $L$ is hyper-regular.
} 


$$
\frac{1}{2}\left[\dot{x}^{\alpha},-A_{\delta}^{a} \dot{x}^{\delta}\right]\left[\begin{array}{ll}
g_{\alpha \beta} & g_{\alpha b} \\
g_{a \beta} & g_{a b}
\end{array}\right]\left[\begin{array}{c}
\dot{x}^{\beta} \\
-A_{\gamma}^{b} \dot{x}^{\gamma}
\end{array}\right],
$$

where $A_{\alpha}^{a}=g^{a b} g_{b \alpha}$. In the above illustration, we took the coordinate viewpoint, but it should be kept in mind that this cannot be done globally in general; instead, the intrinsic approach should be taken, as in the case of a double spherical pendulum (Marsden and Scheurle, 1993).

As noted by Arnold (1993), the problem of reduction is linked to the question of hidden motions, which troubled physicists at the end of the 19th century: Helmholtz, J. J. Thomson, and Hertz insisted that every mechanical quantity, which manifests itself as a potential energy (i.e., does not depend on velocities), is in fact the kinetic energy of hidden motions under which only cyclic (hidden) coordinates vary. A typical example is the rotation of a symmetric top: we may perceive that the top does not rotate and explain its behavior and stability as if it is acted upon by some conservative forces. Finally, the Routh reduction example is the elimination of the polar angle in Kepler's problem presented below for illustration of the above exposition and for the needs of further discussion.

Example. Consider the classical two-body (Kepler's) problem (Goldstein, 1956; Smale, 1970; Arnold, 1993) of the motion of two bodies of mass $m_{1}$ and $m_{2}$, respectively, in the potential field $U=U(|\mathbf{r}|), \mathbf{r}=\mathbf{r}_{1}-\mathbf{r}_{2}$,

$$
m_{1} \ddot{r}_{1}=-\frac{\partial U}{\partial r_{1}}, \quad m_{2} \ddot{r}_{2}=-\frac{\partial U}{\partial r_{2}} .
$$

Since in a barycentric coordinate system the trajectories of the two point masses are similar planar curves (with similarity ratio $m_{1} / m_{2}$ ), then the problem reduces to the investigation of a single equation, namely,

$$
m \ddot{r}=-\frac{\partial U}{\partial r},
$$

for $m=m_{1} m_{2} /\left(m_{1}+m_{2}\right)$. The Lagrangian of this system in polar coordinates is

$$
L=T-U=\frac{m}{2}\left(\dot{r}^{2}+r^{2} \dot{\theta}^{2}\right)-U(r),
$$

so that the coordinate $\theta$ is obviously cyclic. The conserved momentum is $p_{\theta}=m r^{2} \dot{\theta} \equiv l$, so that the total energy can be written as

$$
E=T+V=\frac{m}{2} \dot{r}^{2}+\frac{1}{2} \frac{l^{2}}{m r^{2}}+V(r),
$$

and Eq. (5.8) reduces to

$$
m \ddot{r}=-\frac{\partial U_{c}}{\partial r},
$$

where $U_{c}=\frac{1}{2}\left(l^{2} / m r^{2}\right)+V(r)$ is the reduced (also known as amended or fictitious) potential, i.e., the potential function amended by adding a term corresponding to a "centrifugal force." It is notable that the usual potential $U$

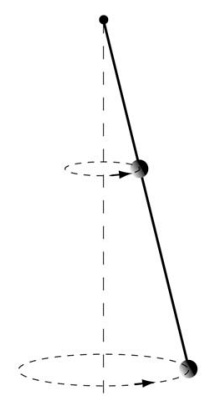

(a) Stretched out solution

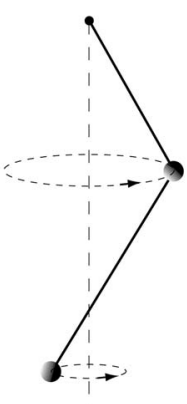

(b) Cowboy solution
FIG. 19. Two relative equilibria of the double spherical pendulum.

has no extrema, while the amended one has a minimum (potential well), which explains the orbital stability.

It fact, any generic two-dimensional gyroscopically stabilized system is closely analogous to the Kepler problem, but with a different potential function in general, e.g., the restricted three-body problem (Murray, 1994), a planar oscillator on a rotating plate, and the charged spherical pendulum (Bloch et al., 2004); see also the discussion in Sec. III.A.

\section{B. Cotangent bundle reduction}

To illustrate the effect of dissipation on relative equilibria, we consider the case of a double spherical pendulum, shown in Fig. 19. First we introduce the necessary elements of the cotangent bundle reduction.

Consider an abstract mechanical system on a configuration manifold $\mathrm{Q}$, and canonical phase space $P$, which is the cotangent bundle $P=T^{*} \mathrm{Q}$. Assume that the mechanical system is Hamiltonian, with the Hamiltonian function denoted by $H: P \rightarrow \mathbb{R}$, which represents the total energy of the system. We denote by $q$ an element of $\mathrm{Q}$, and say that coordinates $q^{i}$ on $\mathrm{Q}$ induce coordinates $\left(q^{i}, p_{i}\right)$ on $T_{q}^{*} \mathrm{Q}$, where $\mathbf{p} \in T_{q}^{*} \mathrm{Q}$ is the associated momentum. ${ }^{16}$ The pair $(\mathbf{q}, \mathbf{p}) \in \mathrm{Q} \times T_{q}^{*} \mathrm{Q}$ of the canonical cotangent coordinates is identified with $z \in T^{*} \mathrm{Q}$. Further, assume that the Hamiltonian system possesses symmetry induced by a Lie group $G$ with a Lie algebra $\mathfrak{g}$. Associated to the action of $G$ on $\mathrm{Q}$ are the infinitesimal generators $\zeta_{\mathrm{Q}}(q)$, which form the tangent space to the group orbit $G q$.

By Noether's theorem, for each continuous symmetry $\xi \in \mathfrak{g}$ there is a conserved quantity $\mathbf{J}(\xi)$ that has the same dimension as the group $G$ has. If $\mathfrak{g}^{*}$ is the dual of the Lie algebra $\mathfrak{g}$, then, as a generalization of linear or angular momenta, we introduce a momentum map $\mathbf{J}: P \rightarrow \mathfrak{g}^{*}$ for the action of $G$ on $P=T^{*} \mathrm{Q}$, which reproduces as special cases the usual angular and linear momenta. The function $\mathbf{J}$ is determined by

\footnotetext{
${ }^{16}$ The tangent spaces $T_{q} \mathrm{Q}$ and $T_{q}^{*} \mathrm{Q}$ are in natural duality via the nondegenerate pairing $\langle\cdot, \cdot\rangle$.
} 


$$
\mathbf{J}(z) \cdot \xi=\left\langle\mathbf{p}, \xi_{\mathrm{Q}}(q)\right\rangle, \quad \mathbf{p} \in T_{q}^{*} \mathrm{Q}
$$

for all $\xi \in \mathfrak{g}$. Let $\left\{e_{1}, \ldots, e_{n}\right\}$ be the basis for $\mathfrak{g}_{\mu}$, which is the Lie algebra of $G_{\mu}$; then for $\xi \in \mathfrak{g}_{\mu}$ we have $\xi=\xi^{j} e_{j}$. If in coordinates the infinitesimal generator is $\xi_{\mathrm{Q}}^{i}(q)$ $=K_{a}^{i}(q) \xi^{a}$ (here $\xi^{a}$ are components on $\mathfrak{g}$ ), then in canonical coordinates $p_{q}=\left(q^{i}, p_{i}\right)$ we get $\langle\mathbf{J}, \xi\rangle\left(p_{q}\right)=p_{i} K_{a}^{i}(q) \xi^{a}$, i.e., $J_{a}=p_{i} K_{a}^{i}(q)$. Here $K_{a}^{i}$ is called the action tensor.

Next, consider the diagram

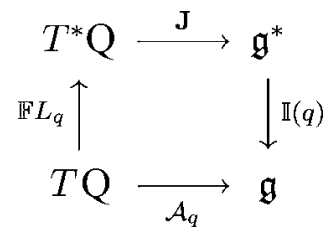

where coordinates are $(q, p) \in T^{*} \mathrm{Q}$ and $(q, v) \in T \mathrm{Q}$. We use shorthand $p_{q}$ and $v_{q}$. We are left to define $\mathbb{I}(q)$ and $\mathcal{A}_{q}$ in this diagram to have a complete geometric characterization of the cotangent bundle reduction.

Definition 5. For each $q \in \mathrm{Q}$, the locked inertia tensor is the isomorphism $\mathbb{I}(q): \mathfrak{g}_{\mu} \mapsto \mathfrak{g}_{\mu}^{*}$ given by

$$
\mathbb{I}(q) \eta \xi=\left\langle\eta_{\mathrm{Q}}(q), \xi_{\mathrm{Q}}(q)\right\rangle
$$

for $\xi, \eta \in \mathfrak{g}_{\mu}$.

Since the action of $G$ on $\mathrm{Q}$ is free, $\mathrm{I}(q)$ is an inner product (Riemannian metric). This terminology comes from the fact that for coupled rigid or elastic systems $\mathrm{I}(q)$ is the classical moment of inertia tensor of the rigid body, obtained by locking all the joints of the system. In coordinates, for simple mechanical system $H(q, p)$ $=\frac{1}{2} g^{i j} p_{i} p_{j}+V(q)$, we have

$$
\mathrm{I}_{i j}(q)=g_{k l}(q) K_{i}^{k}(q) K_{j}^{l}(q) \text {. }
$$

To close the "loop" in Eq. (5.13), we define the connection one-form for the mechanical connection: $\mathcal{A}_{q}: T \mathrm{Q} \rightarrow \mathfrak{g}$, which is actually an angular velocity of the locked system. It is a $\mathfrak{g}$-valued one-form on $\mathrm{Q}$ and defined by

$$
\begin{aligned}
& \mathcal{A}(q, v)=\mathbb{I}^{-1}(q)[\mathbf{J}(\mathbb{F} L(q, v))], \\
& \text { that is, } \mathcal{A}^{i}\left(v_{q}\right)=\mathbb{I}^{i j}(q) g_{k l}(q) K_{j}^{k}(q) v^{l},
\end{aligned}
$$

where $\mathbb{I}^{i j}(q)$ are components of the inverse of $\mathbb{I}_{i j}(q)$. Note that $\mathcal{A}(q, v)$ has the meaning of the angular velocity of the locked system, and $\mathbb{F} L: T \mathrm{Q} \rightarrow T^{*} \mathrm{Q}$ is the metric tensor regarded as a map from vectors to covectors, $\mathbb{F} L(q, v)=(q, p)$, where $p_{i}=g_{i j} v^{j}$. One should also think of $\mathcal{A}$ as a connection on the principal $G$ bundle $\mathrm{Q} \rightarrow \mathrm{Q} / G$, i.e., $\mathcal{A}$ is $G$ invariant and satisfies $\mathcal{A}\left(\xi_{\mathrm{Q}}(q)\right)=\xi$. Classically, a connection (Arnold, 1993) is an invariant splitting of the tangent space $T_{q} \mathrm{Q}$ into horizontal and vertical vectors, i.e., the distribution of horizontal vectors is invariant under the action of $G$. The basic idea behind this is very simple: the larger space is projected on the smaller space-base space; directions in the larger space that project to zero are vertical; the connection is a specification of the horizontal directions, which complement the space of vertical directions. The horizontal space hor $q$ of the connection $\mathcal{A}$ at $q \in \mathrm{Q}$ is given by the kernel of $\mathcal{A}_{q}$,

$$
\operatorname{hor}_{q}=\{(q, v) \mid \mathbf{J}(\mathbb{F} L(q, v))=0\} .
$$

Since $\mathbf{J}(z) \cdot \xi=\left\langle\mathbf{p}, \xi_{\mathrm{Q}}(q)\right\rangle$, we see that hor $_{q}$ is the space orthogonal to the $G$ orbits. On the contrary, the vertical space consists of vectors that are mapped to zero under the projection $\mathrm{Q} \rightarrow S=\mathrm{Q} / G$, i.e., $\operatorname{ver}_{q}=\left\{\xi_{\mathrm{Q}}(q) \mid \xi \in \mathfrak{g}\right\}$. Thus, the horizontal-vertical decomposition of a vector $(q, v) \in T_{q} \mathrm{Q}$ is just $v=\operatorname{hor}_{q} v+\operatorname{ver}_{q} v$. For each $\mu \in \mathfrak{g}^{*}$, define the 1 -form $\mathcal{A}_{\mu}$ on $\mathrm{Q}$ by $\left\langle\mathcal{A}_{\mu}(q), v\right\rangle=\langle\mu, \mathcal{A}(q, v)\rangle$, that is, $\left(\mathcal{A}_{\mu}\right)_{i}=g_{i j} K_{b}^{j} \mu_{a} \rrbracket^{a b}$.

Having defined all geometric structures (5.13) pertinent to the cotangent bundle reduction, we formulate the main outcome of this reduction, which is actually a generalization of the ideas introduced in Sec. V.A and Kepler's example.

Definition 6. The amended potential $V_{\mu}$ is defined by $V_{\mu}=H \circ \mathcal{A}_{\mu}$, i.e., intrinsically $V_{\mu}(q)=V(q)+\frac{1}{2}\left\langle\mu, \mathbb{I}^{-1}(q) \mu\right\rangle$, or in coordinates $V_{\mu}(q)=V(q)+\frac{1}{2} \coprod^{a b}(q) \mu_{a} \mu_{b}$.

Example (double spherical pendulum, Fig. 19). To explore the above ideas, we use, as an illustration, the mechanical system consisting of two coupled spherical pendula in a gravitational field following the discussions by Marsden (1992) and Marsden and Scheurle (1993). The configuration space is $\mathrm{Q}=S_{1}^{2} \times S_{2}^{2}$, i.e., the product of two spheres of radii $l_{1}$ and $l_{2}$, respectively, and corresponding coordinates $\mathbf{q}_{1}$ and $\mathbf{q}_{2}$. The respective Lagrangian is of the form appropriate for simple mechanical systems, i.e., kinetic minus potential energies,

$$
\begin{aligned}
L\left(\mathbf{q}_{1}, \mathbf{q}_{2}, \dot{\mathbf{q}}_{1}, \dot{\mathbf{q}}_{2}\right)= & \frac{m_{1}}{2}\left\|\dot{\mathbf{q}}_{1}\right\|^{2}+\frac{m_{2}}{2}\left\|\dot{\mathbf{q}}_{1}+\dot{\mathbf{q}}_{2}\right\|^{2}-m_{1} g \mathbf{q}_{1} \cdot \mathbf{k} \\
& -m_{2} g\left(\mathbf{q}_{1}+\mathbf{q}_{2}\right) \cdot \mathbf{k},
\end{aligned}
$$

from where we obtain the cotangent bundle $T^{*} \mathrm{Q}$, with conjugate momenta $\mathbf{p}_{1}=L_{\dot{\mathbf{q}}_{1}}=m_{1} \dot{\mathbf{q}}_{1}+m_{2}\left(\dot{\mathbf{q}}_{1}+\dot{\mathbf{q}}_{2}\right)$ and $\mathbf{p}_{2}$ $=L_{\dot{\mathbf{q}}_{2}}=m_{2}\left(\dot{\mathbf{q}}_{1}+\dot{\mathbf{q}}_{2}\right)$, and the Hamiltonian

$$
\begin{aligned}
H\left(\mathbf{q}_{1}, \mathbf{q}_{2}, \mathbf{p}_{1}, \mathbf{p}_{2}\right)= & \frac{\left\|\mathbf{p}_{1}-\mathbf{p}_{2}\right\|^{2}}{2 m_{1}}+\frac{\left\|\mathbf{p}_{2}\right\|^{2}}{2 m_{2}}+m_{1} g \mathbf{q}_{1} \cdot \mathbf{k} \\
& +m_{2} g\left(\mathbf{q}_{1}+\mathbf{q}_{2}\right) \cdot \mathbf{k} .
\end{aligned}
$$

The continuous symmetry group is simply a simultaneous rotation about the $z$ axis, $G=S^{1}$, i.e., the group action is $\left(\mathbf{q}_{1}, \mathbf{q}_{2}\right) \rightarrow\left(R_{\theta} \mathbf{q}_{1}, R_{\theta} \mathbf{q}_{2}\right)$, where $R_{\theta}$ is the rotation by an angle $\theta$. The element of the Lie algebra is the rotation vector $\xi=\omega \mathbf{k} \in \mathfrak{g} \simeq \mathbb{R}$ with the infinitesimal generator $\omega\left(\mathbf{k} \times \mathbf{q}_{1}, \mathbf{k} \times \mathbf{q}_{2}\right)$ and thus the momentum map is

$$
\langle\mathbf{J}, \omega \mathbf{k}\rangle=\omega\left[\mathbf{p}_{1} \cdot\left(\mathbf{k} \times \mathbf{q}_{1}\right)+\mathbf{p}_{2} \cdot\left(\mathbf{k} \times \mathbf{q}_{2}\right)\right],
$$

i.e., $\mathbf{J}=\mathbf{k} \cdot\left[\mathbf{q}_{1} \times \mathbf{p}_{1}+\mathbf{q}_{2} \times \mathbf{p}_{2}\right]$. The locked inertia tensor is found by identifying the metric in Eq. (5.18),

$$
\begin{aligned}
& \left\langle\mathbb{I}\left(\mathbf{q}_{1}, \mathbf{q}_{2}\right) \omega_{1} \mathbf{k}, \omega_{1} \mathbf{k}\right\rangle \\
& \quad=\omega_{1} \omega_{2}\left\langle\left\langle\left(\mathbf{k} \times \mathbf{q}_{1}, \mathbf{k} \times \mathbf{q}_{2}\right),\left(\mathbf{k} \times \mathbf{q}_{1}, \mathbf{k} \times \mathbf{q}_{2}\right)\right\rangle\right\rangle
\end{aligned}
$$




$$
=\omega_{1} \omega_{2}\left\{m_{1}\left\|\mathbf{k} \times \mathbf{q}_{1}\right\|^{2}+m_{2}\left\|\mathbf{k} \times\left(\mathbf{q}_{1}+\mathbf{q}_{2}\right)\right\|^{2}\right\},
$$

with the result $\mathrm{I}\left(\mathbf{q}_{1}, \mathbf{q}_{2}\right)=m_{1}\left\|\mathbf{q}_{1}^{\perp}\right\|^{2}+m_{2}\left\|\left(\mathbf{q}_{1}+\mathbf{q}_{2}\right)^{\perp}\right\|^{2}$ being the moment of inertia of the system about the $\mathbf{k}$ axis, where $\left\|\mathbf{q}_{1}^{\perp}\right\|^{2}=\left\|\mathbf{q}_{1}\right\|^{2}-\left\|\mathbf{q}_{1} \cdot \mathbf{k}\right\|^{2}$ is the square length of the projection of $\mathbf{q}_{1}$ onto the $x-y$ plane. The mechanical connection is calculated using Eq. (5.16) to produce

$$
\begin{aligned}
\mathcal{A}\left(\mathbf{q}_{1}, \mathbf{q}_{2}, \mathbf{v}_{1}, \mathbf{v}_{2}\right)=\mathbb{I}^{-1} \mathbf{J}= & \mathbb{I}^{-1}\left\{\mathbf { k } \cdot \left[m_{1} \mathbf{q}_{1} \times \mathbf{v}_{1}\right.\right. \\
& \left.\left.+m_{2}\left(\mathbf{q}_{1}+\mathbf{q}_{2}\right) \times\left(\mathbf{v}_{1}+\mathbf{v}_{2}\right)\right]\right\} .
\end{aligned}
$$

Therefore, the amended potential is

$$
\begin{aligned}
V_{\mu}\left(\mathbf{q}_{1}, \mathbf{q}_{2}\right)= & m_{1} g \mathbf{q}_{1} \cdot \mathbf{k}+m_{2} g\left(\mathbf{q}_{1}+\mathbf{q}_{2}\right) \cdot \mathbf{k} \\
& +\frac{\mu^{2} / 2}{m_{1}\left\|\mathbf{q}_{1}^{\perp}\right\|^{2}+m_{2}\left\|\left(\mathbf{q}_{1}+\mathbf{q}_{2}\right)^{\perp}\right\|^{2}} .
\end{aligned}
$$

The relative equilibria are computed by finding the critical points of $V_{\mu}$. The obvious relative equilibria with $\mathbf{q}_{1}^{\perp}=\mathbf{0}$ and $\mathbf{q}_{2}^{\perp}=\mathbf{0}$, in which individual pendula point vertically upwards or downwards, are singular and not of interest here. Rather, we search for solutions pointing downwards with $\mathbf{q}_{1}^{\perp} \neq \mathbf{0}$ and $\mathbf{q}_{2}^{\perp} \neq \mathbf{0}$. Since in $\left(\mathbf{q}_{1}^{\perp}, \mathbf{q}_{2}^{\perp}\right)$ coordinates

$$
\begin{aligned}
V_{\mu}\left(\mathbf{q}_{1}^{\perp}, \mathbf{q}_{2}^{\perp}\right)= & -\left(m_{1}+m_{2}\right) g \sqrt{l_{1}^{2}-\left\|\mathbf{q}_{1}^{\perp}\right\|^{2}} \\
& -m_{2} g \sqrt{l_{2}^{2}-\left\|\mathbf{q}_{2}^{\perp}\right\|^{2}}+\frac{1}{2} \frac{\mu^{2}}{I},
\end{aligned}
$$

its extrema yield relative equilibria given by the points of the graph of

$$
\lambda^{2}=\frac{L^{2}-r^{2}}{L^{2}-\alpha^{2}}, \quad \text { where } L=\left(1+\frac{\alpha}{m}\right)\left(\frac{\alpha}{1+\alpha}\right),
$$

with the restriction $0 \leqslant \lambda^{2} \leqslant r^{2} / \alpha^{2}$ and definitions $\alpha$ $=\mathbf{q}_{2}^{\perp} / \mathbf{q}_{1}^{\perp}, \lambda=\left\|\mathbf{q}_{1}^{\perp}\right\| / l_{1}, r=l_{2} / l_{1}, m=\left(m_{1}+m_{2}\right) / m_{2}$.

It is easier to analyze the solution by rewriting $\mathbf{q}_{1}^{\perp}, \mathbf{q}_{2}^{\perp}$ in polar coordinates $\left(r_{1}, \theta_{1}\right)$ and $\left(r_{2}, \theta_{2}\right)$ with $\phi=\theta_{2}-\theta_{1}$ being an $S^{1}$-invariant coordinate. The functions $\phi, r_{1}, r_{2}$ form a coordinate chart on the shape space, i.e., they can be regarded as $G$-invariant functions on the configuration space. At relative equilibria, both pendula have to lie in the same vertical plane through the origin. Therefore, the value of the coordinate $\phi$ is either 0 or $\pi$. Thus, the internal configuration of the system is determined by the parameter $\alpha$ : if $\alpha>0$, we get a straight-stretched-out solution with $\phi=0$, whereas if $\alpha<0$, the solution is of the "cowboy" type with $\phi=\pi$.

\section{Energy-momentum method}

As a result of the above cotangent bundle reduction, we arrive at the augmented Hamiltonian

$$
H_{\xi}(\mathbf{z})=H(\mathbf{z})-\langle\mathbf{J}(\mathbf{z})-\mu, \xi\rangle=K_{\mu}(\mathbf{z})+V_{\mu}(q),
$$

where $V_{\mu}$ is the amended potential, $K_{\mu}(\mathbf{z})=\frac{1}{2} \| p$ $-\alpha_{\mu}(q) \|^{2}$ is the amended kinetic energy, and $\mu \in \mathfrak{g}^{*}$ and $\xi \in \mathfrak{g}$ are related by the locked inertia tensor $\mathbb{I}(q): \mathfrak{g}_{\mu} \mapsto \mathfrak{g}_{\mu}^{*}$, as discussed in Sec. V.B.
Consider a relative equilibrium $\mathbf{z}_{e} \in P, \mu=\mathbf{J}\left(\mathbf{z}_{e}\right)$; thus, there exists $\xi \in \mathfrak{g}$ such that $\mathbf{z}_{e}$ is a critical point of the augmented Hamiltonian $H_{\xi}(\mathbf{z})=H(\mathbf{z})-\langle\mathbf{J}(\mathbf{z})-\mu, \xi\rangle$, i.e., $\delta H_{\xi}\left(\mathbf{z}_{e}\right)=0$. This is same as $\mathbf{z}_{e}$ being a critical point of the energy-momentum map $H \times \mathbf{J}: P \rightarrow \mathbb{R} \times \mathfrak{g}^{*}$. Next, we introduce a subspace $\mathcal{S} \subset T_{\mathbf{z}_{e}} P$, which is also $\mathcal{S} \subset \operatorname{ker} \mathbf{D J}\left(\mathbf{z}_{e}\right)$ and is transverse to the $G_{\mu}$ orbit within $\operatorname{ker} \mathbf{D J}\left(\mathbf{z}_{e}\right)$.

Theorem 7 (Energy-momentum theorem) (Marsden et al., 1989; Simo et al., 1991; Marsden, 1992). If $\delta^{2} H_{\xi}\left(\mathbf{z}_{e}\right)$ is definite on $\mathcal{S}$, then $\mathbf{z}_{e}$ is $G_{\mu}$ orbitally stable in $\mathbf{J}^{-1}(\mu)$ and $G$ orbitally stable in $P$.

The space of admissible configuration variations modulo variations generated by $\mathfrak{g}_{\mu}$ is denoted by $\mathcal{V}$. That is, if $\mathcal{N}$ consists of the vectors tangent to the $G_{\mu}$ orbit of $q_{e}$, then $\mathcal{V}$ is a complement of $\mathcal{N}$ in $T_{q_{e}} \mathrm{Q}$. The key idea is to split $\mathcal{V}=\mathcal{V}_{\text {rig }} \oplus \mathcal{V}_{\text {int }}$, i.e., rigid (group or rotational) and internal (vibrational) variations. If $\mathfrak{g}_{\mu}^{\perp}$ is the orthogonal complement to $\mathfrak{g}_{\mu}$ in $\mathfrak{g}$ with respect to the locked inertia metric, then $\mathcal{N}_{\text {rig }}=\left\{\eta_{\mathrm{Q}}(q) \in T_{q} \mathrm{Q} \mid \eta \in \mathfrak{g}_{\mu}^{\perp}\right\}$. The split $\mathcal{V}$ $=\mathcal{V}_{\text {rig }} \oplus \mathcal{V}_{\text {int }}$ induces a split of the phase space $S=S_{\text {rig }}$ $\oplus S_{\text {int }}$. Then, if the energy-momentum method is applied to simple mechanical systems with separable Hamiltonian $H=K+V$, there are coordinates in which $\delta^{2} H_{\xi}$ block diagonalizes (Marsden et al., 1989; Simo et al., 1991; Marsden, 1992),

$\delta^{2} H_{\xi}=\left[\begin{array}{cc}{\left[\begin{array}{c}\text { Rigid body } \\ \text { block }\end{array}\right]} & 0 \\ 0 & {\left[\begin{array}{c}\text { Internal vibrations } \\ \text { block }\end{array}\right]}\end{array}\right]$,

where the rigid body block corresponds to the Arnold (1971) form $A_{\mu}=\delta^{2} V_{\mu} \mid \mathcal{\nu}_{\text {rig }} \times \mathcal{V}_{\text {rig }}$, for the special case Q $=G$, and the internal vibrations block is simply $\operatorname{diag}\left[\delta^{2} V_{\mu} \mid \mathcal{v}_{\text {int }} \times \nu_{\text {int }}, \delta^{2} K_{\mu}\right]$.

If the symmetry group is Abelian, then $\mathfrak{g}_{\mu}=\mathfrak{g}$ and $\mathfrak{g}_{\mu}^{\perp}$ $=\{0\}$, so that $\mathcal{V}_{\text {rig }}=\mathfrak{g}_{\mu}^{\perp} \cdot q_{e}=\{0\}$. Therefore, the Arnold form vanishes $A_{\mu}=0$ and the resulting linearized equations of motion correspond to Eq. (2.4), so that the influence of dissipation can be easily accounted by the Thomson-Tait-Chetayev theory. On the other hand, if the symmetry group is non-Abelian, then $A_{\mu} \neq 0$. The main result of the work by Bloch et al. (1994) applies to the general case of non-Abelian symmetries: if $\delta^{2} H_{\xi}$ is indefinite, then the relative equilibrium gets destabilized after the addition of dissipation. This is the main message of this subsection, which is apparently built on a fair amount of geometry, some of which is introduced above. In classical times, when the results by ThomsonTait-Chetayev and Merkin were developed, these geometric underpinnings were not available. One of the examples of a non-Abelian group is the rotation group $\mathrm{SO}(3)$, that is, the order in which rotations are composed makes a difference. For example, a quarter turn around the positive $x$ axis followed by a quarter turn around the positive $y$ axis is a different rotation from the one obtained by first rotating around $y$ and then $x$. For illustration of the use of the energy-momentum method in de- 
termining the amended potential, we consider an example with the Abelian group.

Example (double spherical pendulum, Fig. 19). The stability analysis amounts to the computation of $\delta^{2} V_{\mu}$ on the subspace orthogonal to the $G_{\mu}$ orbit. It is easier to perform this task by rewriting $\mathbf{q}_{1}^{\perp}, \mathbf{q}_{2}^{\perp}$ in polar coordinates $\left(r_{1}, \theta_{1}\right)$ and $\left(r_{2}, \theta_{2}\right)$ with $\phi=\theta_{2}-\theta_{1}$ being an $S^{1}$-invariant coordinate. Then, the amended potential is given by

$$
\begin{aligned}
V_{\mu}= & -m_{1} g \sqrt{l_{1}^{2}-r_{1}^{2}}-m_{2} g\left(\sqrt{l_{1}^{2}-r_{1}^{2}}+\sqrt{l_{2}^{2}-r_{2}^{2}}\right) \\
& +\frac{1}{2} \frac{\mu^{2}}{m_{1} r_{1}^{2}+m_{2}\left(r_{1}^{2}+r_{2}^{2}+2 r_{1} r_{2} \cos \phi\right)},
\end{aligned}
$$

so that

$$
\delta^{2} V_{\mu}=\left[\begin{array}{lll}
a & b & 0 \\
b & d & 0 \\
0 & 0 & e
\end{array}\right],
$$

where

$$
\begin{aligned}
& a=\frac{\mu^{2}\left[3(m+\alpha)^{2}-\alpha^{2}(m-1)\right]}{\lambda^{4} l_{1}^{4} m_{2}\left(m+\alpha^{2}+2 \alpha\right)^{3}}+\frac{g m_{2} m}{l_{1}\left(1-\lambda^{2}\right)^{3 / 2}}, \\
& b=(\operatorname{sgn} \alpha) \frac{\mu^{2}}{\lambda^{4} l_{1}^{4} m_{2}} \frac{3\left(m+\alpha^{2}+2 \alpha\right)+4 \alpha(m-1)}{\left(m+\alpha^{2}+2 \alpha\right)^{3}}, \\
& d=\frac{\mu^{2}}{\lambda^{4} l_{1}^{4} m_{2}} \frac{3(\alpha+1)^{2}+1-m}{\left(m+\alpha^{2}+2 \alpha\right)^{3}}+\frac{m_{2} g}{l_{1}} \frac{r^{2}}{\left(r^{2}-\lambda^{2} \alpha^{2}\right)^{3 / 2}}, \\
& e=\frac{\mu^{2}}{\lambda^{2} l_{1}^{2} m_{2}} \frac{\alpha}{\left(m+\alpha^{2}+2 \alpha\right)^{3}} .
\end{aligned}
$$

From here it follows that the straight-stretched-out branch of the double spherical pendulum (with $\alpha>0$ ) is stable, while the cowboy branch has an indefinite second variation, and thus is susceptible to dissipation-induced instabilities.

\section{Summary}

One can conclude that in certain situations, when relative equilibria can be reduced to the ordinary one, the stability of relative equilibria can be studied with the help of the classical Thomson-Tait-Chetayev theory. Since the existence of relative equilibria presumes the presence of symmetries and thus conserved quantities, stability can be naturally proved with the use of the energy-momentum method. If, however, the symplectic structure is singular, then the existence of the corresponding constants of motion-Casimirs, i.e., functions $C$ that Poisson commute with every function $F:\{C, F\}$ $=0$-allows the stability to be demonstrated showing the strict convexity using the energy-Casimir method. However, in this paper we are primarily interested in the instability phenomena, which are usually established on the basis of Lyapunov's linearization theorem. The proof of stability is necessary, however, in order to establish the existence of the bifurcation point rigorously (recall

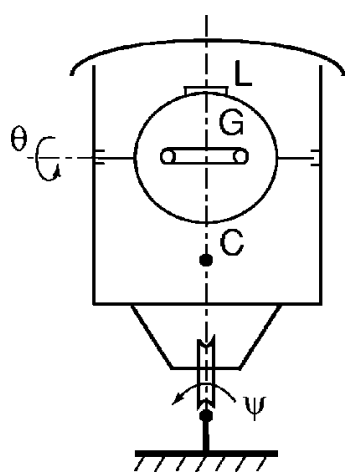

FIG. 20. Monorail car.

that the location of all eigenvalues on the imaginary axis is a necessary condition for stability of Hamiltonian systems, but is not sufficient, and thus requires more intricate analysis with the help of these methods). What is the most relevant to our discussion here is the fact that the Thomson-Tait-Chetayev and Merkin theorems allow one to establish dissipation-induced instability if the Hamiltonian system has indefinite and definite second variation, respectively, and is of the form (2.4). Evidently, the form of the system in Eq. (2.4) implies that the equilibrium is ordinary, but the same form (2.4) applies to certain cases of relative equilibria after the change of variables (reduction). A more general situation of relative equilibria originating from non-Abelian symmetries is accounted by the theory of Bloch et al. (1994), which is a natural extension of the classical Thomson-Tait-Chetayev theory.

\section{CONTROLLING DISSIPATION-INDUCED INSTABILITIES}

In this section, the question of controlling dissipationinduced instabilities both from classical and modern geometric viewpoints is discussed.

\section{A. Classical approach}

At the most trivial level, the structure of the linear part of system (2.4) suggests simply to add the appropriate linear forces that stabilize the equilibria; see, e.g., Borisenko et al. (2001). In many engineering applications, this is a widespread approach, as illustrated with the example of the monorail car, schematically shown in Fig. 20.

As we know from the necessary condition for gyroscopic stabilization, the number of degrees of instabilities should be even, while the monorail car apparently has an odd degree-the angle $\psi$ defining the deviation of the car from the vertical plane. To achieve gyroscopic stabilization, one needs to make the second coordinate-the angle of rotation of the gyroscope ring $\theta$-unstable by placing a load $L$ to the top of the ring as depicted in Fig. 20. However, as follows from the Thomson-Tait-Chetayev theorem, the achieved stability is temporary since the dissipation coming from friction 
makes the vertical position of the car unstable. The engineering solution to this problem is to create an angular momentum that would act in the direction of rotation of the ring and be proportional to the angular velocity $\dot{\theta}$. This is one of the oldest examples that was resolved with the help of the classical theory. Below we discuss more modern approaches to controlling dissipation-induced instabilities.

\section{B. Geometric control}

The basic idea of this discussion follows from a simple observation: dissipation-induced instabilities of the Thomson-Tait-Chetayev type happen only if $\delta^{2} H\left(z_{e}\right)$ is indefinite. Therefore, to avoid this instability, we can modify the original Hamiltonian system so that $\delta^{2} H\left(z_{e}\right)$ becomes definite. Therefore, more involved control techniques compared to the above compensators are based on reshaping either potential (Jalnapurkar and Marsden, 2000) or kinetic (Bloch et al., 1997) energies through feedback. While the former is done in the Hamiltonian context, the latter is devoted to the Lagrangian approach. Here we discuss the elements of the geometric control in the Hamiltonian setting, since it exploits the ideas of the previous sections. In general, it should be kept in mind that the control in the Lagrangian setting is equivalent to its Hamiltonian counterpart under rather general hypotheses, as shown by Chang et al. (2002).

\section{General methodology}

In the context of the general finite-dimensional nonlinear control system $\dot{x}=f(x, u)$ on a smooth $n$-manifold $M$, an affine Hamiltonian control system on a Poisson manifold $M=P$ has the form

$$
\dot{x}=X_{H_{0}}(x)+\sum_{j=1}^{m} X_{F_{j}}(x) u_{j}, \quad x \in M,
$$

where $H_{0}, F_{1}, \ldots, F_{m}$ are smooth functions on $P, X_{H_{0}}$ is the (Hamiltonian) drift vector field and $X_{F_{i}}, j=1, \ldots, m$, are (Hamiltonian) control vector fields corresponding to $F_{j}$, and the admissible map to the constrained set $\Omega$, $u(t): \mathbb{R}^{+} \rightarrow \Omega \subset \mathbb{R}^{m}$ is piecewise smooth. The system (6.1) is said to be underactuated if $m<n$. The most important issue of the control problem (6.1) is controllability, i.e., whether one can drive the system from one point to another with the given class of admissible controls $u(t)$. The controllability is closely related and in many cases can be proved through the system accessibility. The accessibility distribution ${ }^{17}$ is the distribution generated by vector fields in the accessibility algebra $\mathcal{C}$, which is a linear space and is just the span of all possible brackets of $X_{H_{0}}$ and $X_{F_{j}}$. If the dimension of the codistribution

\footnotetext{
${ }^{17} \mathrm{~A}$ smooth distribution on a manifold $M$ is the assignment to each point $x \in M$ of a subspace spanned by a set of smooth vector fields at $x \in M$.
}

$\mathrm{d} \mathcal{C}(z)=\operatorname{span}\{\mathrm{d} g(z) \mid g \in \mathcal{C}\}$ is $\operatorname{dim} \mathrm{d} \mathcal{C}=2 n$, then the system (6.1) is strongly accessible.

Further, we assume that at the equilibrium $z_{0}$, defined by $\mathrm{d} H_{0}\left(z_{0}\right)=0$, we have $F_{i}\left(z_{0}\right)=0, j=1, \ldots, m$. Since the second derivative $\delta^{2} H\left(z_{0}\right)$ at the isolated equilibrium is intrinsically defined, there are two main possibilities to consider. In the case in which $\delta^{2} H\left(z_{0}\right)$ is positive definite, $z_{0}$ is a strict minimum of $H$. As proven by Nijmeijer and van der Schaft (1996), if the codistribution $\mathrm{d} \mathcal{C}$ is of dimension $\operatorname{dim} P$ on a neighborhood of $z_{0}$, then the feedback $u_{i}=k_{i} X_{F_{i}}[H], k_{i}>0$, makes $z_{0}$ an asymptotically stable equilibrium. Moreover, if the functions $F_{i}$ commute, i.e., $\left\{F_{i}, F_{j}\right\}=0$, the feedback can be expressed in the form $u_{i}=-k_{i} \dot{F}_{i}$, which is easy to show from Eq. (6.1).

The more interesting situation corresponds to $\delta^{2} H\left(z_{0}\right)$ indefinite. Introducing a new feedback of the form $u_{i}(z)=-c_{i} F_{i}(z)+v_{i}$, where constants $c_{i}>0$, and noting that $c_{i} F_{i}(z) X_{F_{i}}(z)=c_{i} X_{(1 / 2) F_{i}^{2}}(z)$, we rewrite the system (6.1) as

$$
\dot{x}=X_{\tilde{H}}(x)+\sum_{j=1}^{m} X_{F_{j}}(x) v_{j}, \quad x \in M,
$$

where

$$
\tilde{H}=H+\sum_{i} c_{i}(1 / 2) F_{i}^{2}
$$

is the modified Hamiltonian. Since $F_{i}\left(z_{0}\right)=0$, the point $z_{0}$ is a critical one for $\tilde{H}(z)$. As proven by van der Shaft (1986), if $\delta^{2} H\left(z_{0}\right)$ is positive definite on $\operatorname{ker} \mathrm{d} F\left(z_{0}\right)$, then one can find positive constants $c_{i}$, such that $\delta^{2} \tilde{H}\left(z_{0}\right)$ is positive definite. Intuitively, this implies that we need actuation along all directions on which the second variation is not positive definite. This enables us to use the same theorem of Nijmeijer and van der Schaft (1996), which was discussed above for the case $\delta^{2} H\left(z_{0}\right)>0$, but now is used with a new set of functions $\tilde{\mathcal{C}}=\mathcal{C}(H \rightarrow \tilde{H})$. As a result, the feedback of the proportional derivative form $u_{i}=-c_{i} F_{i}-k_{i} \dot{F}_{i}$ makes $z_{0}$ an asymptotically stable equilibrium. The proportional term $-c_{i} F_{i}$ modifies the potential and converts the equilibrium to a minimum of the modified Hamiltonian, while the derivative term $-k_{i} \dot{F}_{i}$ is used to introduce dissipation in the system and thereby achieve an asymptotic stability.

\section{Application to systems with symmetry}

Following Jalnapurkar and Marsden (2002), we apply this theory to mechanical systems with symmetry and illustrate using the double spherical pendulum. Keeping this example in mind, we discard the Arnold form $A_{\mu}$ $=\left.\delta^{2} V_{\mu}\left(q_{e}\right)\right|_{\mathcal{V}_{\text {rig }}}$ in Eq. (5.28) in view of Abelian symmetry. Therefore, we have $\mathcal{V}=\mathcal{V}_{\text {int }}$, so that we are left with 


$$
\delta^{2} H_{\mu}=\left[\begin{array}{cc}
B_{\mu} & 0 \\
0 & K_{\mu}
\end{array}\right],
$$

where $B_{\mu}=\left.\delta^{2} V_{\mu}\left(q_{e}\right)\right|_{\mathcal{V}_{\text {int }}}$ and $K_{\mu}$ is a matrix of size $\operatorname{dim} S \times \operatorname{dim} S$ that depends on the kinetic energy metric only and is known to be positive definite. We assume that $B_{\mu}$ is not positive definite, and thus we apply the procedure from Sec. VI.B.1 to form a modified Hamiltonian (6.3), which leads to the modification of the potential energy, $\tilde{V}_{\mu}=V_{\mu}+(1 / 2) \Sigma_{i} c_{i} F_{i}^{2}$. Since $\delta^{2}\left(\Sigma_{i} c_{i} F_{i}^{2}\right)$ $=\left[\mathrm{d} F\left(q_{e}\right)\right]^{T} C \mathrm{~d} F\left(q_{e}\right)$, with $C=\operatorname{diag}\left\{c_{1}, \ldots, c_{m}\right\}$, and denoting by $K$ the matrix of $\mathrm{d} F\left(q_{e}\right): \mathcal{V} \rightarrow \mathbb{R}^{m}$, the blockdiagonal form is transformed to

$$
\delta^{2} \tilde{H}_{\mu}=\left[\begin{array}{cc}
B_{\mu}+K^{T} C K & 0 \\
0 & K_{\mu}
\end{array}\right] .
$$

As proven by Jalnapurkar and Marsden (2002), $B_{\mu}$ $+K^{T} C K$ is positive definite iff $B_{\mu}$ is positive definite on ker $K$. The requirement of the van der Shaft theorem (Nijmeijer and van der Schaft, 1996) that the codistribution should be of maximal dimension, i.e., of the dimension of $\left(T^{*} \mathrm{Q}\right)_{\mu}$, has been verified by Jalnapurkar and Marsden (2002).

Example (double spherical pendulum, Fig. 19). Recalling that $\phi, r_{1}, r_{2}$ are coordinates on the shape space, after diagonalization the $3 \times 3$ matrix $B_{\mu}=\delta^{2} V_{\mu}\left(q_{e}\right)$ has only one positive entry. Since we need $B_{\mu}$ to be positive definite on $\operatorname{ker} \mathrm{d} F\left(q_{e}\right): \mathcal{V} \rightarrow \mathbb{R}^{m}, \operatorname{ker} \mathrm{d} F\left(q_{e}\right)$ can have dimension at most 1 , and thus $F$ needs to have at least two components, since we must apply actuation in all directions along which the second derivative of the Hamiltonian is not positive definite. Therefore, let $m=2$ and choose $F_{1}=r_{1}$ and $F_{2}=\phi$. Note that $F_{2}$ is not continuous everywhere on the shape space, but for studying local behavior in the neighborhood of relative equilibrium, this is not an issue. Our system is underactuated since the shape space has dimension equal to 3 , while the control input is of dimension $m=2$. The matrix $\mathrm{d} F\left(q_{e}\right): \mathcal{V}$ $\rightarrow \mathbb{R}^{2}$ is

$$
K=\left[\begin{array}{lll}
\frac{\partial F_{1}}{\partial r_{1}}\left(q_{e}\right) & \frac{\partial F_{1}}{\partial r_{2}}\left(q_{e}\right) & \frac{\partial F_{1}}{\partial \phi}\left(q_{e}\right) \\
\frac{\partial F_{2}}{\partial r_{1}}\left(q_{e}\right) & \frac{\partial F_{2}}{\partial r_{2}}\left(q_{e}\right) & \frac{\partial F_{2}}{\partial \phi}\left(q_{e}\right)
\end{array}\right]=\left[\begin{array}{lll}
1 & 0 & 0 \\
0 & 0 & 1
\end{array}\right],
$$

with ker $K=\operatorname{span}\left\{\left[\begin{array}{lll}0 & 1 & 0\end{array}\right]^{T}\right\}$. If $B_{\mu}$ is positive definite on ker $K$, it is possible to find $c_{1}, c_{2}$ such that $\left.\delta^{2} \tilde{V}_{\mu}\left(q_{e}\right)\right|_{\nu}$ is positive definite. Thus, we need to check if $\partial^{2} V_{\mu} / \partial r_{2}^{2}\left(q_{e}\right)$ is positive.

Let us assume that both rods are of unit length, and both bobs are of unit mass, and choose $\alpha=-3 / 2$, which corresponds to a cowboy solution. The amended potential $V_{\mu}$ is given by Eq. (5.24), and it is easy to verify that $\partial^{2} V_{\mu} / \partial r_{2}^{2}\left(q_{e}\right)$ is positive. This ensures that there exist constants $c_{1}$ and $c_{2}$ such that $\left.\delta^{2} \tilde{V}_{\mu}\left(q_{e}\right)\right|_{\mathcal{V}}$ is positive definite. For example, the choice $c_{1}=300$ and $c_{2}=20$ will work, and the corresponding feedback law $u_{1}=300 r_{1}$ $-k_{1} \dot{r}_{1}$ and $u_{2}=20 r_{2}-k_{2} \dot{\phi}$ will make the cowboy solution asymptotically stable relative equilibrium for any choice of positive constants.

\section{Remarks}

In this section, only the control relevant to the Thomson-Tait-Chetayev theory was discussed, while Merkin's case was not explored. Another interesting problem would be to develop control theory of the Hamiltonian Hopf 1:1 resonance, since the literature is concerned only with the dissipative case of 1:1 resonance, as in the works of Abed and collaborators (Abed and Fu, 1986; Liaw and Abed, 1990, 1996).

\section{TOWARDS AN INFINITE-DIMENSIONAL THEORY}

As indicated throughout, dissipation-induced instabilities are now well understood in the case of finitedimensional mathematical description. While this description models and reflects appropriately the behavior of underlying physical systems, one needs to keep in mind that the real physical systems are always infinite dimensional; for instance, the friction of the Lagrange top with either air or solid surface (hinge) is of an infinite-dimensiona ${ }^{18}$ nature, but the finite-dimensional description of it is just a good and successful approximation. In this sense we distinguish this class of physical systems as "finite dimensional," while the strict meaning is attached only to the type of mathematical description. In this context, the radiation-induced instability discussed in Sec. III.A.1 might be thought of as an infinitedimensional example (3.16) (coupling of a finite degree of freedom mechanical system to an infinite-dimensional wave equation), but as analysis revealed the underlying dynamics is finite-dimensional (3.17). In this concluding section, we address the question of the presence of dissipation-induced instabilities in truly infinitedimensional systems, i.e., which cannot be easily approximated with finite-dimensional models. First, we provide a motivating physical example, and next we outline the general points pertinent to all infinitedimensional systems.

\section{A. Baroclinic instability}

One example of this type-a baroclinic instability in atmospheric and ocean dynamics-was recently developed by Krechetnikov and Marsden (2005). The baroclinic instability is a large-scale instability of the westerly winds in midlatitudes, when the basic (equilibrium) state has a vertical shear $\psi_{i}^{e}=-U_{i}^{e} y$ as shown in Fig. 21. In this mathematical idealization, the origin of the basic state is unspecified and the model reflects the fact that this basic state is maintained against dissipative effects by an external source of energy. Physically, this particular basic state results from a temperature gradient between the

\footnotetext{
${ }^{18}$ Instead of infinite-dimensional we use adjectives extended or continuous, as is common in the literature.
} 


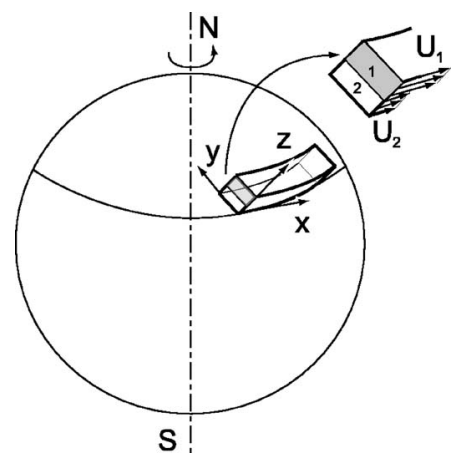

FIG. 21. Physical domain and basic state $U_{i}$ on the surface of a rotating planet.

subtropical and polar regions, which causes a pressure gradient aloft. The latter is balanced by the Coriolis force to form a geostrophic flow known as the Westerlies. The instability of these baroclinic zonal currents has been a subject of numerous studies and is known to occur as a result of a release of available potential energy of sloping density surfaces.

The paradigm used in our study is the quasigeostrophic two-layer $\beta$-plane model introduced by Phillips (1951), namely,

$$
\partial_{t} q_{i}+\mathbf{v}_{i} \cdot \nabla q_{i}=-r \nabla^{2} \psi_{i}, \quad(x, y) \in \mathbf{D}, \quad i=1,2,
$$

with no summation over $i$. This model, whose notation is explained below, accounts for the large-scale evolution in midlatitudes with the simplified effects of the Earth's rotation and sphericity ( $\beta$ effect), stratification (modeled by two-layer approximation) with internal rotational Froude number $F$, and Eckman layer dissipation $(r \geqslant 0)$. Equation (7.1) is posed on a rectangular domain, D $=\{-1 \leqslant x \leqslant 1 ; 0 \leqslant y \leqslant 1\}$, located on the surface of a rotat ing planet as shown in Fig. 21. In the formulation (7.1), we have used the usual definition of potential vorticity, namely, $q_{i}=\nabla^{2} \psi_{i}+(-1)^{i} F\left(\psi_{1}-\psi_{2}\right)+\beta y$, where the stream functions $\psi_{i}$ in the $i$ th layer are related to the velocities by $\mathbf{v}_{i}=(u, v)_{i}=\mathbf{e}_{z} \times \boldsymbol{\nabla} \psi_{i}=\left(-\partial_{y} \psi_{i}, \partial_{x} \psi_{i}\right)$, and where the twodimensional gradient is $\boldsymbol{\nabla}=\mathbf{i} \partial_{x}+\mathbf{j} \partial_{y}$. The left-hand side of Eq. (7.1) is the usual material (Euler) transport of potential vorticity, while the right-hand side is the Eckman layer dissipation, where $r \geqslant 0$. The problem (7.1) is treated here with the boundary conditions corresponding to a Phillips model, i.e., periodicity in $x$ and nopenetration condition at $y=0,1$. For further details, we refer the reader to the work of Pedlosky (1987).

The unexpected destabilizing effect due to the introduction of friction was vindicated, in particular, in the linear stability study of Romea (1977), who demonstrated that an introduction of dissipation leads to an $O(1)$ destabilization effect. However, no attempt to prove the presence of a dissipation-induced instability in a sense of Definition 2 has been made. As our previous study (Krechetnikov and Marsden, 2005) demonstrated, the dissipation-induced instability develops according to scenario (3.29b) in analogy with positional forces in the finite-dimensional case. This constitutes the main result of Krechetnikov and Marsden (2005):

Theorem 8. The equilibrium $\psi_{i}^{e}=-U_{i}^{e} y$ of the Hamiltonian quasigeostrophic two-layer $\beta$-plane system (7.1) with $r=0$ experiences a dissipation-induced instability in the parameter range $[2(1+\sqrt{2})]^{-1 / 2}<\left|U_{1}-U_{2}\right| F / 2 \beta<1 / 2$ in a sense of Definition 2 when an arbitrarily small dissipation effect, $r>0$, is added. Moreover, this equilibrium of the Hamiltonian system (that is, with $r=0$ ) is Lyapunov stable in the above parameter range.

From the physical viewpoint, this result implies that if one is predicting the appearance of a baroclinic instability by measuring the velocity difference $U_{c}=\left|U_{1}-U_{2}\right|$ based on the Hamiltonian formulation, the error of predicting the critical bifurcation parameter will be around $10 \%$. Though this difference is probably within the accuracy of meteorological forecasts, it is still of physical and mathematical importance: we believe that this phenomenon is more frequent than rare and its prominence may vary depending upon a particular problem at hand.

\section{B. General issues}

From a physical standpoint, one can anticipate that dissipation-induced instability phenomena should take place in other truly infinite-dimensional systems. However, the lack of classification of forces and of their identification in the mathematical formalism, analogous to the finite-dimensional case, does not allow one to systematize the various types of dissipation-induced instabilities based on force classification (dissipative versus positional), as done for finite dimensions in Sec. III. Indeed, an infinite-dimensional description usually comes after some kind of coarsening procedure has been applied to a system with an infinite number of degrees of freedom (e.g., averaging over fluid particles when deducing Navier-Stokes equations) and often after a certain symmetry reduction (e.g., removing a particle-relabeling symmetry when deriving Euler equations for ideal fluid). The latter obscures the physical interpretation of various terms in the resulting equations compared to finitedimensional mechanical systems (2.4). However, the geometric picture introduced in Sec. III suggests to build the classification upon the definiteness of the second variation $\delta^{2} H$ of the disturbance dynamics, as depicted in Fig. 5. Similar to the finite-dimensional case, the two situations correspond to definite and indefinite $\delta^{2} H$.

From a mathematical standpoint one can anticipate a number of complications. First of all, the addition of dissipation usually introduces higher-order derivatives in the partial differential equation models, as in the case of the addition of viscosity to the Euler equations, which results in the Navier-Stokes equations. The presence of such higher-order derivatives can lead to a modification of a given equilibrium solution (i.e., basic state in fluid mechanics terminology) and thus complicates the interpretation of a stability analysis. However, the destabilizing effect of viscosity, which is obviously responsible for dissipation, was noticed a long time ago, cf. Lin (1955), 
but explaining this effect is not a trivial problem: even transition to turbulence in a simple geometry such as a channel or pipe (Poiseuille flow) is a challenge that is still not fully resolved. Last, even when the equilibrium solution remains unmodified after dissipation is added, both components of the analysis-(in)stability and existence of solutions - and their interrelation provide other sources of intricacy, as discussed below.

As we know, in proving instability one relies heavily upon Lyapunov's indirect (linear approximation) method, which is well justified in the finite-dimensional case, but in general is not valid in infinite dimensions (Luo et al., 1999). This difficulty might be overcome with the help of Daleckii and Krein (1974) and Yudovich (1989) theories (Yudovich's version is more advanced than Krein's), which allow one to establish the connection between linear and nonlinear (in)stability under specific conditions on linear and nonlinear operators. While the general theory is quite involved, to get a feeling of limitations of the theory compared to the finitedimensional case, consider a general nonlinear equation with a stationary principal part in Banach space,

$$
\mathrm{d} x / \mathrm{d} t=A x+F(x, t) .
$$

Then, if the spectrum $\sigma(A)$ does not intersect the imaginary axis but there are eigenvalues in the right halfplane, and there exists a number $q_{0}$ depending only on the operator $A$ such that the nonlinear operator satisfies $\|F(x, t)\| \leqslant q\|x\|$ for $q \leqslant q_{0}$ and $t \geqslant 0,\|x\| \leqslant \rho$, then the zero solution of the differential equation (7.2) is unstable for $t \rightarrow+\infty$. Here $\|\cdot\|$ stands for the norm in the Banach space.

Even after succeeding in establishing (in)stability, one meets with another complication-norm dependence of stability criteria-an issue that has been understood for a long time (Yudovich, 1989; Friedlander and Yudovich, 1999). In the finite-dimensional case, this difficulty cannot arise since all norms are equivalent. One of the simplest examples of this subtlety has been given by Yudovich (1989) and represents a linear partial differential equation,

$$
\begin{aligned}
& \frac{\partial u}{\partial t}=x \frac{\partial u}{\partial x}, \\
& u(0, x)=\phi(x),
\end{aligned}
$$

the unique solution of which is simply $u(x, t)=\phi\left(x e^{t}\right)$. Since $\left\|\partial^{k} u(\cdot, t) / \partial x^{k}\right\|_{L^{p}(\mathrm{R})}=e^{\left(k-p^{-1}\right) t}\left\|\phi^{(k)}\right\|_{L^{p}(\mathrm{R})}$, one has (i) asymptotic stability in $L^{p}(\mathrm{R})$ for $1 \leqslant p<\infty$, (ii) Lyapunov stability in $L^{\infty}(\mathbb{R})$, and (iii) exponential instability in Sobolev spaces $W^{k, p}(\mathbb{R})$ with $k>1, p \geqslant 1$ or $k=1, p>1$.

This brings up the last major issue one has to worry about, namely, choosing a physically relevant function space and proving the existence of the solution in the same function space in which stability is investigated. This task immediately uncovers the disjointedness of current methods for proving nonlinear stability [mainly Arnold's method, i.e., convexity estimates (Arnold, 1965, 1969; Holm et al., 1985)], and methods for proving the existence of solutions, which are usually based on a priori estimates. While the work of Krechetnikov and Marsden (2005) succeeded in establishing stability and existence in the same function space, the lack of elegance and efficiency becomes evident and suggests the need to develop new methods that would achieve a simultaneous study of both questions. In the next subsection, the major difficulties of studying existence and stability in a unified approach in the context of finitedimensional mechanical problems are highlighted: those difficulties become even more dramatic in the context of infinite-dimensional systems.

\section{On proving existence and stability}

Historically, the methods for proving existence and stability have been developing independently, and therefore it is natural that the techniques used often do not have much in common. In general, it is clear that the equilibrium solution may exist, but be unstable, which explains why existence methods are not tied up with stability methods. Therefore, the conditions obtained in the stability proof are generally of no use in the existence proof. This is the fundamental reason for these two methods to be disjoint. However, one might expect that the estimates found in the stability proof might facilitate the existence proof substantially and thus lead to a united method for proving both properties. We illustrate here that in some situations the assumptions necessary for proving stability and existence are basically the same, and therefore should help one in developing a united method to prove these two properties simultaneously in the same function space. While the way of achieving that has not been explored yet, it should be important for partial differential equations, where the function space setup becomes intricate for both stability and existence analyses.

The observation that proving nonlinear stability is of greatest importance for conservative systems, for which linear and spectral stabilities do not imply a nonlinear one, suggests that we should look first at this class of problems. ${ }^{19}$ Just for illustration, consider the following simple example, in which linear stability does not imply nonlinear one:

$$
\ddot{q}+q^{3}=0 .
$$

It is easy to see, by multiplying by $\dot{q}$ and integrating in time $t$, that this system is conservative with the energy of the form kinetic $T$ plus potential $V$ energy,

$$
E=\frac{\dot{q}^{2}}{2}+\frac{q^{4}}{4}=T+V(q) .
$$

\footnotetext{
${ }^{19}$ In the dissipative case, there are results by Daleckii and Krein (1974) and Yudovich (1989), which allow one to establish nonlinear (in)stability based on linearization.
} 


\section{Stability}

Let generalized momentum be $p=\dot{q}$, so that the Hamiltonian is $H=p^{2} / 2+V(q)$ with the resulting Hamiltonian equations

$$
\begin{aligned}
& \dot{p}=-\frac{\partial H}{\partial q}=-q^{3}, \\
& \dot{q}=\frac{\partial H}{\partial p}=p,
\end{aligned}
$$

or $\int \dot{\mathbf{z}}(t)+\nabla H(\mathbf{z}(t))=0$ with $\mathbf{z}=(q, p)$ and $H \in C^{1}\left(\mathbb{R}^{2}, \mathbb{R}\right)$. The equilibrium point is simply the origin of the phase space $\mathbf{z}=(q, p)=\mathbf{0}$ with $H(\mathbf{0})=0$, so that the linearized dynamics is given by

$$
\begin{aligned}
& \dot{p}=0, \\
& \dot{q}=p .
\end{aligned}
$$

Clearly, the system is spectrally stable with eigenvalues $\lambda_{1,2}=0$, but linearly unstable since for initial conditions $t=t_{0}:(q, p)=\left(q_{0}, p_{0}\right)$, the solution grows linearly in time,

$$
\begin{aligned}
& p=p_{0}, \\
& q=q_{0}+p_{0}\left(t-t_{0}\right) .
\end{aligned}
$$

Following Lyapunov's definition of stability, let initial conditions be chosen as in Eq. (2.10). Then the maximum possible energy is $H_{\max }=\delta^{2} / 2+\delta^{4} / 4$, while the energy of the evolving system is

$$
H=\frac{p(t)^{2}}{2}+\frac{q(t)^{4}}{4} \leqslant H_{\max }=\frac{\delta^{2}}{2}+\frac{\delta^{4}}{4} .
$$

Therefore, in view of convexity of $(q, p) \mapsto H(q, p)$,

$$
|p(t)| \leqslant\left(2 H_{\max }\right)^{1 / 2}, \quad|q(t)| \leqslant\left(4 H_{\max }\right)^{1 / 4},
$$

and $\epsilon$ in Eq. (2.11) is given by $\epsilon$ $=\max \left[\left(2 H_{\max }\right)^{1 / 2},\left(4 H_{\max }\right)^{1 / 4}\right]$. Thus, we have found explicit estimates for all the constants in the definition of Lyapunov stability and proved that the system is nonlinearly stable. We effectively used the Lyapunov direct method: the energy function is a Lyapunov function in this case, because it is positive definite and its time derivative vanishes. The stability, of course, can also be seen from the fact that the Hamiltonian is separable and the potential energy has a strict minimum at $q=0$, which is a global minimum here. It should be noted that the energy $E$ cannot be chosen as a norm since the property of homogeneity is not satisfied, i.e., $\|\lambda q\| \neq|\lambda|\|q\|$. However, in view of finite dimensionality of the problem, the stability result would hold in any norm. It should be stressed that the above proof is based on finding the appropriate energy estimate and convexity estimates, which bound the dynamics of each variable, $q(t)$ and $p(t)$. Since the proof involves only $q(t)$ and $\dot{q}(t) \equiv p$, then the appropriate function space is $q(t) \in C^{1}$.

\section{Existence}

The history of proving the existence of solutions for Hamiltonian systems is very rich. Starting with
Poincaré's initiative to treat this question with variational calculus, it has been tempting to move this problem into the Lagrangian realm, and to prove the existence of a solution by demonstrating the existence of a minimizer for a corresponding action. The associated Lagrangian for Eq. (7.3) is

$$
L(q, \dot{q})=\frac{\dot{q}^{2}}{2}-V(q),
$$

and the variational principle of Hamilton,

$$
\delta \int_{t_{0}}^{t_{1}} L(t, q, \dot{q}) \mathrm{d} t=0,
$$

yields the original Euler-Lagrange equation (7.3),

$$
\frac{\mathrm{d}}{\mathrm{d} t} \frac{\partial L}{\partial \dot{q}}-\frac{\partial L}{\partial q}=\ddot{q}+q^{3}=0 .
$$

Now, a proof of existence for Eq. (7.10) in the variational formulation can be based on the proof of the existence of a minimizer $q$ for the action $I(q)$ $=\int_{t_{0}}^{t_{1}} L(t, q, \dot{q}) \mathrm{d} t$. Sufficient conditions on the Lagrangian density $L$ are convexity in $\dot{q}$, i.e., the mapping $\dot{q} \mapsto L(t, q, \dot{q})$ is convex, which implies here that $L_{\dot{q} \dot{q}}(t, q, \dot{q}) \xi^{2} \geqslant 0$ for all $\xi \in \mathbb{R}$, and coercivity, i.e., $L(t, q, \dot{q}) \geqslant \alpha|\dot{q}|^{\gamma}-\beta$ for all $t, q$ and fixed $1<\gamma<\infty$ with $\alpha>0$ and $\beta \geqslant 0$, as stated in Theorem 2 on pp. 443-449 of Evans (1998), and Theorem 4.1 on p. 82 of Dacorogna (1989), for example. As is easy to observe, while the first condition-convexity-is satisfied, the coercivity is not. Hence, this variational approach does not immediately allow one to establish the existence of a solution even for this very simple example. However, from an intuitive point of view it is clear that the coercivity is satisfied once we restrict the variations in the Hamilton principle (7.9) to the open region defined by the upper energy bound (7.6). In this case we know that $\dot{q}^{2} / 2+q^{4} / 4$ $\leqslant H_{\max }$, and thus $L \geqslant|\dot{q}|^{2}-H_{\max }$, which is exactly the coercivity condition needed for proving existence. While this intuition is also supported by the fact that solutions do exist, as discussed in the following paragraph the technical details of this approach require further development.

The problem of the existence (of periodic solutions) for Hamiltonian systems was initiated by Seifert (1948) for simple Hamiltonians, i.e., $H=T(p)+V(q)$, and Weinstein (1978), who used differential geometric methods to prove existence, that is, by interpreting solutions as geodesics in a suitable Riemannian or Finsler metric. Their theory directly applies to our example. Namely, the Hamiltonian in our case is convex, as observed in the course of stability proof, so that the fixed energy $H(\mathbf{z})$ $=c$ defines a compact, convex, regular $^{20}$ surface $S$ $=H^{-1}(c)$. Thus, the conditions of the theorem in Weinstein (1978) [see also Mawhin and Willem (1989) on p. 59, Struwe (1990) on p. 58, Buttazzo et al. (1998) on p.

\footnotetext{
${ }^{20}$ Namely, $\nabla H \neq 0$ for every $\mathbf{z} \in S=H^{-1}(c)$.
} 
200] are satisfied, and therefore Hamilton's equations in our case have a (periodic) solution which orbit lies on $S$. It is important to stress that the central condition for both stability and existence is a convexity of the Hamiltonian. The variational methods in proving this result were introduced by Rabinowitz in his seminal paper (Rabinowitz, 1978), but this field still has many open problems (Ekeland, 1990).

\section{Summary}

Concluding this section, the infinite-dimensional case is still characterized by a number of technical mathematical issues, which reveal the shortcomings of current approaches to prove the (in)stability of solutions rigorously, and by the lack of clear physical interpretation and classification of dissipation-induced instabilities for partial differential equations. However, recent progress in understanding the finite-dimensional geometric picture (Krechetnikov and Marsden, 2006) provided a systematic way of looking at the infinitedimensional problems.

\section{CONCLUSIONS}

In this paper, we reviewed many-sided manifestations of the counterintuitive effect of dissipation-dissipationinduced instabilities phenomena-in both the physical and mathematical contexts. A multitude of physical applications and situations in which these types of instabilities occur indicates that this particular effect is one of the paramount ones governing instability mechanisms in nature. At the same time, a striking connection to many areas of mathematics which we tried to highlight here also indicates the fundamental importance of these phenomena. Clearly, there are many open problems and issues associated with our further understanding both at the fundamental level, e.g., infinite-dimensional systems, and on the applied side, e.g., control.

The paper contains both classical results and more recent ones, related to the deeper understanding of the geometric picture of these instabilities, some of which have never appeared in the literature. Therefore, we hope that the reader found this coherent story of dissipation-induced instability phenomena illuminating and useful.

\section{REFERENCES}

Abed, E. H., and J.-H. Fu, 1986, "Local feedback stabilization and bifurcation control, I. Hopf bifurcation," Syst. Control Lett. 7, 11-17.

Agafonov, S. A., 2002, "Stability and motion stabilization of nonconservative mechanical systems," J. Math. Sci. (N.Y.) 112, 4419-4497.

Argentina, M., and L. Mahadevan, 2005, "Fluid-flow-induced flutter of a flag," Proc. Natl. Acad. Sci. U.S.A. 102, 18291834.

Arnold, V. I., 1965, "On conditions for non-linear stability of plane stationary flows of an ideal fluid," Sov. Math. Dokl. 6,
773-777.

Arnold, V. I., 1969, "On an apriori estimate in the theory of hydrodynamic stability," Am. Math. Soc. Transl. 19, 267-269.

Arnold, V. I., 1971, "On matrices depending on parameters," Russ. Math. Surveys 26, 29-43.

Arnold, V. I., 1973, Ordinary Differential Equations (MIT, Cambridge).

Arnold, V. I., 1978, Mathematical Methods of Classical Mechanics (Springer-Verlag, New York).

Arnold, V. I., 1993, Dynamical Systems III (Springer, New York).

Arnold, V. I., and A. Avez, 1968, Ergodic Problems of Classical Mechanics (Benjamin, New York).

Berry, M. V., 1996, "The Levitron: An adiabatic trap for spins," Proc. R. Soc. London, Ser. A 452, 1207-1220.

Bloch, A., P. S. Krishnaprasad, J. E. Marsden, and T. S. Ratiu, 1994, "Dissipation induced instabilities," Ann. Inst. Henri Poincare, Anal. Non Lineaire 11, 37-90.

Bloch, A., P. S. Krishnaprasad, J. E. Marsden, and T. S. Ratiu, 1996, "The Euler-Poincare equations and double bracket dissipation," Commun. Math. Phys. 175, 1-42.

Bloch, A. M., P. Hagerty, A. G. Rojo, and M. I. Weinstein, 2004, "Gyroscopically stabilized oscillators and heat baths," J. Stat. Phys. 115, 1073-1100.

Bloch, A. M., N. Leonard, and J. E. Marsden, 1997, "Stabilization of mechanical systems using controlled Lagrangians," Proc. CDC 36, 2356-2361.

Bocquet, L., 2003, "The physics of stone skipping," Am. J. Phys. 71, 150-155.

Bolotin, V. V., 1963, Nonconservative Problems of the Theory of Elastic Stability (Macmillan, New York).

Borisenko, S. D., V. A. Goncharenko, and J. Mataracco, 2001, "On force stabilization of a dissipative system," Int. Appl. Mech. 37, 1098-1102.

Borisov, A. V., and I. S. Mamaev, 2003, "Strange attractors in rattleback dynamics," Phys. Usp. 46, 393-403.

Bracewell, R. N., and O. K. Garriot, 1958, "Rotation of artificial Earth satellites," Nature (London) 182, 760.

Buffoni, B., and M. D. Groves, 1999, “A multiplicity result for solitary-capillary waves in deep water via critical point theory," Arch. Ration. Mech. Anal. 146, 183-220.

Buttazzo, G., M. Giaquinta, and S. Hildebrandt, 1998, OneDimensional Variational Problems (Clarendon, Oxford).

Casti, A. R. R., P. J. Morrison, and E. A. Spiegel, 1998, "Negative energy modes and gravitational instability of interpenetrating fluids," Ann. N.Y. Acad. Sci. 867, 93-108.

Chang, D. E., A. M. Bloch, N. E. Leonard, J. E. Marsden, and C. A. Woolsey, 2002, "The equivalence of controlled Lagrangians and controlled Hamiltonian systems," ESAIM: Control, Optim. Calculus Var. 8, 393-422.

Cherry, T. M., 1925, "Some examples of trajectories defined by differential equations of a generalized dynamical type," Trans. Cambridge Philos. Soc. 23, 165-200.

Chetayev, N. G., 1961, The Stability of Motion (Pergamon, New York).

Clerc, M. G., and J. E. Marsden, 2001, "Dissipation-induced instabilities in an optical cavity laser: A mechanical analog near the 1:1 resonance," Phys. Rev. E 64, 067603.

Cohen, R. J., 1977, “The tippe top revisited,” Am. J. Phys. 45, 12-17.

Dacorogna, B., 1989, Direct Methods in the Calculus of Variations (Springer-Verlag, Berlin).

Daleckii, J. L., and M. G. Krein, 1974, Stability of Solutions of 
Differential Equations in Banach Space (AMS, Providence).

Dellnitz, M., I. Melbourne, and J. E. Marsden, 1992, "Generic bifurcation of Hamiltonian vector fields with symmetry," Nonlinearity 5, 979-996.

Derks, G., and T. Ratiu, 2002, "Unstable manifolds of relative equilibria in Hamiltonian systems with dissipation," Nonlinearity 15, 531-549.

Dirichlet, G. L., 1846, "Über die Stabilität des Gleichgewichts," Crelle 32, 85-88.

Earnshaw, S., 1842, "On the nature of the molecular forces which regulate the constitution of the luminiferous ether," Trans. Cambridge Philos. Soc. 7, 97-112.

Ebenfeld, S., and F. Scheck, 1995, "A new analysis of the tippe top: Asymptotic states and Liapunov stability," Ann. Phys. (N.Y.) 243, 195-217.

Ekeland, I., 1990, Convexity Methods in Hamiltonian Mechanics (Springer-Verlag, Berlin).

Evans, L. C., 1998, Partial Differential Equations (AMS, Providence, RI).

Friedlander, S., and V. Yudovich, 1999, "Instabilities in fluid motion," Not. Am. Math. Soc. 46, 1358-1367.

Galin, D. M., 1975, "Versal deformations of linear Hamiltonian systems," Am. Math. Soc. Transl. 118, 1-12.

Gantmacher, F. R., 1966, Lectures on Analytical Mechanics (Nauka, Moscow) (in Russian).

Gantmacher, F. R., 1977, The Theory of Matrices (Chelsea, New York).

Goldstein, H., 1956, Classical Mechanics (Addison-Wesley, Reading, MA).

Golubitsky, M., I. N. Stewart, and J. E. Marsden, 1987, "Generic bifurcations of Hamiltonian systems with symmetry," Physica D 24, 391-405.

Guckenheimer, J., and A. Mahalov, 1992, "Instability induced by symmetry reduction," Phys. Rev. Lett. 68, 2257-2260.

Hagerty, P., A. M. Bloch, and M. I. Weinstein, 1999, "Radiation induced instability in interconnected systems," in Proceedings of the 38th CDC (IEEE, Piscataway, NJ), pp. 651656.

Hagerty, P., A. M. Bloch, and M. I. Weinstein, 2003, "Radiation induced instability," SIAM J. Appl. Math. 64, 484-524.

Harrigan, R. M., 1983, U.S. Patent No. 4,382,245.

Herrman, G., 1967, "Stability of equilibrium of elastic systems subjected to nonconservative forces," Appl. Mech. Rev. 20, 103-108.

Holm, D. D., J. E. Marsden, T. Ratiu, and A. Weinstein, 1985, "Nonlinear stability of fluid and plasma equilibria," Phys. Rep. 123, 1-116.

Hopf, E., 1942, “Abzweigung einer periodischen Lösung von einer stationären Losüng eines Differentialsystems," Akad. Wiss. (Leipzig) 94, 3-22.

Hryniv, R. O., W. Kliem, P. Lancaster, and C. Pommer, 2000, "A precise bound for gyroscopic stabilization," Z. Angew. Math. Mech. 80, 507-516.

Jalnapurkar, S. M., and J. E. Marsden, 2000, "Stabilization of relative equilibria," IEEE Trans. Autom. Control 45, 1483 1491.

Kane, T. R., and D. A. Levinson, 1978, "A realistic solution of the symmetric top problem,” J. Appl. Mech. 45, 903-909.

Kapitsa, P. L., 1939, "Stability and transition through the critical speed of fast rotating shafts with friction," Zh. Tekh. Fiz. 9, 124-147.

Khalil, H. K., 2001, Nonlinear Systems (Prentice Hall, Englewood Cliffs, NJ).
Knobloch, E., A. Mahalov, and J. E. Marsden, 1994, "Normal forms for three-dimensional parametric instabilities in ideal hydrodynamics," Physica D 73, 49-81.

Kondrat'ev, B. P., 2000, "Dynamics and stability of resonant rings in galaxies," Astron. Rep. 44, 279-285.

Krasovskii, N. N., 1963, Stability of Motion (Stanford University Press, Stanford, CA).

Krechetnikov, R., and J. E. Marsden, 2005, "Dissipationinduced instability phenomena in infinite-dimensional systems," unpublished.

Krechetnikov, R., and J. E. Marsden, 2006, "On destabilizing effects of two fundamental non-conservative forces," Physica D 214, 25-32.

Lagrange, J. L., 1788, Mécanique Analytique (Chez La Veuve Desaint, Paris).

Langford, W. F., 2003, "Hopf meets Hamiltonian under Whitney's umbrella," in IUTAM Symposium on Nonlinear Stochastic Dynamics, edited by N. S. Namachchivaya and Y. K. Lin (Kluwer, Dordrecht), pp. 157-165.

Langthjem, M. A., and Y. Sugiyama, 2000, "Dynamic stability of columns subjected to follower loads: A survey," J. Sound Vib. 238, 809-851.

Lewis, D., 1992, "Lagrangian block diagonalization," J. Dyn. Differ. Equ. 4, 1-41.

Liaw, D.-C., and E. H. Abed, 1990, "Stabilization of thethered satellities during station keeping," IEEE Trans. Autom. Control 35, 1186-1196.

Liaw, D.-C., and E. H. Abed, 1996, "Active control of compressor stall inception: A bifurcation-theoretic approach," Automatica 32, 109-115.

Lin, C. C., 1955, The Theory of Hydrodynamic Stability (Cambridge University Press, Cambridge).

Luo, Z.-H., B.-Z. Guo, and O. Morgul, 1999, Stability and Stabilization of Infinite Dimensional Systems with Applications (Springer, London).

MacKay, R., 1991, "Movement of eigenvalues of Hamiltonian equilibria under non-Hamiltonian perturbation," Phys. Lett. A 155, 266-268.

Marsden, J. E., 1992, Lectures on Mechanics (Cambridge University Press, Cambridge).

Marsden, J. E., and J. Scheurle, 1993, "Lagrangian reduction and the double spherical pendulum," ZAMP 44, 17-43.

Marsden, J. E., J. C. Simo, D. Lewis, and T. A. Posbergh, 1989, "Block diagonalization and the energy-momentum method," Contemp. Math. 97, 297-313.

Mawhin, J., and M. Willem, 1989, Critical Point Theory and Hamiltonian Systems (Springer-Verlag, New York).

Merkin, D. R., 1974, Gyroscopic Systems (Nauka, Moscow) (in Russian).

Merkin, D. R., 1997, Introduction to the Theory of Stability (Springer-Verlag, New York).

Morrison, P. J., 1986, “A paradigm for joined Hamiltonian and dissipative systems," Physica D 18, 410-419.

Morrison, P. J., 1998, "Hamiltonian description of the ideal fluid," Rev. Mod. Phys. 70, 467-521.

Murray, C. D., 1994, "Dynamical effects of drag in the circular restricted three-body problem," Icarus 112, 465-484.

Nagata, W., and N. S. Namachchivaya, 1998, "Bifurcations in gyroscopic systems with application to rotating shafts," Proc. R. Soc. London, Ser. A 454, 543-585.

Nijmeijer, H., and van der A. Schaft, 1996, Nonlinear Dynamical Control Systems (Springer, New York). 
Nikolai, E. L., 1939, Theoretical Mechanics (GONTI, Moscow) (in Russian).

O'Brien, S., and J. L. Synge, 1953, "The instability of the tippetop explained by sliding friction," Proc. R. Ir. Acad., Sect. A 56, 23-35.

Or, A. C., 1994, “The dynamics of a tippe top,” SIAM J. Appl. Math. 54, 597-609.

Pedlosky, J., 1987, Geophysical Fluid Dynamics (SpringerVerlag, New York).

Phillips, N. A., 1951, “A simple three-dimensional model for the study of large scale extratropical flow patterns," J. Meteorol. 8, 381-394.

Pollard, H., 1966, Mathematical Introduction to Celestial Mechanics (Prentice-Hall, Englewood Cliffs, NJ).

Rabinowitz, P. H., 1978, "Periodic solutions of Hamiltonian systems," Commun. Pure Appl. Math. 31, 157-184.

Romea, R. A., 1977, "The effects of friction and $\beta$ on finiteamplitude baroclinic waves," J. Atmos. Sci. 34, 1689-1695.

Routh, E. J., 1913, Elementary Rigid Dynamics (MacMillan, London).

Rumyantsev, V. V., and S. P. Sosnitskii, 1994, "On the instability of the equilibrium of holonomic conservative systems," J. Appl. Math. Mech. 57, 1101-1122.

Seifert, H., 1948, "Periodische Bewegungen mechanischer Systeme," Math. Z. 51, 197-216.

Seyranian, A. P., and A. A. Mailybaev, 2003, Multiparameter Stability Theory with Mechanical Applications (World Scientific, Singapore).

Siegel, C. L., and J. K. Moser, 1971, Lectures on Celestial Mechanics (Springer-Verlag, New York).

Simo, J. C., D. Lewis, and J. E. Marsden, 1991, "Stability of relative equilibria. Part I: The reduced energy-momentum method," Arch. Ration. Mech. Anal. 115, 15-59.

Simon, M. D., L. O. Heflinger, and S. L. Ridgway, 1997, "Spin stabilized magnetic levitation,” Am. J. Phys. 65, 286-292.

Smale, S., 1970, “Topology and mechanics. I," Invent. Math. 10, 305-331.

Soffer, A., and M. I. Weinstein, 1999, "Resonances, radiation damping and instability in Hamiltonian wave equations," Invent. Math. 136, 9-74.
Struwe, M., 1990, Variational Methods (Springer-Verlag, Berlin).

Szebehely, V. G., 1967, Theory of Orbits: The Restricted Problem of Three Bodies (Academic, New York).

Thomson, W., and P. G. Tait, 1879, Treatise on Natural Philosophy: Part 1 (Cambridge University Press, Cambridge).

van der Meer, J.-C., 1990, "Hamiltonian Hopf bifurcation with symmetry," Nonlinearity 3, 1041-1056.

van der Shaft, A. J., 1986, "Stabilization of Hamiltonian systems," Nonlinear Anal. Theory, Methods Appl. 10, 10211035 .

Walker, J., 1979, "The mysterious "rattleback:" A stone that spins in one direction and then reverses," Sci. Am. 241 (10), 144-149.

Wang, L.-S., and P. S. Krishnaprasad, 1992, "Gyroscopic control and stabilization," J. Nonlinear Sci. 2, 367-415.

Wang, L.-S., P. S. Krishnaprasad, and J. H. Maddocks, 1991, "Hamiltonian dynamics of a rigid body in a central gravitational field," Celest. Mech. Dyn. Astron. 50, 349-386.

Weinstein, A., 1978, "Periodic orbits for convex Hamiltonian systems," Ann. Math. 108, 507-518.

Whitney, H., 1943, "The general type of singularity of a set of $2 n-1$ smooth functions of $n$ variables," Duke Math. J. 10, $161-172$.

Whittaker, E. T., 1917, A Treatise on the Analytical Dynamics of Particles and Rigid Bodies (Cambridge University Press, Cambridge).

Wiggins, S., 2003, Introduction to Applied Nonlinear Dynamical Systems and Chaos (Springer, New York).

Wintner, A., 1947, The Analytical Foundations of Celestial Mechanics (Princeton University Press, Princeton).

Yudovich, V. I., 1989, The Linearization Method in Hydrodynamic Stability Theory (AMS, Providence).

Zajac, E. E., 1964, "The Kelvin-Tait-Chetaev theorem and extensions," J. Astronaut. Sci. 11, 46-49.

Ziegler, H., 1952, "Die Stabilitätskriterien der Elastomechanik," Ing.-Arch. 20, 49-56.

Ziegler, H., 1953, "Linear elastic stability,” Z. Angew. Math. Phys. 4, 89-121. 